\title{
Fitting the integrated Spectral Energy Distributions of Galaxies
}

\author{
Jakob Walcher • Brent Groves • Tamás Budavári • \\ Daniel Dale
}

\section{Contents}

1 Introduction

2 Modelling galaxy SEDs

2.1 Stars ..............................

2.1.1 Simple stellar populations . . . . 3

2.1.2 Validation of SSP predictions ... 5

2.1.3 Current Issues with SSPs . . . . . 6

2.2 The ISM around the stars . . . . . . . . . 7

2.2.1 Interstellar gas . . . . . . . . 7

2.2.2 Interstellar dust . . . . . . . 8

2.2.3 Combining stellar and dust emission 11

2.3 Evolution of Galaxies . . . . . . . . . . . 13

3 Constructing observed galaxy SEDs 15

3.1 Spectral response curve and resolution ... 15

3.2 Spatial resolution, aperture bias and matching 15

3.3 Examples of multi-wavelength datasets . . 16

3.3.1 The Spitzer Local Volume Legacy spatially resolved SEDs _... . . 16

3.3.2 The Herschel ATLAS - unresolved SEDs ............ 16

3.3.3 The SWIRE templates . . . . . . 17

3.3.4 Further examples . . . . . . . . 17

Jakob Walcher

Research and Scientific Support Department, European Space Agency, Keplerlaan 1, 2200AG Noordwijk, The Netherlands

Brent Groves

Sterrewacht Leiden, Leiden University, P.O. Box 9513, 2300 RA Leiden, The Netherlands

Tamás Budavári

Dept. of Physics and Astronomy, The Johns Hopkins University, $3400 \mathrm{~N}$. Charles Street, Baltimore, MD 21218, USA

Daniel Dale

Department of Physics and Astronomy, University of Wyoming, Laramie, WY 82071, USA
4 Methods and validation of SED fitting 18

4.1 Parametrizing SED models . . . . . . . . 18

4.2 Spectral indices . . . . . . . . . . . 19

4.3 Principal Component Analysis . . . . . 20

4.4 Spectral fitting by inversion . . . . . . . 21

4.4.1 Method ............ 21

4.4.2 Non-linear inversion codes . . . . 22

4.4.3 Non-linear physics . . . . . . . 22

4.4.4 Validation . . . . . . . . . 22

4.5 Bayesian inference . . . . . . . . . 23

4.5.1 Method ........... 23

4.5.2 Libraries and priors . . . . . . . 24

4.5.3 Validation ........... 25

4.6 Method-independent caveats . . . . . . 28

5 Results of SED Fitting: Photometric redshifts 29

5.1 Methods . . . . . . . . . . . . . 29

5.1.1 Empirical techniques . . . . . . 29

5.1.2 Template Fitting . . . . . . . 31

5.2 Calibration and error budgets . . . . . . 31

5.2.1 Template accuracy . . . . . . . 32

5.2.2 Spectroscopic Calibration of Photo-zs 32

5.2.3 Signal-to-noise Effects . . . . . . 33

5.3 A unified framework . . . . . . . . . 33

5.4 The State of Photometric Redshifts . . . . 33

6 Results of SED Fitting: Physical Properties 34

6.1 Stars . . . . . . . . . . . . . 34

6.1.1 Stellar masses . . . . . . . . . . 34

6.1.2 Deriving SFHs from spectroscopy . 35

6.1.3 Identifying and studying outliers . . 36

6.2 Dust . . . . . . . . . . . . . 37

6.2.1 Attenuation by Dust . . . . . . 37

6.2.2 Dust Emission . . . . . . . . . 37

6.2.3 Dust in the UV to IR . . . . . . . 39

6.2.4 Star Formation Rate from the IR . . 39

6.3 Fitting the full UV to FIR SED . . . . . . 40

7 Conclusions 
Abstract Fitting the spectral energy distributions (SEDs) of galaxies is an almost universally used technique that has matured significantly in the last decade. Model predictions and fitting procedures have improved significantly over this time, attempting to keep up with the vastly increased volume and quality of available data. We review here the field of SED fitting, describing the modelling of ultraviolet to infrared galaxy SEDs, the creation of multiwavelength data sets, and the methods used to fit model SEDs to observed galaxy data sets. We touch upon the achievements and challenges in the major ingredients of SED fitting, with a special emphasis on describing the interplay between the quality of the available data, the quality of the available models, and the best fitting technique to use in order to obtain a realistic measurement as well as realistic uncertainties. We conclude that SED fitting can be used effectively to derive a range of physical properties of galaxies, such as redshift, stellar masses, star formation rates, dust masses, and metallicities, with care taken not to over-interpret the available data. Yet there still exist many issues such as estimating the age of the oldest stars in a galaxy, finer details of dust properties and dust-star geometry, and the influences of poorly understood, luminous stellar types and phases. The challenge for the coming years will be to improve both the models and the observational data sets to resolve these uncertainties. The present review will be made available on an interactive, moderated web page (sedfitting.org), where the community can access and change the text. The intention is to expand the text and keep it up to date over the coming years.

Keywords methods: data analysis, radiation mechanisms: general, techniques: photometric, techniques: spectroscopic, galaxies: ISM, galaxies: stellar content

\section{Introduction}

Integrated spectral energy distributions (SEDs) are our primary source of information about the properties of unresolved galaxies. Indeed, the different physical processes occurring in galaxies all leave their imprint on the global and detailed shape of the spectrum, each dominating at different wavelengths. Detailed analysis of the SED of a galaxy should therefore, in principle, allow us to fully understand the properties of that galaxy. SED fitting is thus the attempt to analyze a galaxy SED and to derive one or several physical properties simultaneously from fitting models to an observed SED. This is in contrast to searching a single feature that could constrain a single parameter (a prominent example would be the use of the $\mathrm{H} \alpha$ line to derive the star formation rate, SFR).

The aim of this review is to present the state of the art in the area of fitting the ultraviolet (UV) to far infrared (FIR)
SEDs of galaxies. It grew from and presents a summary of a workshop held at the Lorentz center in Leiden in November 2008. As the field is extremely large we attempt here a somewhat novel approach to the process of writing a review. We consider this text as a starting point. The text will then be made available at www.sedfitting.org and we invite the community to sent us corrections and additions. We particularly hope that this will help in covering the work that we might have missed. We also made no attempt (yet) to cover the new results of the last year, in particular from Herschel.

Progress in many areas that affect SED fitting has been made recently. A major development in the last decade has been the advent of new observing facilities and large surveys at all wavelengths of the spectrum, enabling astronomers for the first time to observe the full SEDs of galaxies at wavelengths from the $\mathrm{X}$-rays to the radio. The same surveys have also pushed the distance of the farthest galaxy whose SED is amenable to study to redshifts higher than 6 . At the same time, tools and models have been created that aim to extract the complex information imprinted in the SEDs. Also, useful semi-analytic models of galaxy formation have appeared that provide realistic predictions for the properties of galaxies as they would be formed in the current cosmological standard model. Not only are astronomers exploiting the available data, but the next generation of surveys is now in the planning phase.

For this review we concentrate on observations from the ultraviolet to the far infrared, including both multi-band photometric and spectral data. We thus treat the light emitted by stars, either directly or processed by the gas and dust of the surrounding interstellar medium. At wavelengths outside the regime considered here, such as the $\mathrm{X}$-ray and radio wavelengths, non-stellar processes (or at least those not directly associated with stellar light) such as shocks, accretion onto compact objects, etc. dominate. While many of these can be associated with the star formation history of a galaxy (e.g. supernova rate and recent star formation), these processes require a higher order of complexity generally not considered in most current models of galaxy spectra, and hence we do not discuss these wavelengths in the rest of this review. We also do not treat the contribution of active galactic nuclei to the SEDs of galaxies.

Our initial aim of both the workshop and this review was to set up a basic framework to answer the main question relating to SED fitting: considering the difficulties with the models, considering the limitations of the data and considering the fitting technique, what is the true uncertainty and limitations on the properties that can be determined from galaxy SED fitting?

This review is structured as follows: in Section 2 we review the basics of galaxy ultraviolet to infrared SED modelling, from galaxy formation to the production of and processing of the radiation from these galaxies. We especially 
mention some of the current issues and main uncertainties of the modelling of galaxy SEDs. In Section 3 we provide a short overview of the intricacies of assembling multiwavelength SEDs. In Section 4 we present techniques and algorithms for SED fitting, and - most importantly - efforts at validating the results from the SED fitting procedure with independent data. Section 5 presents a review of photometric redshift determinations, a special case of a physical property derived from SED fitting, as it can be compared to and calibrated on independently determined data, spectroscopic redshifts. Finally, Section 6 showcases some recent results from application of the SED fitting procedures, where we hope to emphasize the variety of problems to which SED fitting can significantly contribute.

\section{Modelling galaxy SEDs}

Galaxies emit across the electromagnetic spectrum. Excluding those galaxies dominated by an accreting supermassive black hole at their nucleus (AGN), the ultraviolet to infrared spectra of all galaxies arises from stellar light, either directly or reprocessed by the gas and dust of the surrounding interstellar medium (ISM). Thus the UV-to-IR spectral energy distribution or SED contains a large amount of information about the stars of a galaxy, such as the stellar mass to light ratio, and the surrounding ISM, such as the total dust mass. However, to extract such information, models are necessary in order to connect physical properties of the galaxy with the observed SED. In this section we discuss such models, beginning with the stellar spectrophotometric models, moving on to the transfer of the radiation of these stars in a galaxy through the ISM, and finally how to connect these with the larger picture of galaxy formation and evolution. We use the following abbreviations for designing wavelength ranges, though the exact boundaries between wavelength regimes are not sharp: ultraviolet (UV) for $\lambda<3500 \AA$, optical for $3500<\lambda<8000 \AA$, near infrared (NIR) $0.8<\lambda<3 \mu \mathrm{m}$, midinfrared (MIR) $3<\lambda<25 \mu \mathrm{m}$, far-infrared (FIR) $25<\lambda<250$ $\mu \mathrm{m}$, sub-mm $0.25<\lambda<1 \mathrm{~mm}$, and radio $\lambda>1 \mathrm{~mm}$.

\subsection{Stars}

In its simplest sense, a galaxy is a population of stars ranging from numerous, low-luminosity, low-mass stars, to the bright, short-lived, massive OB stars. On closer examination, these stars are distributed in both metallicity content and age ranging from when the galaxy first formed to those newly born. The method of creating a galactic spectrum through the sum of the spectra of its stars is called stellar population synthesis and was pioneered in works by Tinsley (1972), Searle et al. (1973) and Larson and Tinsley (1978). A simplification for the modelling of galactic SEDs is that the emitted light can be represented through a sum of spectra of simple stellar populations (SSPs) with different age and element abundances. Here a SSP is an idealized singleage, single-abundance ensemble of stars whose distribution in mass depends on both the initial distribution and the assumed age of the ensemble.

There are two main methods used by current stellar spectrophotometric models to compute the SEDs of SSPs: The first is called 'isochrone synthesis'. It uses the locus of stars with the same age, called an isochrone, in the HertzsprungRussel diagram and then integrates the spectra of all stars along the isochrone to compute the total flux. This method was established by Chiosi et al. (1988); Maeder and Meynet (1988) and in particular Charlot and Bruzual (1991) and is currently used by the majority of stellar population models. The second uses the 'fuel consumption' approach. One of the problems of the isochrone synthesis method was that isochrones are calculated in discrete steps in time and therefore phases where stellar evolution is more rapid than theses timesteps were not well represented (the most famous example of the last years being the thermally pulsing asymptotic giant branch stars). Models using the fuel consumption theorem circumvent this problem by changing the integration variable above the main sequence turnoff to the stellar fuel, i.e. the amount of hydrogen and helium used in nuclear burning. The fuel is integrated along the evolutionary track. The main idea is that the luminosity of the post-main sequence stars, which are the most luminous, is directly linked to the fuel available to stars at the turnoff mass (for full details, see e.g. Buzzoni 1989; Maraston 1998, 2005). While these methods are fundamentally different in their integration methods, most of the issues discussed here in terms of stellar evolution and stellar libraries apply to both.

\subsubsection{Simple stellar populations}

The spectrum (flux emitted per unit frequency per unit mass), $L_{v}$, of a SSP of mass M, age $t$, and metallicity $Z$ is given by the sum of the individual stars:

$L_{v}(t, Z)=\int_{\mathrm{M}} \phi(\mathrm{M})_{t, Z} L_{v}(\mathrm{M}, t, Z)$.

In practice the emitted light is dominated by the most massive, luminous stars.

The stellar mass function, $\phi(\mathrm{M})_{\mathrm{t}, \mathrm{Z}}$, is computed from an initial mass function (IMF, $\phi_{0}(\mathrm{M})$ ) and stellar evolution, which describes when and which stars will stop contributing to the SSP spectra because they end their lives either as Supernovae or as white dwarfs. The IMF describes the distribution in mass of a putative zero-age main sequence stellar population and is an input parameter of stellar population synthesis models. The IMF is usually limited between a minimum and maximum stellar mass (generally 
$\left.\mathrm{M}_{\min } \sim 0.05-1.0 \mathrm{M}_{\odot} ; \mathrm{M}_{\max } \sim 100-150 \mathrm{M}_{\odot}\right)$. Three empirical forms are most commonly used: a simple power-law model (Salpeter 1955; Massey 1998), a broken power-law (Kroupa 2001), or a lognormal form (Chabrier 2001). However whether these forms hold in all conditions and for all redshifts is still an open question (A good coverage of this field can be found in the "IMF@50" proceedings, E. Corbelli, F. Palla, \& H. Zinnecker 2005).

The true difficulty of calculating equation 1 lies in the second part, determining the SED $\left(L_{v}\right)$ of a star of initial mass, $\mathrm{M}$, age, $t$, and metallicity, $Z$. This requires; 1$)$ the computation of stellar evolutionary tracks that determine where a star of given stellar parameters (e.g. mass M, age $t$ and abundance $Z$ ) lies on the Hertzsprung-Russel diagram or $\log g-\mathrm{T}_{\text {eff }}$ diagram, to build up the stellar 'isochrone', and 2) the computation or empirical building of a stellar library of $L_{v}$ with full coverage of $\log g, \mathrm{~T}_{\text {eff }}$, and $Z$ to determine what the resulting spectrum of such a star is.

The creation of stellar isochrones requires a large grid of evolutionary tracks, created by modelling the evolution of stars of a given initial mass and metal content. Over the past few decades, much work has gone into providing homogeneous sets of stellar tracks from different groups, e.g. Padova (Marigo and Girardi 2007, Marigo et al. 2008), Geneva (Lejeune and Schaerer 2001), Yale (Demarque et al. 2004), MPA(Weiss and Schlattl 2008), BaSTI (Pietrinferni et al. 2009). For SSP modelling, the models generally run from the start of the main sequence (Zero Age Main Sequence, ZAMS) to some end point of the star, such as a supernova or the asymptotic giant branch (AGB) phase. Originally computed only for a solar metallicity composition and a few stellar masses, sets of homogeneous stellar evolutionary tracks now exist for a wide range of initial masses (from $\sim 0.1 \mathrm{M}_{\odot}$ to $\sim 120 \mathrm{M}_{\odot}$; see e.g. Girardi et al.|2000, Meynet and Maeder 2005) and metallicities $\left(\sim 0.01\right.$ to $\left.\sim 4 \mathrm{Z}_{\odot}\right)$. In most stellar evolutionary modelling it has been assumed that for all stellar masses the elemental composition is the same for a given metallicity, however more recently the evolutionary effects of elemental variations such as $\alpha$-enhancement (e.g. Salasnich et al. 2000) or individual element variations (e.g. Dotter et al. 2007) have been investigated. However problems still remain in the field, with the different treatments by the different groups still giving distinct evolutionary tracks even with the same inputs, as shown in figure 1.

While the evolutionary tracks lead to the generation of an isochrone, to determine a SSP spectrum, a library of stellar spectra is needed, covering the necessary parameter space in $\log \mathrm{T}_{\text {eff }}, \log g$, metallicity etc. As with the evolutionary tracks, stellar libraries have improved significantly in recent years, with both fully theoretical (e.g. Kurucz 1992, Westera et al. 2002; Smith et al. 2002: Coelho et al.2005; Martins et al.2005: Lançon et al. 2007) and empirical, e.g. STELIB (Le Borgne et al. 2003), MILES (Sánchez-Blázquez et al.
2006; Cenarro et al. 2007), Indo-US (Valdes et al. 2004), ELODIE (Prugniel and Soubiran|2001; Prugniel et al. 2007), HST/NGSL (Gregg et al. 2004) libraries covering much greater parameter spaces and increasing in both spectral and parameter resolution (see figure 2). Unlike the optical, the UV still suffers from incomplete libraries which is specially important for fully exploiting data on high redshift galaxies (see e.g. Pellerin and Finkelstein 2009). The question about which of empirical or theoretical libraries is preferable is a matter of the specific application (for a short review on both sets of libraries and their respective issues, see Coelho 2009). The main benefit of the empirical libraries is that they are based on real stars and thus avoid uncertainties in stellar atmosphere structure or in opacities. On the other hand, due to the observational limits they cover a restricted parameter space biased towards Milky way compositions (see e.g. Cenarro et al. 2007). Additionally, the determination of their fundamental parameters can be difficult for some types of stars and is itself based on stellar models. Conversely theoretical libraries can cover a much larger parameter space and at any chosen resolution (see e.g. Martins et al. 2005). There are still known problems in the comparison between the observed and theoretical stellar spectra (Martins and Coelho 2007). Two specific examples for problems of theoretical models are incomplete line lists (Kurucz 2005), problematic particularly at high spectral resolution, and the modelling of the IR emission (Lançon et al. 2007), which is particularly difficult for stars in the luminosity classes I and II (A. Lançon, talk at workshop). The way forward may be a synthesizing approach, as suggest by Walcher et al. (2009), aimed at using the strengths of both kinds of libraries. As with the evolutionary tracks, most libraries are limited to single compositions for a given metallicity. However recently this also has been changing, with stellar libraries exploring abundance changes such as $\alpha$-enhancement as well (e.g. Coelho et al. 2007) .

It is not much of an overstatement to say that the magic of stellar population evolutionary synthesis spectrophotometric codes lies in interpolation. Indeed, to go from evolutionary tracks to isochrones (quoting Maeder and Meynet 1988) "the interpolation between evolutionary tracks must be properly based on point of corresponding evolutionary status" (see also Prather 1976), and similarly to go from an isochrone to SSP SED, the correct, often interpolated, spectrum must be found for each mass bin. These stellar population synthesis (SPS) codes, using stellar evolutionary tracks and stellar libraries, then calculate Equation 11. Besides interpolation, the challenge is to create the most homogeneous and most accurate set of input ingredients, interpolating in an appropriate manner when necessary in dependence on the coverage and strengths of these sets. Stellar population models predicting full spectra include; Fioc and RoccaVolmerange (1997, PEGASE), Bressan et al. (1998, used 


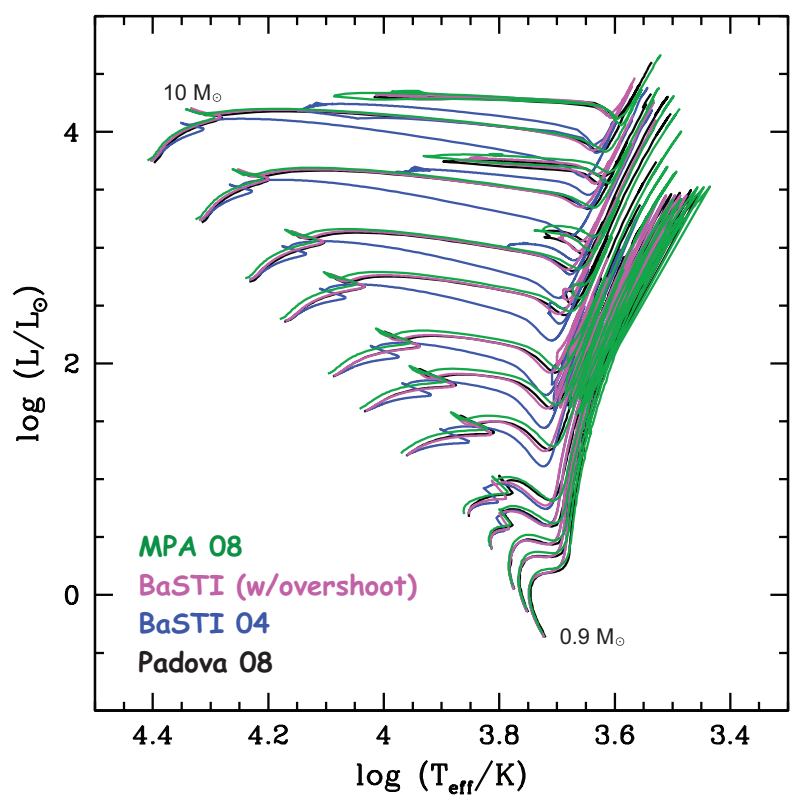

Fig. 1 Evolutionary tracks of solar composition low mass stars (0.9 - $10 \mathrm{M}_{\odot}$ ) demonstrating the differences between four different models (as labelled): MPA08 (Weiss and Schlattl 2008), BaSTI04 (with/without overshoot; Pietrinferni et al. 2009), and Padova08 (Marigo and Girardi 2007, Marigo et al.2008) [Courtesy S. Charlot].

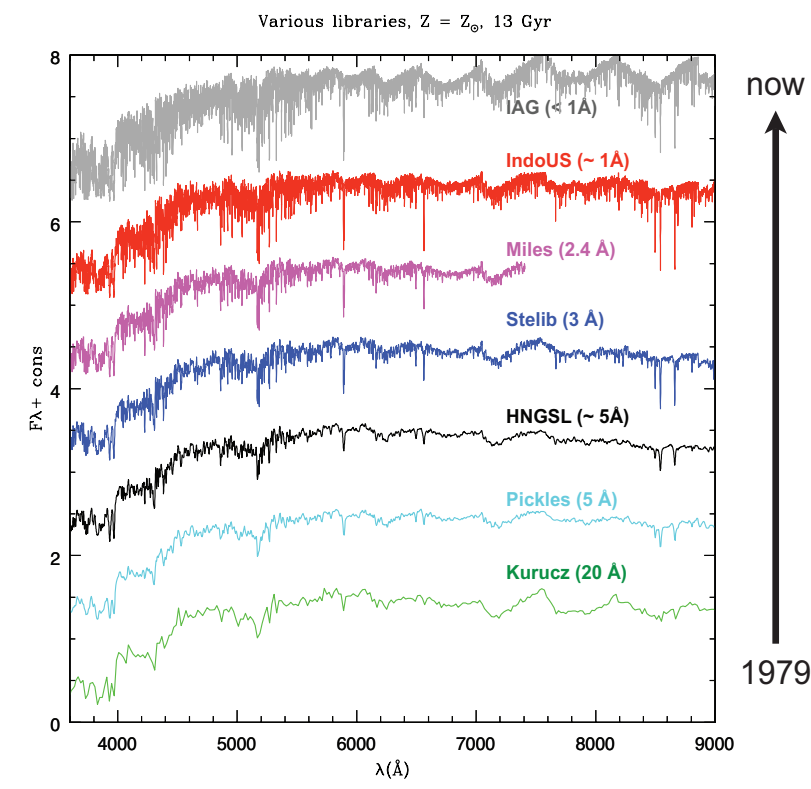

Fig. 2 Optical spectra from both theoretical and empirical stellar libraries (as labelled) demonstrating the improvement of spectral resolution over time with the associated improvement in library size [Courtesy S. Charlot]. in GRASIL) and Leitherer et al. (1999) and Vázquez et al. (2007) (both Starburst99), Vazdekis (1999), Schulz et al. (2002), Cerviño et al. (2002), Robert et al. (2003), Bruzual and Charlot (2003, GALAXEV, also commonly referred to as BC03), Le Borgne et al. (2004, PEGASE-HR), Maraston (2005, M05, based on fuel consumption theorem), Lançon et al. (2008), Mollá et al. (2009). Currently less frequently used are fully theoretical stellar population models such as González Delgado et al. (2005); Coelho et al. (2007). However, as recently shown by Walcher et al. (2009), a combination of semi-empirical and fully theoretical models holds great promise for the future. As a result of the improvements in stellar evolutionary tracks and stellar libraries, as well as the codes themselves, stellar population synthesis models today can recreate broad-band UV to NIR SEDs and high-resolution spectra in the optical remarkably well.

\subsubsection{Validation of SSP predictions}

As SSP spectra form the basis of all fitting of galaxy SEDs and as the complexities of real galaxies may introduce degeneracies and further uncertainties in the resulting interpretations, it is of primary importance to validate directly the predictions of the stellar population synthesis models for SSPs (see e.g. Bruzual A. 2001). For the impact of uncertainties in the stellar parameters effective temperature, surface gravity, and iron abundance on the final SSPs see Percival and Salaris (2009).

The ideal testbed for such validations are simple stellar populations occurring in nature, i.e. co-eval stellar populations such as globular clusters (GCs), open clusters, and young star clusters. These objects have been used as such for some time (e.g. Renzini and Fusi Pecci 1988, González Delgado and Cid Fernandes 2010), but unfortunately, the exact equivalence between star clusters and SSPs breaks down for a number of reasons:

1) In star clusters, the stellar populations are affected by the dynamical evolution of the cluster, which leads to mass segregation, and evaporation of low-mass stars in GCs and, in young star clusters, the expulsion of gas early in their life time may lead to dissolution (open clusters) or to the loss of a significant number of stars (though see Anders et al.2009. for recent work on dealing with this in SSP models).

2) Clusters also contain exotic stars (e.g. blue straggler stars, discussed in the following section) which influence the integrated light of the cluster but are not accounted for by most population synthesis models (though see Xin et al. 2007, for a discussion on how to account for these).

3) Finally, star clusters only have a finite number of stars. In a less than $10^{5} \mathrm{M}_{\odot}$ star cluster, the number of bright stars is so small, that stochastic fluctuations in the photometric properties of the cluster are common (Barbaro and Bertelli 1977; Lançon and Mouhcine 2000a, Cerviño and Luridiana 2004, 2006, Piskunov et al. 2009). 
In the study of individual clusters, most of these problems might be alleviated by concentrating on the most massive specimens (W3, $\omega$ Cen, starburst clusters), but these have the tendency to exhibit multiple rather than single (simple) stellar populations (e.g. Lee et al. 1999). Thus, one needs to study star cluster populations for comparison with SSP models and to account for the influence of the stochastic fluctuations on the color-luminosity distribution. Looking the other way around, within a given error box for the observed colors, a complex distribution of possible ages is possible (Fouesneau et al., talk at workshop). While multi-wavelength observations help, they do not completely eliminate the problem.

\subsubsection{Current Issues with SSPS}

Even though significant improvements in the evolutionary tracks and stellar libraries have been made in the last decade, significant challenges remain, as some parts of stellar evolution are only weakly understood, and hence poorly treated. The most important of these tend to be short lived but bright phases: massive stars, thermally pulsing asymptotic giant branch (TP-AGB) stars, extreme horizontal branch stars (EHB) and blue stragglers.

Massive stars, due to their rapid evolution and short lifetime, prove to be difficult to model and observe at all phases. Additional difficulties arise in that they tend to be buried by the interstellar material that they formed from for a large fraction of their lifetime, and experience high stellar winds (and hence strong mass evolution over their lifetime). Yet massive stars are a vital component of SSP modelling because they are so luminous and can thus dominate a SSP spectrum, and because they give rise to most of the ionizing flux and resulting nebular emission-line contribution. Previous modelling of massive star evolution paid particular attention to the size of the convective core and stellar mass loss, yet recent theory has indicated the significant, if not dominant, role that stellar rotation has on the evolution of these stars (Meynet and Maeder 2005). Vázquez et al. (2007) show in their recent stellar population model that, as rotating stars tend to be bluer and more luminous than in earlier models, even the ionizing spectrum can be significantly altered. These differences have consequences when interpreting the SEDs of young galaxies, such as decreasing the determined mass or star formation rates.

TP-AGB stars are short-lived, cool but luminous components of evolved stellar populations that tend to be more prominent at low metallicities. Due to the short lifetime of this stellar phase, as well as the inherent instability of the pulsations, such stars are difficult to model. Yet due to their relatively high luminosities (see figure 3) they can significantly alter the mass-to-light $\left(\mathrm{M}_{*} / \mathrm{L}\right)$ ratio of intermediateage populations and it is thus important to properly include them. Previously, several different theoretical and semiempirical recipes had been used in stellar population synthesis models leading to large discrepancies between SSP spectra (Vassiliadis and Wood 1993; Maraston 1998). Attention has been focused on these stars since Maraston (2005) raised this issue, leading to rapid progress in the modelling (Marigo and Girardi 2007), largely reducing these differences in the broad-band photometry.

The emission of SSPs near $10 \mathrm{Myr}$ is dominated by luminous red supergiants, showing that more problems exist in the NIR than only TP-AGB stars. At higher spectral resolution in the NIR, comparisons of SSPs based on different libraries of synthetic stellar spectra and different isochrones show large residuals in the whole range from 1 to $2.5 \mu \mathrm{m}$ and in particular for young to intermediate ages (A. Lançon, talk at workshop). Additionally, ages derived from NIR and optical spectroscopy are discrepant by factors of two in this age regime. Resolving these problems will surely lead to improved predictions of stellar population models at all wavelengths.

EHB stars represent the most luminous hot component in old stellar populations. Understanding these stars and implementing them in SSP codes is important because they could be mistaken for low-level star formation in more evolved, early-type galaxies. Unfortunately, the evolution of EHB stars is not fully understood. While HB morphology may be dependent upon metallicity, some metal-rich stellar populations show HB stars bluer than expected (Heber 2008). The second-parameter problem with the morphology of the horizontal branch (for a review see Catelan 2009) will need to be solved before significant progress can be expected in this field. Meanwhile, the comparison between model SSP spectra and the data at old ages is affected by these uncertainties (Ocvirk|2010).

Blue straggler stars are, as the name suggests, stars that extend beyond the main sequence turn-off. Their origin is still unknown, though it is believed to be associated with binary star evolution, either through mass transfer or merging (Tian et al. 2006; Ferraro et al. 2006). As with EHB stars, blue stragglers can affect the interpretation of earlytype spectra giving younger average ages. These stars point towards a fundamental limitation of current SSP modelling, in that effects of binary evolution are not included. While this causes little difference in most cases, in some situations (such as where blue stragglers may dominate) recent binary stellar population models may be more suitable (Han et al. 2007).

The reader is also referred to the series of papers Conroy et al. (2009); Conroy and Gunn (2010b) for a recent systematic study of some of the uncertainties affecting SSP models.

Yet, even with all these remaining issues, SSP modelling has advanced significantly in recent years, with simple Charlot \& Bruzual (2010, in preparation) exponentially-declining 


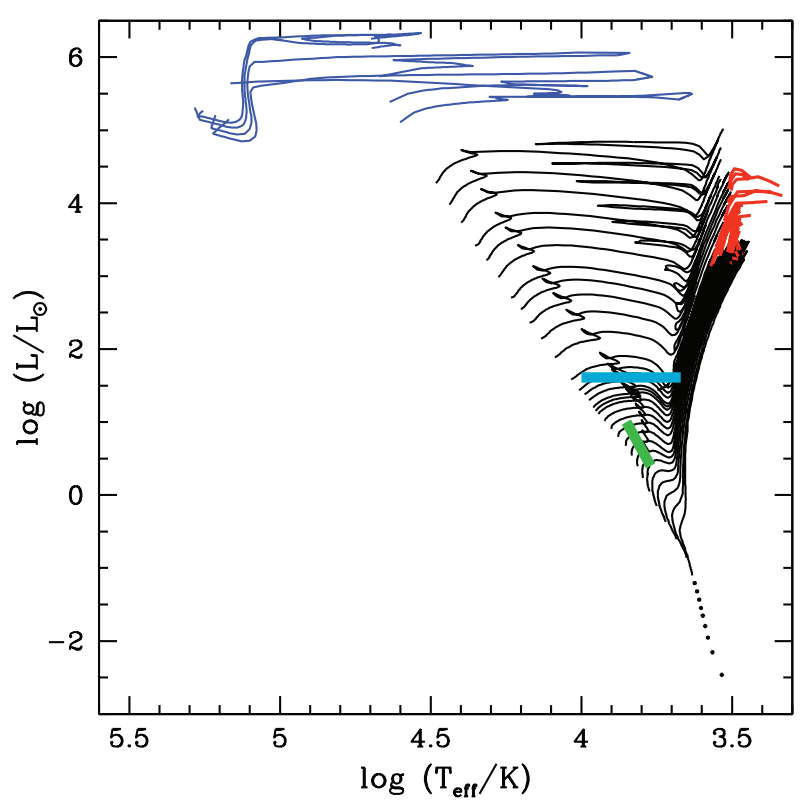

Fig. 3 Effective stellar temperature versus luminosity diagram showing stellar evolutionary tracks, with the problem/uncertain areas marked. As a basis, the Padova (Marigo et al. 2008) tracks for 0.1 to $15 \mathrm{M}_{\odot}$ are shown in black. At higher luminosities (blue curves) are evolutionary tracks for the rapidly evolving high mass $\left(\sim 100 \mathrm{M}_{\odot}\right)$ stars. The red curves show the new evolutionary tracks for the thermally pulsing asymptotic giant branch (TP-AGB) phase, while the blue and green rectangles show the regions dominated by extreme horizontal branch (EHB) and blue straggler stars respectively. [Courtesy S. Charlot]
SFH + burst models able to reproduce 1000's of optical SDSS spectra (of $\sim 5 \AA$ resolution) to a few percent (see Section 4.4.4.

\subsection{The ISM around the stars}

SSP models are state of the art for producing the spectra of stellar populations, yet they are not sufficient alone for reproducing the spectra of galaxies. Stars are the dominant power sources within galaxies (excluding AGN). However, the radiation from stars is absorbed and processed by the gas and dust that lies between the stars, the interstellar medium (ISM). This absorption must be accounted for when comparing SSP models with optical/UV observations and a treatment of the radiative transfer of the stellar light through the ISM and subsequent ISM emission is necessary if the full UV-IR SED is to be understood.

While the gas and dust are in reality intermingled within the ISM, in practice they are often treated as separate components because their absorption properties have a different wavelength dependence.

\subsubsection{Interstellar gas}

Interstellar gas is predominantly treated as atomic in the modelling of galaxies. While molecular gas is clearly present in many galaxies, it has generally a low volume filling factor, meaning that rarely contributes significantly to the overall opacity in a galaxy. It is only a noticeable opacity source in specific spectral features or in galaxies dominated by nuclear/heavily obscured sources, such as AGN and ultra-luminous IR galaxies (ULIRGs). Molecular gas emission in galaxies is predominantly seen at longer wavelengths (NIR and longer) and is generally treated to arise mostly from "Photodissociation Regions", where the gas is heated by the diffuse interstellar radiation field of the galaxy. Although it provides insight into the molecular phase of the ISM, molecular emission is not considered to be a significant contributor to the overall SED of a galaxy (for further details see reviews by e.g. Young and Scoville 1991; Hollenbach and Tielens (1997).

Atomic gas however is the dominant opacity source in the extreme-UV $(\gtrsim 13.6 \mathrm{eV})$. It reprocesses this light into strong emission lines in the UV, optical and IR. It is thus especially important for young, actively star-forming galaxies. Usually, it is assumed that all hydrogen ionizing photons $(h v>13.6 \mathrm{eV})$ are absorbed locally, within a small volume around the ionizing sources (approximately the Strömgren sphere ${ }^{1}$, which is appropriate due to the high opacity in this

${ }^{1}$ The Strömgren sphere is defined as; $\mathrm{V}_{s}=Q\left(\mathrm{H}^{0}\right) /\left(n_{\mathrm{H}}^{2} \alpha_{B}\right)$, where $Q\left(\mathrm{H}^{0}\right)$ is the total number of ionizing photons, $n_{\mathrm{H}}$ is the hydrogen number density, and $\alpha_{B}$ is the case $B$ hydrogen recombination rate. 
wavelength regime. This energy is then re-emitted in the hydrogen recombination lines, which correlate directly with the total number of ionizing photons (see e.g. Storey and Hummer 1995, Leitherer et al. 1999). However to determine the emission from other elements, or to take account of both gas and dust absorption, full radiative transfer is needed using photoionization codes such as CLOUDY (Ferland et al. 1998) or Mappings III (Groves et al.|2008). For further details, see reviews by Ferland (2003) and Stasińska (2007).

As both the number density and absorption cross-section of dust is low relative to hydrogen in the EUV, dust is often ignored as an opacity source. However, as hydrogen absorption is limited by the recombination rate, dust absorption becomes relatively more important as the strength of the ionizing radiation field increases, becoming the dominant EUVopacity source when $q\left(\mathrm{H}^{0}\right)>\alpha_{B} / \kappa \sim 5 \times 10^{8} \mathrm{~cm} \mathrm{~s}^{-1}$ assuming typical values for the dust opacity, $\kappa$ (Dopita et al.|2002). This value of the ionization parameter $q$ is well above the average value measured for star-forming galaxies (see e.g. Kewley et al. 2001, meaning negligible EUV absorption by dust in typical $\mathrm{H}$ II regions $(<5 \%)$, but such high values may be reached within compact $\mathrm{H}$ II regions and AGN meaning dust will absorb a significant fraction of EUV photons (Dopita et al. 2002; Draine 2010).

\subsubsection{Interstellar dust}

Interstellar dust has been a field of constant inquiry since it was first realized that an obscuring material existed between the stars and a large body of research exists on the composition, shape and distribution of dust exists (see Draine 2003. for a detailed review of the field, and some remaining questions about dust).

Most of our understanding of interstellar dust has come locally, from observations within our own Galaxy and the Magellanic clouds, and also through theoretical and experimental laboratory work. It is generally accepted that the grains can be considered to be composed of three different compositions; graphitic/amorphous carbon grains, amorphous silicate grains, and polycyclic aromatic hydrocarbons (PAHs), which may or may not be an extension of the carbonaceous grains. The former two were found to reproduce the observed extinction along different lines-of-sight within our galaxy (Mathis et al. 1977), while the latter were added to explain unidentified emission bands in the mid-IR (Leger and Puget 1984). Other forms of dust have been suggested, such as SiC (Treffers and Cohen 1974), and ice is expected to form on grains in the coldest environments such as deep in molecular clouds, but generally only these forms are considered in the SED modelling of galaxies.

The size distribution of interstellar dust grains is thought to be power-law in nature, with a distribution $\mathrm{N}(a) \propto a^{-3.5}$ or similar, with the average cross-section dominated by small grains, but mass dominated by large. This slope arises from both theory (Jones et al. 1996) and matching observations (Mathis et al. 1977; Draine and Lee 1984, Weingartner and Draine 2001).

To obtain the optical data used for dust calculations in SED modelling, the size distribution and types are then convolved with absorption/emission cross-sections and scattering cross-sections and phase functions which are determined by both laboratory observations and Mie theory (see Draine and Li 2007a, Zubko et al. 2004, and references within). PAHs are treated slightly differently as their composition is not fully understood, and their properties can change significantly with the charge of the grains, and thus have more empirical based treatments (Weingartner and Draine 2001, see e.g.). Altogether these form the dust models which are used most often in SED modelling, such as Draine and Li (2007a) or Zubko et al. (2004), that have been successfully compared with determined depletion patterns within the ISM and observations in the UV, optical, and IR. These models are either used as an ensemble of individual grain sizes, or integrated to give the opacity data of dust as a whole. Of course empirically based laws and templates are also often used in SED modelling such as the Milky way extinction law and Calzetti law (see below).

For the purposes of SED modelling and fitting, dust absorption and scattering, and dust emission are often treated as distinct components. As the hottest dust is constrained by sublimation to $\lesssim 2000 \mathrm{~K}$ (corresponding to $\sim 3-4 \mu \mathrm{m}$ peak emission), in practice only the scattering and absorption of light needs to be considered for modelling the optical-UV emission of galaxies. Conversely, as dust opacity strongly decreases with increasing wavelength, in the far-infrared (FIR) only dust emission needs to be considered.

Attenuation by dust The effects of dust on the optical-UV light are often described by two parameters - the reddening and total obscuration. Reddening is the wavelength dependence of dust effects, including features, and takes account of the fact that shorter wavelength photons are more readily scattered and absorbed by dust. This is often parametrized by the color excess $E(B-V)$ or the Balmer decrement $\mathrm{H} \alpha / \mathrm{H} \beta$. The total obscuration is a measure of the total light absorbed or scattered out of our -line-of-sight by dust either bolometrically or in a single band and can be considered the normalization of the reddening. This is generally parametrized as $A(V)$. For relative measures correcting only for reddening is sufficient, however for absolute quantities the total obscuration must also be taken into account. This is especially important when the reddening is close to flat, i.e. only small visible effects by dust on the spectrum.

For individual stars in the Milky Way, the Large and Small Magellanic clouds, extinction laws have been measured (e.g. Cardelli et al. 1989). However, when considering 
a galaxy as a whole, it must be taken into account that stars reside at different optical depths, depending on whether they lie on the side of the galaxy facing the observer or averted from the observer, and that the stellar light can be scattered into the observer's line-of-sight as well as out of it. Additionally, stellar populations of different age will have different extinction optical depths, and this extinction might have a different wavelength dependence. These issues lead to the concept of 'attenuation', where the complexity of the actual star-gas geometry is wrapped into a single attenuation law, now not applied individually to each star in the galaxy, but applied to the full spectrum of the galaxy.

Using an attenuation law, the dust obscuration of stellar light is expressed through a screen approximation (see Equation 2, as if the dust was lying between us and the stellar population of the galaxy, with a wavelength-dependent reddening law $\left(a_{\lambda}\right)$. The total amount of attenuation then depends only upon the thickness of the screen $(\Delta \tau)$,

$I(\lambda)_{\mathrm{obs}}=I_{\mathrm{star}}(\lambda) e^{-a_{\lambda} \Delta \tau}$.

The attenuation law was derived empirically for starburst galaxies by Calzetti et al. (1994); Calzetti (1997) who fit the law with a simple polynomial as a function of $1 / \lambda$. They found a law much greyer than the extinction laws of the Milky Way and LMC demonstrating the effects of geometry and mixing compared to simple extinction. Generally an simple power-law, $a_{\lambda} \propto \lambda^{-0.7}$, is able to reproduce the observed effective attenuation in galaxies (Charlot and Fall 2000).

However, a simple attenuation law cannot account for differential geometries and star formation histories within and between galaxies. This can be seen with the higher optical depths observed for nebular emission lines relative to the underlying stellar continuum, indicating that the stars and gas that give rise to the lines and to the continuum see different amounts of dust (Calzetti et al. 1994, Calzetti 1997). These observations led to the improvement over a simple attenuation law in the approaches of Silva et al. (1998) and Charlot and Fall (2000), who created a more physical twostep model in which young stars which emit ionizing photons are likely to be still surrounded by the clouds of gas and dust from which they formed. In this model all stars are attenuated by 'diffuse' dust in the same manner as equation 2. However young ( $<10 \mathrm{Myr})$ stars undergo an additional 'birth cloud' attenuation. In practice this means that the UV light and nebular emission lines associated with the short-lived massive stars are more obscured than the optical light dominated by the longer-lived stars, as observed in real galaxies.

While the empirically calibrated Charlot and Fall (2000) model is an improvement over a simple attenuation law, it still does not take account of the differential dust and star geometries that are clearly visible in resolved galaxies, such as bulges, disks, and dust lanes. The clumpiness of the ISM, both within the diffuse phase (see e.g. Kuchinski et al. 1998; Witt and Gordon 2000) and within the birth clouds (see e.g. Popescu et al. 2000; Dopita et al. 2005), will also affect the resulting attenuation of galaxies. However the greatest difficulty that simple, empirically-based attenuation laws face is the anisotropic scattering of light by dust, as photons are not only scattered out of the line-of-sight, but can also be scattered into it. This can cause bluer integrated spectra than can be accounted for by simple attenuation laws, especially for face on galaxies (see e.g Baes and Dejonghe 2001b; Fischera et al. 2003, Pierini et al. 2004, Inoue et al. 2006).

However, to take account of all these issues, proper radiative transfer (RT) calculations must be done, which require intensive computations. To limit these calculations several treatments exist, which can be broadly grouped into iterative methods and Monte Carlo methods (for a more detailed description for several of the methods used in RT calculations, see Baes and Dejonghe 2001a). In the iterative approach, the light is broken up into emitted and scattered components, with the RT equation solved separately for each component, and the solution from the previous component being used for the subsequent (i.e. directly emitted photons by stars, then photons scattered once by dust, photons scattered twice etc.) and these equations iterated to convergence (see e.g. Kylafis and Bahcall 1987; Xilouris et al. 1998, 1999, Tuffs et al.2004). Monte Carlo methods use a method closer to reality, where the paths of individual 'photons' are followed through their interactions (absorption and scattering) through the galaxy. The photons are emitted in a random direction from the sources, such as stars, and interact randomly with the surrounding ISM with a certain probability based on the mean free path length, and are followed through these scattering events until the photons escape or are absorbed. To build up an integrated SED of a galaxy, many photons must then be followed, though many treatments now exist to limit this number, such as only following photons which end up in the observer's line of sight (see e.g. Witt et al. 1992; Bianchi et al. 1996; Witt and Gordon 1996, for some early work on Monte Carlo RT in galaxies). Both of these approaches are currently used, with the iterative quicker for given geometries, while Monte Carlo is more able to handle complex distributions of stars and dust (several existing codes are discussed in the following section).

While obviously the most realistic approach, the limitation of the radiative transfer is that it requires complex calculations and thus it is not directly applicable to large sample of galaxies. RT codes have been used to provide template libraries of attenuation for a range of galaxies (Bruzual A. et al. 1988; Ferrara et al. 1999; Pierini et al. 2004), and also analytic functions for the attenuation of the components of galaxies (i.e. bulge, disk, clumps etc., Tuffs et al. 2004), 
to deal with this issue, yet these introduce several free parameters which may be difficult to determine for unresolved galaxies for which only broad-band SED is available. It is for these reasons that a simple attenuation law is still the most commonly used way to account for the effects of dust on the UV-optical SED.

One final note about the attenuation by dust is the silicate dust features that can appear in absorption at 9.7 and $18 \mu \mathrm{m}$. These features require large optical depths to be observed, and thus are generally only seen in galaxies with strong nuclear sources (i.e. nuclear starburst/AGN). As this absorption occurs againstmodeled dust emission, it is usually only modeled with a simple absorbing screen, otherwise it requires self-consistent radiative transfer (discussed in section 2.2.3.

Emission by dust Dust emission in the FIR and sub$\mathrm{mm}$ is most commonly modeled by a single black body $\left(F_{\mathrm{FIR}} \propto B_{\lambda}\left(\mathrm{T}_{\text {dust }}\right)\right)$ or emissivity-modified black body ( $\propto$ $B_{\lambda}\left(\mathrm{T}_{\text {dust }}\right) \lambda^{-\beta}$, also called grey body), or a simple sum over a limited (2-3) number of these. The first form assumes that all dust is in thermal equilibrium at one temperature $\mathrm{T}_{\text {dust }}$. The emissivity of dust grains is generally taken to be a power-law at these long wavelengths, with models and laboratory data suggesting indices ranging from $\beta=1.0-2.0$. Actually the $\beta$ index is expected to be a function of both grain size, composition and temperature (see e.g. Andriesse 1974, Draine and Lee 1984; Agladze et al. 1996, Mennella et al. 1998, with a nice discussion on the constraints on $\beta$ in the latter). When introducing more than one black body, one is generally limited by the number of wavelengths observed and the details of the model (see e.g. Dunne and Eales 2001). In general, two modified black-bodies are sufficient to model these wavelengths, encompassing the idea of warm and cold components of the ISM (see e.g. Popescu and Tuffs 2002 Hippelein et al. 2003, and the review by Sauvage et al. 2005).

In the MIR range simple black bodies are not sufficient and more detailed modelling is necessary. This is due to strong dust (PAH) emission features and the stochastic heating processes that become important for smaller dust grains. As the size of a dust grain decreases, the impingement of photons onto the dust grain surface becomes less frequent and more random, thus less statistically representative of the interstellar radiation field, allowing significant cooling between photon impacts (Figure 13 of Draine 2003). Thus, rather than having a single temperature, the dust has a range of temperatures and is parametrized rather by the strength of the radiation field heating it. To model this one can use either Monte Carlo calculations simulating the arrival of photons and subsequent emission, or more simply one assumes and solves for a steady-state distribution of temperatures given the strength and shape of the impinging radiation field and dust size and composition(see e.g. Guhathakurta and Draine 1989, Desert et al. 1990, Draine and Li 2007a). Once this temperature distribution is known, it can be convolved with black bodies modified by the dust emissivity in the MIR, including any features.

Polycyclic aromatic hydrocarbons (PAHs) could be either called the largest molecular species or the tiniest dust - emit strong features in the MIR (see e.g. Smith et al.2007). These features arise from specific bending and stretching modes of the large aromatic molecules (Bauschlicher et al. 2009). As PAH emission bands are so complex they are generally incorporated into the models by either assuming a template form for the MIR emission features (see e.g. Desert et al. 1990) or by modelling the physical processes in a way similar to the small dust grains (e.g. Weingartner and Draine 2001; Draine and Li 2007a). On the whole, while aromatic molecules within galaxies are accepted to be the source of the MIR features, the typical shapes, sizes, and ionizationcharges of these molecules are an active field of research.

More realistic FIR dust emission models must take into account that the dust within the ISM of galaxies will exhibit a range of temperatures, from the hot dust around young stars and in outflows to the coldest dust in cold molecular cores, driven by the range of radiation fields and dust sizes. Such complex emission models calculate, for a given radiation field, the emission from each grain size and composition and then integrate over these for a given dust distribution to obtain the total dust emission. The largest grains are generally considered to have a single temperature, as they will be in thermal equilibrium, leading to a simple distribution of temperatures dependent upon grain size and composition. In more accurate models, the smallest grains are considered to be stochastically heated and the temperature distribution of the individual grains is calculated (using, e.g., the treatment of Guhathakurta and Draine 1989). To finally calculate the IR emission from a galaxy, the distribution of dust masses over heating radiation field are also needed. Simpler IR emission models assume a functional form of dust mass over heating intensity; $d M_{d}=f(U) d U$, with $f(U)$ most often assumed to be a power law (see e.g. Dale et al. 2001; Dale and Helou 2002, Draine et al. 2007b). The most complex IR emission models use radiative transfer to calculate the radiation field distribution over a galaxy, where the distribution of dust and stars are assumed (i.e. parameters of the model), and thus these models directly link the dust absorption and dust emission. These are discussed in Section 2.2 .3 .

However, as the temperature distributions of the dust in the galactic ISM are dependent upon dust-gas geometry and cannot be determined from optical-UV data alone, empirically-based templates are often used for representing the IR SED of galaxies, especially when IR data is limited due to sensitivity or confusion. These templates take dust 
models as described above (i.e. multiple modified black bodies, or dust heated by a range of radiation fields) and match these to observed IR SEDs (or IR colors) of groups of galaxies. These templates then tend to have galaxy-wide properties such as IR luminosity or galaxy type as parameters, though intrinsic properties such as average interstellar radiation field intensity are also used. Well known examples of templates include those of Chary and Elbaz (2001), Dale and Helou (2002), Lagache et al. (2004), and, more recently, Rieke et al. (2009). Though these templates tend to be limited by the samples that define them, they provide a good alternative to models when no or very little information is available about the actual IR emission of a galaxy.

\subsubsection{Combining stellar and dust emission}

The full UV to IR SED of a theoretical galaxy can be created through the combination of the techniques and modelling discussed in the previous sections 2.1 2.2.2. However, the different wavelength regimes need to be consistently connected. The simplest method is to take the energy absorbed in the optical-UV (see Equation 2) and to distribute it across the MIR and FIR, assuming simple emission properties for the dust, such as black bodies. This is the method used by Devriendt et al. (1999) and da Cunha et al. (2008). These authors attempt to strike the balance between the capability to model large datasets and the minimum sophistication necessary for a realistic model.

To associate full UV-submm SEDs with their semianalytic models (discussed in the following section) Devriendt et al. (1999) created "STARDUST". This model assumes that stars and dust are homogeneously-mixed in the galaxy. The light from the stars, i.e. summed from SSPs, is then passed through an ISM with the amount of dust determined from a simple galaxy chemical evolution model. The dust-absorbed radiation is then re-emitted via a series of templates generated from the Desert et al. (1990) model and fitted to observed IRAS points, parametrized by the total IR luminosity.

da Cunha et al. (2008) follow a similar idea, but improve upon this by using the Charlot and Fall (2000) recipe for the attenuation. They thus obtain naturally corresponding 'birth cloud' and 'diffuse ISM' dust emission components over which the absorbed energy is distributed (see Figure 4). The two emission components are both made up of a PAH template and variable grey body contributions, with the birth cloud emission consisting of shorter wavelength (hotter dust) emission. Such a model can simultaneously determine quantities such as stellar mass and dust mass of a galaxy, and provide quantitative uncertainties for all parameters (see section 4.1). While this method is quick, and hence suitable for comparison against large datasets, it is self-consistent across the two emission components only in terms of the total amount of radiation absorbed and re-emitted; physical properties, such as the dust temperature or the shape of the emission within the components, are based on educated assumptions and are not constrained directly by the opticalUV absorption in the model.

A very similar method was followed by (Noll et al. 2009) with the CIGALE code, which uses either the Maraston (2005) or PEGASE codes for the stellar populations and only a Calzetti attenuation law to attenuate the stellar light. The major differences lies in the use of existing empirically calibrated templates, such as from (Dale and Helou 2002). rather than a free IR emission made up of several parametercontrolled components.

To be properly self-consistent, the absorption and emission must occur more 'simultaneously', such that the exact temperatures (including stochastic effects) of the dust causing the absorbing can be directly calculated. Such models require radiative transfer calculations to be performed, such that the exact radiation field, or at least the heating intensity, is known at each point in the dusty ISM. This, along with assumptions about the stellar ages and distribution, and the dust distribution and properties can then give the full UV-IR SED of a model galaxy.

The models of Efstathiou et al. (2000) and Siebenmorgen and Krügel (2007) do this radiative-transfer calculation using the ray-tracing method for starburst galaxies, which, being dominated by young stars and their birth clouds, are well represented by simple spherical approximations. These models build upon a strong history of dust radiative transfer and emission modelling and star-formation region modelling work to create simple models for the understanding of the UV-submm SEDs of starburst galaxies (Rowan-Robinson 1980, Rowan-Robinson and Crawford 1989; Rowan-Robinson 1992; Siebenmorgen and Kruegel 1992a; Siebenmorgen et al. 1992b, Rowan-Robinson and

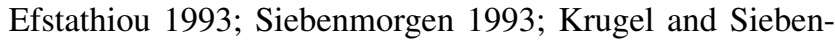
morgen 1994). These works are based on the observation that young stars are both relatively more luminous and more obscured (thanks to the birth clouds) than older stars, and that in strongly star-forming galaxies these young stars will be the dominant IR (and significant bolometric) sources. In particular, Siebenmorgen and Krügel (2007), reduce the results of complex modelling to a series of templates, based upon the physical properties of starbursting galaxies, such as the total luminosity, size and extinction of the star-forming regions and the contribution of the young stars to the total luminosity of the galaxy.

Groves et al. (2008) (building on previous works; Dopita et al. 2005, 2006a b), take this work a step further by self-consistently calculating the emission of a star-forming region, including the radiative transfer through the surrounding gas and dust simultaneously. Like Efstathiou et al. (2000) they allow for the $\mathrm{H}$ in regions to evolve over time, using empirically calibrated models. This model is well suited 
for modelling starburst (star-formation dominated) galaxies, where young stars and their 'birth clouds' dominate the emission, determining conditions such as star-formation rate and compactness of the gas and stars. Like da Cunha et al. (2008) and Siebenmorgen and Krügel (2007) it provides physical templates with as few parameters as possible. Yet, while it fits well the SEDs of star-formation dominated galaxies (see Figure 5), this model is not suited for nonstarbursting galaxies, where the distribution of the diffuse dust and stars must be accounted for.

By assuming a simple molecular cloud-disk-bulge geometry (as shown in figure 6), the GRASIL model (Silva et al. 1998, Granato et al. 2000) is able to account for the differential extinction suffered by the stars of different ages associated with each of these components in a galaxy. In addition, by varying the contribution of each component, galaxies from spirals to ellipticals can be modeled. Unfortunately, the more general geometry means that some parts (such as the gas-dust connection calculated in Groves et al. 2008) cannot be calculated, and also means more parameters are needed to define the model. As with the Groves et al. (2008) model, the more accurate dust calculations mean a longer calculation time, as compared with simpler models such as da Cunha et al. (2008). The GRASIL team is currently working on speeding up their calculations for semianalytic models (see following section) by the use of neural networks (Silva et al. 2010).

The main issue with all models discussed above is that, while they take account of absorption (and emission) reasonably well, they do not accurately take account of dust scattering, which, as discussed above, can make some galaxies appear bluer or redder depending upon inclination. This can be even more obvious in spatially resolved SEDs of galaxies, where light from stars which are obscured along our line of sight can be seen in reflection. However, as scattering is an inherently stochastic process, it is difficult to model simply in a galaxy, especially when multiple scatterings can occur.

Tuffs et al. (2004), following on from Popescu et al. (2000) and Misiriotis et al. (2001), use the iterative raytracing radiative transfer method of Kylafis and Bahcall (1987) to efficiently calculate the radiation field throughout model galaxies consisting of a stellar bulge, stellar and dusty disks and dusty clumps. Their resulting SEDs are then self-consistent across the UV-IR range. In addition, one of the strong benefits of radiative transfer is that the resulting SEDs can also be spatially resolved, and be compared to multi-wavelength studies of resolved galaxies, which they have done with edge-on galaxies such as NGC 891 (Popescu et al.2000) and NGC 5097 (Misiriotis et al.2001).

The other common approaches is to use the Monte-Carlo radiative transfer method to model the UV-IR SED of galaxies. Existing Monte-Carlo codes that have been applied to galaxies include SUNRISE (Jonsson 2006, Jonsson et al.

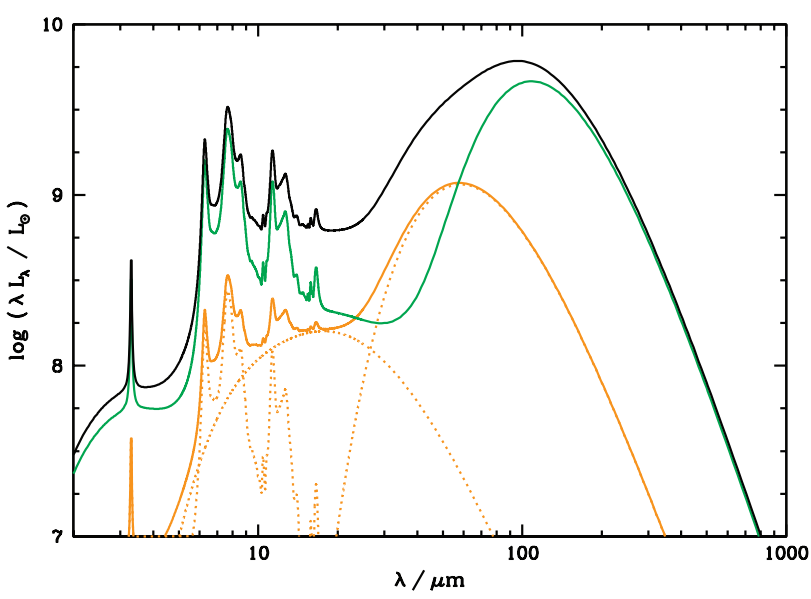

Fig. 4 IR emission of a simulated galaxy from the da Cunha et al. (2008) model (black curve) demonstrating the individual contributions from the 'birth cloud' dust (orange) and 'diffuse ISM' dust (green) [Courtesy E. da Cunha].

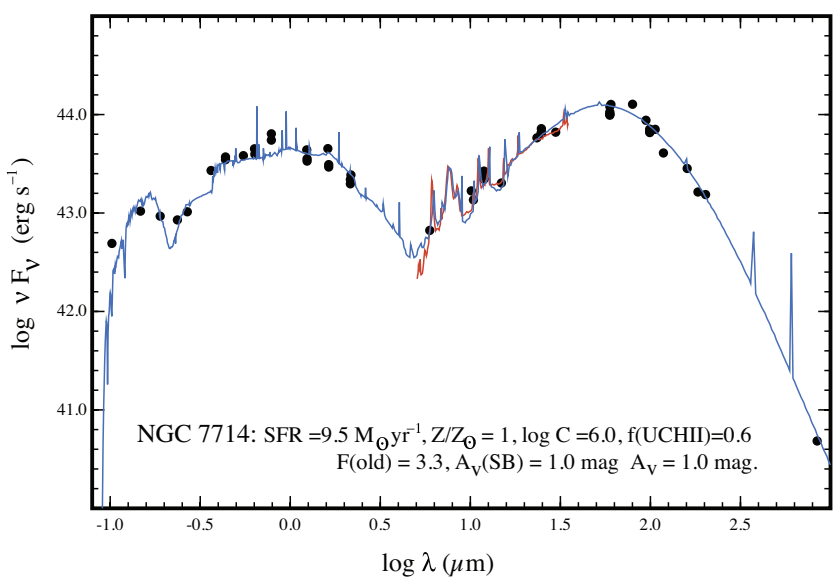

Fig. 5 Groves et al. (2008) model fit (blue curve) of the starburst galaxy NGC 7714 SED (black points and red curve mid-IR spectra), demonstrating the determination of physical galaxy properties such as star-formation rate (SFR) and metallicity (as labelled, see Groves et al.2008 for full description of parameters) [Courtesy M. Dopita]. 
2010), DIRTY(Gordon et al. 2001; Misselt et al. 2001), TRADING (Bianchi et al. 1996, 2000, Bianchi 2008), SKIRT (Baes et al. 2003), and RADISHE (Chakrabarti and Whitney 2009). These are able to model arbitrary and complex geometries of dust and gas, including spiral arms, dust lanes, bulges and clumpy ISM. However unlike the ray tracing method, the radiation field within the galaxy is not directly calculated (as only individual photons or photon packets are followed). Thus dust heating and emission must be treated through approximations (discussed in detail within the papers listed above). One treatment is to integrate within set volumes (i.e. a grid) the amount of energy absorbed by dust, and to redistribute this energy over large equilibrium grains. In some cases (Bianchi et al. 2000), small stochastic grains are also considered (using template assumptions). This approach can suffer from stochastic noise if the number of photons used is not sufficient. A similar treatment is to convert the absorbed energy into a radiation field using the dust cross-sections, and thus with the radiation field known the methods described in the Section 2.2.2 can be used (see e.g. Misselt et al. 2001, though this still suffers from issues of stochastic noise. Another treatment is called the "dust temperature update method". Here, the temperature of the grains is updated with the absorption and emission of each photon (described in detail in Bjorkman and Wood 2001 Baes et al. 2005). All these methods must iterate in the case of self-absorption of dust. A more efficient method iterates on the calculation of the radiation field density by using the previous estimates as a base and only calculating for the difference at each iteration. This method will always converge as each iteration only adds a small amount of dust emission which will provide an even smaller amount of dust emission. The new radiation field is converted to IR emission using models such as Dale and Helou (2002) or Draine and Li (2007a) (see e.g. Juvela|2005, Jonsson et al. 2010). While definitely more accurate in the treatment of dust, Monte

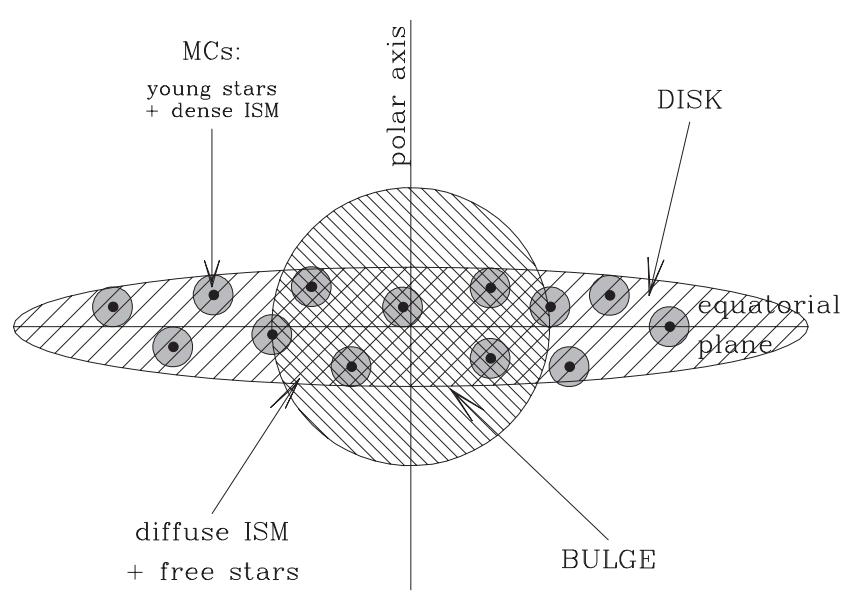

Fig. 6 Sketch of the geometry assumed within the GRASIL model (Figure 1 from Granato et al.2000).
Carlo codes require some representation of the ISM as input and are much more expensive computationally, especially in the cases where the dust is optically thick to its own (IR) emission and many iterations may be required. Current models are also, due to resolution effects both within the RT and galaxy models, unable to calculate the absorption on both diffuse (kpc) and local (pc) scales, and thus currently use approximations or sub-resolution models (see e.g. Jonsson et al. 2010). Hence, while reproducing "real" galaxies, they cannot be directly used to fit observations of individual galaxies.

In summary, the modelling of the transfer of stellar light through the ISM is well advanced, yet two significant challenges still exist. The first is simply the computational effort needed to represent the radiative transfer accurately. Many of the above models are limited in their resolution to trace the ISM accurately, and thus need sub-resolution approximations to treat some of the coldest or hottest dust (e.g. SUNRISE uses the starburst templates of Groves et al.2008) . The second is our general lack of understanding of the dust composition in the ISM. Generally, dust is assumed to consist mainly of carbonaceous and silicate-like grains (such as olivine), in some power-law size distribution, (see e.g. Mathis et al. 1977). This form is reasonably well constrained by observations of extinction in the optical-UV and emission features in the IR (see Draine 2003). Yet there are still open questions on shape (how ordered or "fluffy" are the grains, e.g. Zubko et al. 2004), on whether there are other kinds of dust, and on what formation and destruction processes lead to this power-law distribution of sizes (e.g. Jones et al. 1996). Conversely there are still spectral features associated with dust that are yet to be properly explained, such as the $2175 \AA$ absorption feature, the diffuse interstellar bands in the optical, and the "Extended Red Emission" band observed around 7000A (see Draine 2003, for a discussion on these features and other remaining issues).

\subsection{Evolution of Galaxies}

Together, the SSP and ISM radiative transfer models of the previous sections are able to reproduce the full UV-sub-mm SED of galaxies with a reasonably high degree of accuracy (see e.g. da Cunha et al. 2008; Groves et al. 2008). Yet, by themselves, these models are inherently static. Only limited model assumptions about the past evolution of the galaxy can be introduced through the star formation history. In particular, the ISM is rarely evolved along with the stars, and is presumed to be the same metallicity as the latest generation of stars in the SSP models. It is common form to assume that the dust in the ISM is a constant fraction of the metals within the gas, distributed in a form similar to that found in our Milky Way. 
These assumptions are sufficient to reproduce the observed SEDs of real galaxies using empirically based priors (e.g. Section 4.5), or multiple components (e.g. Section 4.4, and see Section 4 for full discussion). However to produce fully-theoretical SED models that are at least conceptually similar to local galaxies, one needs to fall back on galaxy evolution codes. There are three levels of these. At the innermost level are galactic chemical evolution codes, which, given some star-formation history and/or some "pristine" inter-galactic medium (IGM) infall rate, trace the evolution of the ISM metallicity, allowing for outflows, infalls, and pollution by stars (see reviews by Hensler 2008, Matteucci 2008). The more recent of these codes also evolve the dust along with the gas, taking into account the different pollution rates of different elements, and the evolving temperature/phases of the ISM (e.g. Calura et al. 2008). Once these codes have given the corresponding ISM evolution with the star-formation history (input or calculated), these can be associated with SSP and ISM codes to give a more self-consistent instantaneous spectrum of a galaxy (e.g. Schurer et al. 2009, Conroy et al. 2010a). Some of the main issues with these are the limited knowledge of the external gas losses and infalls, meaning that exact evolution cannot be obtained, and the computational time needed to calculate this evolution and associate it with a spectrum, meaning that only specific sets of SFH or infall can be calculated at a time.

The next scale above the chemical evolution models are models that evolve the whole galaxy. These models are based upon hydrodynamic and N-body codes that follow the evolution of the ISM and stars within a dark matter halo representing a galaxy (e.g. Springel 2005). These codes use empirically based relations to follow the detailed evolution, such as the formation of stars from gas, and the feedback from stars to the gas (see e.g. Tormen 1996, Cox et al. 2006). Containing both the stars (or "stellar particles") and the ISM (with known metallicity), these galaxy simulation/evolution codes are perfectly suited for linking with the Monte-Carlo radiative transfer codes such as SUNRISE (Jonsson 2006) or RADISHE (Chakrabarti and Whitney 2009) which have been purposely built to create spectra and broad-band images of these simulated galaxies.

The outermost layer are the cosmological models. These trace the formation of structure in the Universe from the original perturbations in the cosmic microwave background to redshift zero, using N-Body codes to simulate dark matter and its gravitational interaction (see Dolag et al.2008, for a review). While some of these models trace baryonic matter as well as the dark matter, most trace only the dark matter due to the more complex interactions of baryonic matter. Thus to trace the formation of galaxies within the forming dark matter halos semi-analytic models (SAMs) are used (e.g. Cole et al. 2000, Kauffmann and Haehnelt 2000, Hatton et al.2003, De Lucia et al.2004, Somerville et al.2008).
These models use the outputs from the dark matter simulations and approximate the physics of galaxy formation within the dark matter halos by empirical relations (e.g. for gas cooling, star formation, AGN fueling, feedback).

The SAMs return (and trace) the star formation history of each galaxy that is created, including the effects of mergers, as well as the gas content and metallicity of the gas (and stars). These results can be used in association with SSP models (as discussed in section 2.1) to determine the stellar spectra of each galaxy. As little geometrical information is returned by the SAMs, associating the ISM effects on the stellar spectra is more difficult, especially so for the IR emission. For the gas, most tend to use the associated emission lines added to the SSP models (see e.g. Leitherer et al.|1999. Charlot and Longhetti 2001). For dust attenuation, a simple treatment taken by many is to determine the extinction assuming a uniform mixing of the stars and gas in a galaxy, a fixed 'template' attenuation curve, and basing the optical depth on either empirical relations between galaxy luminosity (e.g. Kauffmann et al. 1999; De Lucia et al. 2004), or amount of dust in the galaxy (e.g. Guiderdoni and RoccaVolmerange 1987; Devriendt and Guiderdoni 2000). More advanced treatments include the use of the Charlot and Fall (2000) model (e.g. De Lucia and Blaizot 2007) or attenuation libraries like that of Ferrara et al.(1999), made for such purposes (e.g. Bell et al. 2003b).

For the dust emission, the situation is more challenging. The simplest treatments assume that all of the radiation attenuated in the optical (by the above treatments) are reemitted in the IR. This radiation is either distributed through modified Planck functions with empirically-calibrated temperatures (e.g. Kaviani et al. 2003) or empirically-based templates (e.g. Guiderdoni et al. 1998, Devriendt and Guiderdoni 2000). Yet such models do not take into account the strong geometrical dependence of dust heating or the strong variations in the spectral shape and they are clearly not self-consistent with the extinction in the optical-UV (see Section 2.2.3.

For self-consistent SED models, the SAMs need to be coupled with radiative transfer (RT) calculations such as GRASIL, which has been done only for a few models (e.g. Granato et al. 2000, Lacey et al. 2008). However one of the main strengths of SAMs is their computational efficiency and speed which allows the calculation of the physical parameters of the many galaxies in large cosmological volumes and over large redshift intervals for many different implementations of the galaxy formation physics. Yet RT is computationally intensive, and severely slows the SAMs, meaning only relatively small volumes were investigated in the SAM-RT models. In addition some of the details necessary for the RT calculations are generally poorly modeled within the SAMs. Thus currently there is a choice between poorly representative but fast, or better modelling and slow 
(see Fontanot et al. 2009, for an overview). The currently most advanced models choose to compromise by using a RTbased library, empirically linked with the SAMs (Fontanot et al. 2009) or even linked through artificial neural networks to account for the large and complex variations in galactic UV-IR SEDs (Silva et al., in prep).

Models of galaxy SEDs thus exist of varying resolution and complexity, adapted to model everything from individual galaxies or to large catalogs of galaxies on cosmological scales. While at each level of the SED models our knowledge of the important physical processes could be improved, SED modelling today is much more accurate across the wavelength range than it was even a decade ago.

\section{Constructing observed galaxy SEDs}

A major development in the last decade has been the advent of new observing facilities and large surveys at all wavelengths of the spectrum, enabling astronomers for the first time to observe the full SEDs of galaxies from the UV to the FIR, from the local universe out to redshifts beyond 6 . While databases such as those referenced in Table 1 make it tempting to simply go ahead and fit full galaxy SEDs, it is important to pause for a moment and review which are the difficulties associated with the construction of a single SED. Indeed, the "true SED" of a galaxy as defined in the models considers the sum of all photons emitted from inside the volume defining the galaxy. To make the observed SED of a galaxy, however, this "true SED" is then filtered through the spectral response curve of the instruments and is redistributed spatially over the point spread function (PSF). Additionally, the measurement process not only adds noise, but also makes it necessary to join data from different instruments.

The construction of multi-wavelength SEDS is a complex and rich subject and a dedicated review would be a welcome addition to the literature. In keeping with the scope of the present text we can here only give a very cursory treatment of the issue. As a starting point for further reading we suggest consulting some of the major multi-wavelength surveys and their overview articles provided in Table 1 below.

\subsection{Spectral response curve and resolution}

Be it in spectroscopy or in photometry, one identifies the SED as a series of wavelengths and associated fluxes. In both cases, this is only a simplification of the fact that the measurement process convolves the true SED with a spectral response curve, yielding a transmitted flux at an effective wavelength. In spectroscopy, the response curve is almost invariably assumed to be Gaussian, with a $\sigma$ determined by the slit width and the dispersing device. Therefore, in practice the distinction between the instrumental broadening and the broadening due to the intrinsic velocity dispersion of the astronomical object is not very sharp. To mimic the instrumental broadening, one should first convolve with the appropriate Gaussian and then resample onto the spectral bins. Care needs to be taken that the wavelength calibration of both models and data are better than a tenth of a pixel over the full wavelength range (this is not always the case, see e.g. Koleva et al. 2008).

In photometry, the response curve is much broader and therefore needs to be represented with more care, i.e. tabulated as a response function. The response function in turn depends on the detector quantum efficiency, the instrument transmission and the filter in use. Photometric calibration and response characterization is a vital task (see Koornneef et al. 1986; Landolt 1992, for just two prominent examples).

The signatures available for determination of the physical properties of galaxies of course depend on wavelength and on the achieved resolution. For example, in the optical many of the strongest features of galaxies can be adequately resolved at a resolution of $\mathrm{R}=\lambda / \Delta \lambda \sim 2000$, while the lowresolution part of the Spitzer IRS can easily resolve PAH features at $\mathrm{R} \sim 100$. However, spectroscopy is more expensive in terms of telescope time, making photometry very attractive for obtaining large samples. In the last decade, successful use of narrow-band filters have blurred the distinction between spectroscopy and photometry, see for example COMBO-17 (Wolf et al. 2003), COSMOS (Scoville et al. 2007) and NEWFIRM (van Dokkum et al. 2009). Narrowband filters have even been used to directly measure emission line equivalent widths (e.g. Kakazu et al. 2007).

\subsection{Spatial resolution, aperture bias and matching}

One of the main tasks when assembling a multi-wavelength SED, indeed any catalog that contains more than one measurement, is to control whether what is measured in each band is actually physically the same. Due to either the seeing of the atmosphere or the diffraction of the telescope, the flux from a point-like source is re-distributed over the point spread function (PSF) of a width that typically depends on the time of observation and on the wavelength used. Moreover, galaxies are intrinsically extended and their morphology may depend on the wavelength in which they are observed.

One of the main problems in the process of matching is the size of the PSF. Typically, the PSF is narrowest at optical wavelengths, while UV and IR PSFs are broader. This can lead to situations in which there is more than one optical counterpart to the UV, IR or sub-mm source. The agnostic way to deal with this is to simply exclude such objects from the sample, however, this may introduce a bias if the multiple optical counterparts are actually physically associated. A more intricate, but also more uncertain, way is to redistribute the flux according to optical priors (Guillaume et al. 
2006). Finally, the use of all available information, spatial as well as spectral, seems to provide a promising way forward for multiwavelength datasets (Roseboom et al. 2009).

Another problem related to the resolution of the telescope are aperture biases. A rather simple manifestation of this is that objects that are further away will be seen as smaller on the sky. Therefore, in order to construct comparable samples at different redshifts, one has to adapt the size of the extracted aperture to the same physical size. A more complicated problem is the definition of the "total light" from an object. Indeed, the surface brightness profiles (SBP) of galaxies usually extend much beyond the threshold observational surface brightness. In the case of specific objects, such as $\mathrm{cD}$ galaxies, these extended wings might contain a significant part of the total light from a galaxy (Oemler 1976, Carter 1977). Different strategies have been developed to avoid these biases, such as either integrating over a full model for the SBP or simply using specific apertures to integrate the light only inside some physical radius, but each method has its own problems. One of these is also that galaxies have different intrinsic morphology in different bands, thus complicating the application of consistent procedures, even when using data with comparable angular resolution.

A particular concern with fibre spectroscopic surveys such as the SDSS is that the fibre apertures (3" in SDSS) only sample part of the object, with this fraction different at each redshift. The effect of this can be tested by comparing result of a fit to the photometry of the whole galaxy against the photometric fit corresponding to the area of the fibre only (e.g Gómez et al. 2003; Brinchmann et al.|2004).

\subsection{Examples of multi-wavelength datasets}

We now describe three "real-life" examples of assembled multi-wavelength datasets. It would here be impossible to describe all existing datasets, we therefore chose just three that highlight different goals and methods.

\subsubsection{The Spitzer Local Volume Legacy - spatially resolved SEDs}

The Local Volume Legacy (LVL) is a Spitzer legacy program built upon a foundation of GALEX ultraviolet and ground-based $\mathrm{H} \alpha$ imaging of 258 galaxies within $11 \mathrm{Mpc}$. The goal of the LVL survey is to fill a vital niche in existing multi-wavelength surveys of present-day galaxies with a statistically robust, approximately volume-complete study of our nearest star-forming neighbors. Although star formation rates based on optical spectroscopy as well as GALEX ultraviolet and Spitzer infrared imaging have been measured for many thousands of galaxies, most currently available datasets are derived from flux-limited samples, and thus suffer from well-known biases against low-mass, low surface brightness systems. Multi-wavelength datasets that do include such systems often only provide representative samples of this galaxy population (e.g., SINGS; Kennicutt et al. 2003), and are thus not suitable for studies that both seek to probe the low metallicity dwarf galaxy regime and require datasets which are true to the statistics rendered by volumelimited sampling. LVL consolidates and builds upon recent Local Volume galaxy surveys which have acquired groundbased narrowband $\mathrm{H} \alpha$ (Kennicutt et al. 2008), GALEX ultraviolet (Lee et al., in prep.) and HST resolved stellar population imaging (Dalcanton et al. 2009), by collecting Spitzer IRAC and MIPS infrared imaging for a sample of 258 galaxies derived from these programs. The collection of these observations enable a wealth of spatially-resolved and spatially-integrated studies probing present-day star formation, chemical abundance, stellar structure, and dust properties as well as galaxy evolution, particularly for metal-poor, low-mass galaxies which dominate the LVL sample by number. The coupling of the infrared and ultraviolet data in this survey are explored in Section 6.2.3

\subsubsection{The Herschel ATLAS - unresolved SEDs}

ATLAS (Astrophysical Terahertz Large Area Survey) intends to provide the first unbiased survey of cool dust and obscured star formation in the local Universe. ATLAS will detect $\sim 250,000$ sources with a median redshift of $\sim 1$ over $\sim 550$ square degrees of sky in five bands covering the wavelength range $110-500 \mu \mathrm{m}$. The five photometric bands cover the peak of the dust SED for local galaxies and, crucially, can probe the cold dust component $(T \leq 20 \mathrm{~K})$ which makes up $\simeq 90 \%$ of the dust mass in most galaxies (Dunne and Eales 2001). Herschel can simultaneously provide an accurate measure of the bolometric far-infrared luminosity (related to the current star formation rate) and the total mass of dust (related to the gas mass). Figure 7 shows the improvements expected to be made to the measurements of the dust mass function and the need for a multi-wavelength approach to understanding the properties of galaxies.

The ATLAS will be unbiased as it does not rely on prior detection in other wavebands. Nevertheless, the ATLAS fields contain $>10^{5}$ redshifts and are the best studied fields of this size; they are the targets of surveys being carried out with GALEX, VST, VISTA, UKIRT and the South Pole Telescope and will be the natural targets of many future surveys, including ones carried out by DES, WISE, LOFAR and the two SKA precursor telescopes in the south.

The greatest challenge for the Herschel ATLAS is likely to be the matching of the submillimeter sources to the correct optical / infrared counterparts. At very low redshift this is fairly straightforward as the density on the sky of bright optical galaxies is low enough that associations are unlikely to be random. At intermediate-high redshifts the number of 

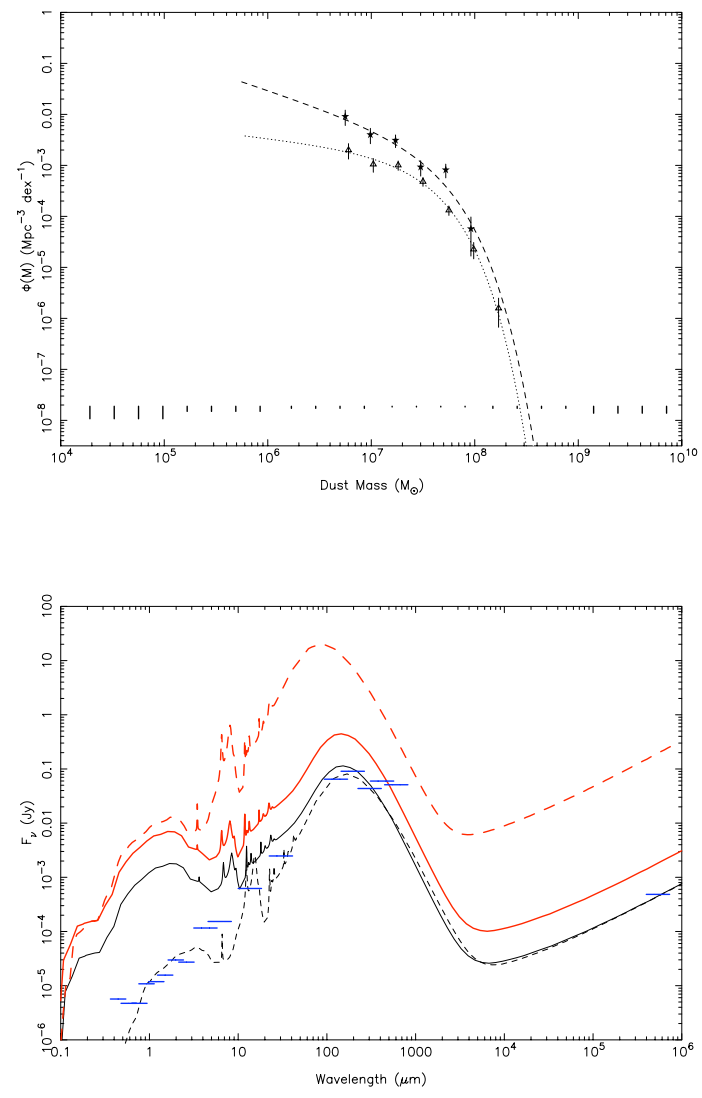

Fig. 7 Top: Recent estimates of the dust mass function from targeted observations of an IRAS sample (triangles; Dunne et al. 2000) and an optical sample (stars; Vlahakis et al. 2005). The error bars at the bottom show the accuracy and range for the dust mass function that will be measured with ATLAS. Bottom: Optical-radio SEDs from GRASIL (Silva et al. 1998) for M 51 (solid) - a typical spiral, and Arp 220 (dashed) - the archetypal ULIRG. Thick red lines show the SEDs at $z=0.05$ where they have a similar optical/ultraviolet flux but very different infraredradio SED. Optical / ultraviolet fluxes alone are therefore unreliable as a measure of a galaxy's star formation rate in dusty galaxies. Thin black lines show each SED at the redshift where it is just detected at $250 \mu \mathrm{m}$. This is $z \sim 0.1$ for M51 and $z \sim 1$ for Arp 220. The far-infrared-radio SEDs are now identical, while the optical fluxes are very different. Photometric redshifts from farinfrared/radio alone are totally unreliable without templates and optical / infrared fluxes to break the degeneracy between dust temperature and redshift. Horizontal dashes show the $5 \sigma$ flux limits of SDSS/UKIDSS-LAS/WISE/ATLAS and LOFAR all-sky surveys. [Courtesy L. Dunne] potential matches increases dramatically and the large Herschel beam (18-36") means that correct identification cannot simply be a matter of probability and distance. This will require a multi-wavelength SED modeling code which can 'join together' the ultraviolet / optical / near-infrared portion of the spectrum with the far-infrared/submillimeter 'bump' in a self-consistent way.

\subsubsection{The SWIRE templates}

Polletta et al. (2007) published a set templates that combine the SEDs of galaxies with those of AGN and thus fill an important hole in many SED fitting template set: ${ }^{2}$ The library contains 20 templates including 1 elliptical, 7 spirals, 3 starbursts, 6 AGNs, and 3 composite (starburst+AGN) templates covering the wavelength range between $1000 \AA$ and $1000 \mu \mathrm{m}$. The elliptical, spiral and starburst templates were generated with the GRASIL code (Silva et al. 1998). Templates of moderately luminous AGN, representing Seyfert 1.8 and Seyfert 2 galaxies, were obtained by combining models, broad-band photometric data, and ISO-PHT-S spectra of a random sample of 28 Seyfert galaxies. Four additional AGN templates represent optically-selected QSOs with different values of infrared/optical flux ratios and one type 2 QSO. The QSO1 templates are derived by combining the SDSS quasar composite spectrum and rest-frame infrared data of a sample of SDSS/SWIRE quasars divided in three groups, all, and the $25 \%$ brightest and $25 \%$ weakest measurements per rest-frame wavelength bin. The type 2 QSO template (QSO2) represents the SED of the red quasar FIRST J013435.7-093102 (Gregg et al. 2002). The composite $(\mathrm{AGN}+\mathrm{SB})$ templates are empirical templates that well reproduce the SEDs of the following sources: the heavily obscured BAL QSO Mrk 231 (Berta|2005), the Seyfert 2 galaxy IRAS 19254-7245 South (Berta et al.2003), and the Seyfert 2 galaxy IRAS 22491-1808 (Berta 2005).

\subsubsection{Further examples}

While we presented above only three examples, the number of public datasets (potentially) useful for SED fitting is truly staggering. Many science projects can be carried out without ever writing an observing proposal, the prime example being use of the SDSS database. This is possible thanks to the work of a countless number of individuals on survey planning, data reduction and quality insurance. Below we provide a table of some of the major datasets, for exploration and further reading. We will keep a similar table online on sedfitting.org and we hope to expand it in the future.

${ }^{2}$ The full library can be downloaded from: milano.inaf.it/ polletta/templates/swire_templates.html http://www.iasf- 
Table 1 Some major multi-wavelength databases

\begin{tabular}{|c|c|c|c|}
\hline Acronym & Full name & Reference & Website \\
\hline AEGIS & All-wavelength Extended Groth Strip International Survey & Davis et al. (2007) & aegis.ucolick.org \\
\hline COMBO-17 & Classifying Objects by Medium-Band Observations & Wolf et al. (2003) & www.mpia.de/COMBO \\
\hline COSMOS & Cosmic Evolution Survey & Scoville et al. (2007) & cosmos.astro.caltech.edu \\
\hline GAMA & Galaxy And Mass Assembly & Driver et al. (2009) & www.eso.org/ jliske/gama \\
\hline GOLDMINE & Galaxy On Line Database Milano Network & Gavazzi et al. (2003) & goldmine.mib.infn.it \\
\hline GOODS & Great Observatories Origins Deep Survey & Giavalisco et al. (2004) & www.stsci.edu/science/goods \\
\hline LVL & Local Volume Legacy & Dale et al. $(2009)$ & www.ast.cam.ac.uk/research/lvls/ \\
\hline MUSYC & Multiwavelength Survey by Yale-Chile & Taylor et al. (2009) & www.astro.yale.edu/MUSYC \\
\hline SDSS & Sloan Digital Sky Survey & Abazajian et al. (2009) & www.sdss.org \\
\hline SINGS & Spitzer Infrared Nearby Galaxies Survey & Kennicutt et al. $(\overline{2003})$ & sings.stsci.edu \\
\hline SSGSS & Spitzer SDSS GALEX Spectroscopic Survey & Treyer et al. $(2010)$ & www.astro.columbia.edu/ssgss \\
\hline SWIRE & Spitzer Wide-area InfraRed Extragalactic survey & Lonsdale et al. (2003) & swire.ipac.caltech.edu \\
\hline VVDS & Vimos VLT Deep Survey & Le Fèvre et al. (2005) & cencosw.oamp.fr \\
\hline
\end{tabular}

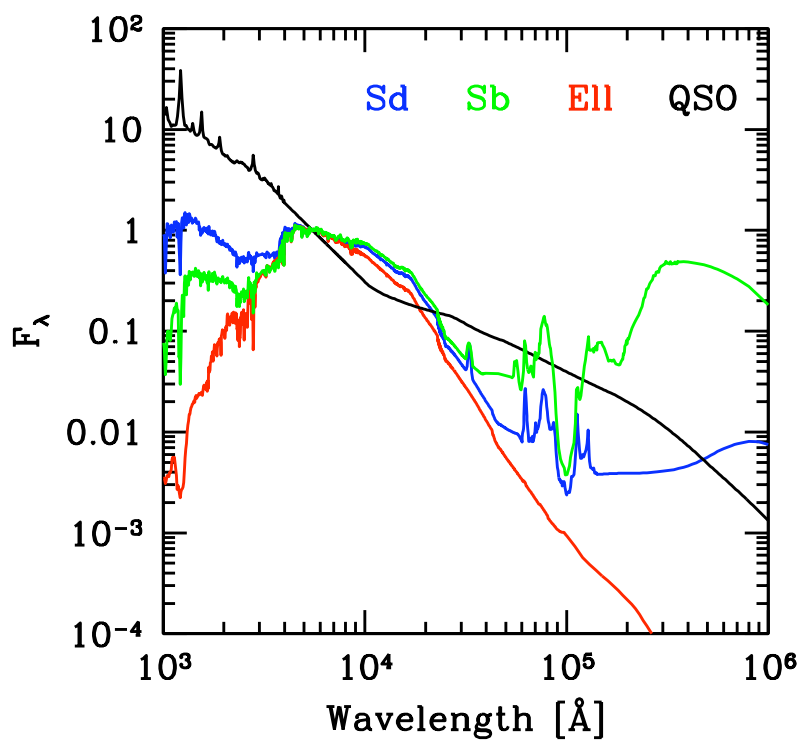

Fig. 8 Four of the 20 templates from Polletta et al. (2007), as an example of a set of empirically calibrated, multi-wavelength templates [Courtesy M. Polletta].

\section{Methods and validation of SED fitting}

In Section 2 we have described how models predict the SEDs of galaxies. Yet the ultimate goal of these models is to allow the reverse process, that is to derive the properties of galaxies from their observed SEDs through fitting procedures. Therefore we describe in this section several of the main methods used to fit model or observed template SEDs to multiwavelength observations of galaxies. Exactly which of these procedures should be used is contingent on which physical parameters are required and with what precision. In turn, this, and the accuracy with which the models reproduce specific observations, determines what observational data are necessary.

The following sections deal almost exclusively with stellar emission. This reflects a real dearth of specialized codes for carrying out the fitting process in the dust emission range (besides simple $\chi^{2}$ minimization over a parameter grid, e.g. Klaas et al. 2001). This state of affair is understandable, however, in light of the rapid development and large uncertainties still inherent to our modelling of the dust emission of galaxies.

\subsection{Parametrizing SED models}

In the context of a SED model, one must distinguish between independent, input parameters and derived parameters. Input parameters are those properties that are needed to define the SED model. Some of them may, first, not be measurable or, second, may not have a proper physical meaning by themselves. A good example for the first case is the shown in Figures 10 and 11 of da Cunha et al. (2008), where the width of the probability distribution functions of different input parameters indicates which ones are well constrained, such as the global contribution of PAHs to the total luminosity, and which are not constrained, such as the 
temperature of warm dust in stellar birth clouds. IglesiasPáramo et al. (2007), their Section 3) state another example, namely that only a few of the many input parameters of the GRASIL code need to be varied in order to produce a library of model spectra that cover a realistic range in observational properties. Further, more systematic study of the relative importance of the input parameters to dust emission models would seem to be a important addition to the literature. A good example of the second case is the "decay timescale", $\tau_{*}$ : in modelling the SEDs of early- and even late-type galaxies, the star formation histories (SFHs) are often represented as falling exponential functions modulated by this timescale, i.e. $\operatorname{SFR}(t) \propto e^{\left(-t / \tau_{*}\right)}$. However, in a hierarchical universe the SFH is likely to be more stochastic in form, modulated by discrete accretion events. Thus the width of the falling exponential that one determines from an SED fit is a good measure of the mean age of the galaxy. It should by no means be assumed that this represents the true SFH of the galaxy. The reason that falling exponentials provide reasonable fits in practice is simply that the spectral features of SSPs vary smoothly with time and there is thus considerable degeneracy between the mass contributions of SSPs of different age, effectively smoothing the SFHs of galaxies. This smoothing makes it also impossible in practice to robustly disentangle the epoch of the formation of the most luminous stellar population in a galaxy from the timescale over which this star formation took place.

Input parameters constitute a discrete set with a maximum number of members determined by the quality of the data and the models. On the other hand the set of derived parameters can be extended at will and not all derived parameters can or need to be independent. For example, the commonly used specific star formation rate $\left(\mathrm{sSFR}=S F R / M_{*}\right)$ and the birthrate parameter $\left(b=S F R /\langle S F R\rangle_{t}\right)$ are derived parameters that are very similar. It is clearly relevant to properly define and understand the derived physical properties that one intends to measure. Let us again consider the SFR. While the SFR derived from the UV flux traces an average SFR over the last $100 \mathrm{Myr}$, the SFR determined from Balmer emission lines measures the much shorter timescale of the ionizing stellar flux, $\lesssim 10 \mathrm{Myr}$. These two measures, while correlated, thus need to be properly corrected before being able to compare them (see e.g. Kennicutt 1998; Boquien et al. 2007; Kennicutt et al. 2009; Lee et al. 2009a. and references therein).

As an integrated model of the optical SED of a galaxy is based more or less explicitly on an equation similar to Equation 1, the independent and derived parameters should be defined in the same terms (see e.g. Walcher et al. 2008. Table 1). Actually writing down the definition helps avoid common confusions (such as those between metallicity $\mathrm{Z}$ and $[\mathrm{Fe} / \mathrm{H}]$, or concerning the time scale over which the SFR is measured) and should be considered good practice.

\subsection{Spectral indices}

It has been said in the introduction that SED fitting can only yield useful results if the models are as or more precise than the effect on the data of the property to be measured. Historically this was the case only for very limited wavelength ranges in the optical. The solution to this problem has then been to not fit the entire SED, but to define indices, i.e. measure the equivalent widths, for certain absorption features.

In the standard side-band method (Burstein et al. 1984; Faber et al. (1985) a careful analysis leads to the definition of one central and two side bands (a blue and a red). The continuum compared to which the equivalent width is measured is a linear interpolation between the average fluxes found in these two sidebands. Much work has gone into optimizing the set of available indices, as well in terms of coverage, as well as in terms of model precision (e.g. Rose 1984; Worthey et al. 1994; Trager et al. 1998, Tripicco and Bell 1995; Cenarro et al. 2002; Thomas et al. 2004; Lee et al. 2009b, and references therein).

We are still waiting for standard indices in other wavelengths than the optical, though see Rix et al. (2004), Keel (2006), Maraston et al. (2009), \& Chavez et al. (2009), and Lançon et al. (2008) \& Cesetti et al. (2009) for headway into the UV and NIR respectively. These would make the index approach a true multi-wavelength approach.

Rogers et al. (2010a) have attempted to improve on the classical sideband definition by introducing a "boosted median" method. The main feature of this method is that for each side-band only the $L$ th percentile of the distribution of fluxes is used to determine the flux within the side-band. As this procedure automatically chooses the largest fluxes in the side-band, it will define the pseudo-continuum from those points that are least affected by "secondary" absorption features, i.e. absorption features that are not part of the central band. It thus improves the robustness of the pseudo-continuum if the spectral resolution is high enough to avoid blending of all features. Figure 9 illustrates the difference between the boosted median equivalent width and a standard side-band method for a measurement of $\mathrm{H} \gamma$. The sample corresponds to a set of 14 elliptical galaxies in the Virgo cluster observed with a 2-2.4A resolution (FWHM) and with high $\mathrm{S} / \mathrm{N}$ (Yamada et al.|2006).

Index fitting can be considered a special case of SED fitting (for a fitting code see e.g. Graves and Schiavon 2008). It has the advantage of compressing the information available in galaxy spectra into a set of discrete numbers. Much of what is considered secure knowledge in stellar element abundances and age of integrated stellar populations is still largely based on fitting indices (e.g. Trager et al. 2000 , Kauffmann et al. 2003a; Thomas et al. 2005; Gallazzi et al. 2008: Graves et al. 2009, to cite only a few).

The clear disadvantage of absorption line indices is that some of the information is lost. While indices have been 
defined with great care to approach the ideal of representing one element species, in practice many small lines interfere in particular in the side-bands. Spectral fitting (Section 4.4) can in principle use more information but it places much higher requirements on model accuracy.

\subsection{Principal Component Analysis}

Ideally we would like to represent a galaxy spectrum by a small set of continuous parameters that uniquely determine the best-fit spectrum. Principal Component Analysis (PCA) is one algorithm commonly used to derive an optimal set of linear components, diagonalising the covariance matrix of the data points to find the directions of greatest variation. Its representation of data through a linear combination of independent (orthogonal) components, or eigenvectors is thus an alternative method to using a set of discrete SSP templates (Section 4.4). Since the convolution with transmission curves is a linear operation, these methods are as simple as solving a linear equation, even for photometric datasets (Connolly et al. 1999, Budavári et al. 2000).

PCA has been successfully applied to astronomical spectral datasets, although not yet to photometric datasets which suffer the additional complication of observed-to-rest frame translation (Connolly et al. 1995b). The main difficulty with PCA is that the interpretation of the empirically determined PC components in terms of physical properties is complex at best (though see Wild et al. 2007, 2009, Rogers et al. 2007, $2010 \mathrm{~b}$ ). This is exacerbated by its sensitivity to outliers and hence out-of-the-box algorithms are of limited use for astronomical datasets.

Recent work by Budavári et al. (2009b) solves the problem of reliable eigenspectra determination by an iterative procedure that is efficient to compute and robust in the statistical sense. Figure 10 illustrates the comparison of three PCA methods on the blue optical region of 2000 randomly selected SDSS galaxy spectra. Each column contains (from left to right) the results of the classic PCA, classic PCA using iterative sigma clipping of the dataset, and the new robust algorithm. What is immediately striking in the robust case is the following: (a) The very clean appearance of the nebular emission lines in the second eigenspectrum. (b) These are correlated with the weaker Balmer absorption (seen as broad wings on the narrow emission lines) and rise in the blue from the continua of $\mathrm{O}$ and $\mathrm{B}$ stars which are the dominant source of ionisation of the nebular emission lines. (c) The emission line in the 4th eigenspectrum is the only one in this wavelength range which is not a Balmer line, and with a higher ionisation state is sometimes attributed to the presence of an AGN. Without any prior physical knowledge, the robust PCA has separated out a line which is physically distinct from the others, and tied together HII emission lines with the $\mathrm{O}$ and $\mathrm{B}$ stars that excite them. The results are

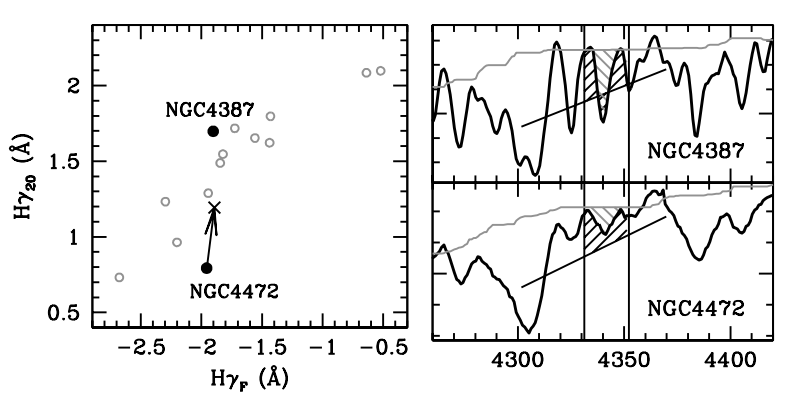

Fig. 9 Comparison of the $\mathrm{H} \gamma_{F}$ equivalent width measured with the side-band (SB) and the proposed Boosted Median method $\left(\mathrm{H} \gamma_{20}\right.$; BMC). The black dots mark two galaxies with a significant difference between both methods. On the right-hand panels, the SED of these two galaxies is shown, with the slanted line representing the continuum defined by the SB method, and the gray line showing the BMC pseudo-continuum. The hatched black (gray) regions correspond to the equivalent width according to the side-band (BMC) method. The arrow and cross in the left panel shows the change in equivalent width caused by the difference in velocity dispersion between NGC $4387(112 \mathrm{~km} / \mathrm{s})$ and NGC $4472(303 \mathrm{~km} / \mathrm{s})$. [Courtesy I. Ferreras]

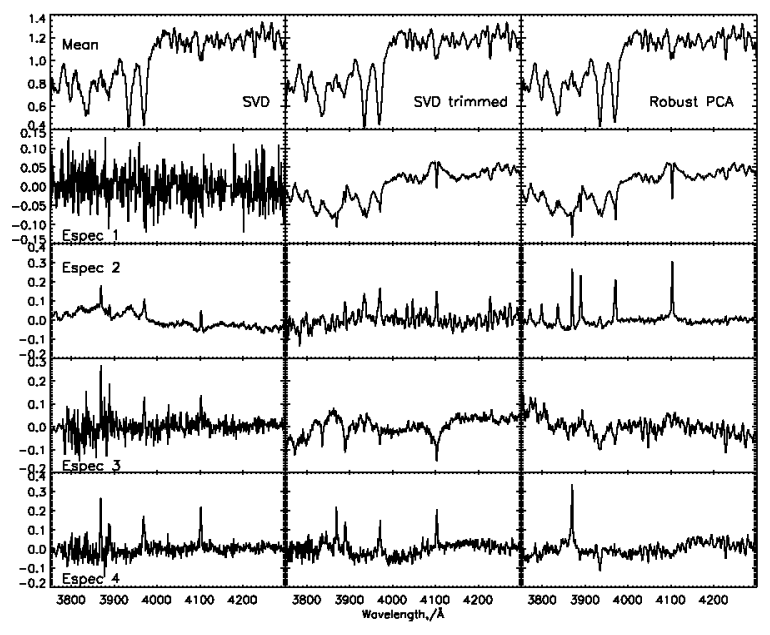

Fig. 10 Comparison between the eigenspectra from a PCA of 2000 randomly selected SDSS galaxies: left, using a simple SVD algorithm, the first eigenspectrum is dominated by a single noisy galaxy spectrum; center, using sigma clipping to remove outliers iteratively from the dataset, the eigenspectra are visibly less noisy; right, a robust PCA of the dataset, now the distinct patterns in the dataset are strongly visible, in particular the separation of narrow nebular emission lines from broader photospheric absorption features. [Courtesy V. Wild] 
clearly of more use for characterising the galaxy population than traditional PCA algorithms. The robust PCA algorithm provides a new, fast and easy to use method for the investigation of real astronomical datasets in a model independent manner.

\subsection{Spectral fitting by inversion}

As has been mentioned in Section 2.1.1 the stellar SED of a galaxy can be represented by a sum over the SEDs of individual SSPs with appropriate weights which reflect the SFH. As long as any complications, such as dust, can be neglected this is a linear problem, i.e. a matrix inversion. More generally, inversion is the attempt to invert the observed galaxy SED onto a basis of independent components (SSPs, dust components) drawn from a SED model. Inversion is typically used for spectral data and a big success of modern stellar population models and inversion codes is that we can now fit the models to data to better than $5 \%$ in the optical wavelength range (see Section 4.4.4).

\subsubsection{Method}

Nearly all inversion methods start with assumptions that reduce the complex physics of SEDs (see Eq11) to a problem that can be written as a linear function of its parameters. Such assumptions are typically that one deals with a stellar system in which all generations of stars have the same metallicity, i.e. $\zeta(t)=Z^{0}$ and the same attenuation, i.e. $T(t)=T^{0}$. The problem of solving for the star formation history of a stellar system is then equivalent to defining and minimizing the merit function

$\chi^{2}=\sum_{i=0}^{n}\left[\frac{F_{i}-\sum_{i=1}^{M} a_{k} S_{i}\left[t_{k}, Z^{0}, T^{0}\right]}{\sigma_{i}}\right]^{2}$,

over all non-negative $a_{k}$. Here $F_{i}$ is the observed spectrum in each of $n$ wavelength bins $i, \sigma_{i}$ is the standard deviation and $a_{k}$ are weights attributed to each of $M$ SSP models $S_{i}\left[t_{k}, Z\right]$ of age $t_{k}$ and metallicity $Z$. This merit function is linear in the $a_{k}$ and can thus be solved by standard mathematical methods involving singular value decomposition (e.g. non-negative least squares, bounded least squares ${ }^{3}$ A particular advantage of the inversion method is that, besides the assumptions necessary to linearize the problem, no parametrization of the solution is necessary, in particular not of the star formation history. Codes that have been used for scientific analysis are now common and include MOPED (Heavens et al. 2000), PLATEFIT (Tremonti et al. 2004), VESPA (Tojeiro et al. 2007), STECKMAP (Ocvirk et al.

\footnotetext{
${ }^{3}$ It needs to be emphasized here that a correct solution involves a simultaneous fit for the velocity dispersion of a galaxy. This is beyond the scope of the present review though.
}

2006), STARLIGHT (Cid Fernandes et al. 2005), sedfit (Walcher et al.2006), NBURSTS (Chilingarian et al.2007), ULySS (Koleva et al.2009). Different codes give very comparable results (e.g. Koleva et al. 2008).

There is one problem that has to be addressed when assessing the unparameterized information content of galaxy spectra. All methods relying on singular value decomposition and derived algorithms inherit one of the features, which is that the method tends to search for the smallest number of templates it can use to fit the data. For galaxies, which presumably have a smooth SFH, this feature is a grave caveat concerning the significance of the recovered weights of each population. In particular, the recovery of realistic error bars from a simple bootstrap algorithm is not possible, as the method will tend to always choose some templates over others, inside a range of random errors. One way to address this issue is regularization, detailed in Ocvirk et al. (2006 the STECKMAP program).

Another robust exploration of this issue is provided in the code VESPA (described in detail in Tojeiro et al. 2007). As independent parameters (see 4.1) Tojeiro et al. chose 2 values of metallicity and a logarithmic binning in age that can be varied between coarse ( 3 age bins) and fine (16 age bins). Dust attenuation and varying metallicity are explored by repeating the fit with VESPA over a grid of parameters. Error bars are derived by creating noised representations of the input spectra and repeating the fit $n$ times. The codes main feature in the present context is that it explores the SFHs in an iterative process that goes from coarse to fine resolution. It uses the method described in Ocvirk et al. (2006) to estimate at each step, how many parameters can be recovered for a linear problem perturbed by noise. The best fit will thus only use as many independent stellar populations as required by the data. Nevertheless, one caveat remains, which is that the SFH inside each bin is fixed to be either a constant SFR or such that the light contribution of each age is more or less constant. When recovering parameters such as $\mathrm{M} / \mathrm{L}$ and in those cases where the age bins are coarse, this will lead to an underestimate of the true uncertainty in this parameter, as compared to a truly non-parametric method.

The main lesson learned from this careful analysis is that from spectra typical of the SDSS $(\mathrm{S} / \mathrm{N} \approx 20$, optical wavelength range), one can recover between 2 and 4 stellar populations described by an age and a metallicity. These values can be improved by a factor of 2 when going either from $\mathrm{S} / \mathrm{N}=20$ to $\mathrm{S} / \mathrm{N}=50$ or when enlarging the wavelength range to include not only the optical, but also the UV range. Such data will be available in the near future from the XSHOOTER instrument at the VLT. It should be kept in mind, however, that while the formal constraints may be better, the accuracy of the results will then depend critically on the data calibration and model accuracy over a very large wavelength range. 


\subsubsection{Non-linear inversion codes}

For the optical wavelength range, an exception to the pre-condition of linearity exists in the form of the code STARLIGHT (Cid Fernandes et al. 2005), which is based on simulated annealing and thus does not need linearity. Consistency between STARLIGHT and other codes based on linearity assumptions has been found. Another code avoiding linearity conditions was presented in Richards et al. (2009).

For decomposing MIR spectra into their stellar, PAH, dust continuum, and line emission constituents, LevenbergMarquardt algorithms have been used (Smith et al. 2007. Marshall et al. 2007, see Section 6.2.2. This allows among other things to compute uncertainties on the derived parameters. With the improvement of dust emission models and in particular the more widespread availability of spectral data, more development in the technicalities of fitting dust spectra can be expected.

Finally, a possible extension of the inversion onto SSP models has been presented in Nolan et al. (2006) and would merit further exploration. Also Dye (2008) has presented a new method, which integrates inversion into a Bayesian framework and applies it to photometric data.

\subsubsection{Non-linear physics}

Dust attenuation is important in the optical and UV wavelength ranges. In the context of inversion, the importance of dust is most easily assessed by an iteration over a grid of attenuation values. More sophisticated schemes proceed in an iterative way, i.e. alternate linear inversion and a nonlinear minimization scheme (Koleva et al.2009), or use nonlinear minimization schemes for all parameters (Cid Fernandes et al. 2005). Any estimate of the dust attenuation at optical wavelengths from the SED alone (i.e. using neither Balmer decrement, nor UV photometry, nor dust emission) is bound to be uncertain, in particular because no inversion code at present uses a physical model for different attenuations likely experienced by young and old stars.

A very useful way is therefore to normalize the continuum of the observed and model spectra before the fit (e.g. Wolf et al. 2007; Walcher et al. 2009) or even simultaneously with the fit (Koleva et al. 2009). While this takes out any information related to the attenuation, it also allows the freedom from any uncertainties related to continuum calibration and can be thought of as fitting the equivalent widths of the absorption features only. An often under-appreciated caveat is, however, that this procedure throws away some information. In particular (nearly) featureless continua (such as from very young stars) become undetectable, yet still affect the equivalent widths. This "featureless" continuum has to be appreciated in relation to the noise in the observations and, in an intermediate age spectrum can even include a significant contribution of old stars. One expression of this caveat is described in Section 4.6 as the bias occurring when fitting single SSP models to extended SFHs.

Also the effects of forward scattering on the optical SED are not included in Equation 1 and cannot be linearized usefully. In principle the scattering can be modelled through radiation transfer modelling and could thus be included in non-linear minimization schemes. However, radiation transfer modelling is too time-consuming to make this a practical possibility.

\subsubsection{Validation}

In an unpublished contribution to the workshop, Jarle Brinchmann (and collaborators) showed that the $10^{6} \mathrm{spec}-$ tra of galaxies in the Sloan Digital Sky Survey (SDSS) offer a rich set of tests of the current models and techniques for fitting SEDs in the optical region. For the first series of tests they use a set of 274613 galaxies from the SDSS DR7 with reliable determinations of the emission line fluxes. They fit them using the latest version of the Bruzual and Charlot (2003) models (termed here CB08). In the optical the main improvement in these models is the switch from the Stelib library to the MILES library. The fitting procedure involves classical inversion using a non-negative least squares routine. Additionally they allow for $\sim 150 \AA$ wide mismatches between models and spectrum using a smooth final correction. This is necessary because of uncertainties in the spectrophotometry of the data and the models. The result of the NNLS routine is, among others, the mean $\chi^{2}$ of the spectrum, which can in turn be plotted into the classic Baldwin et al. (1981, BPT) diagram [NII] $\lambda 6584 \AA / \mathrm{H} \alpha$ ver-

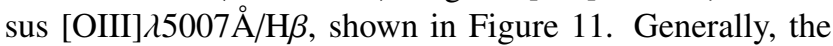
fit is good, with a reduced $\chi^{2}$ around $1 \pm 0.1$. Regions can be identified, where the fit is, while still in this range, somewhat worse. These are (A) galaxies with high star formation activity, high excitation and low metallicity, (B) strong, high excitation AGN, and (C) galaxies with high surface mass density (i.e. high mass) and weak emission lines.

For cases (A) and (B), i.e., the low metallicity starbursts and the high-ionization AGN, they relate the main increase in $\chi^{2}$ to the imperfect masking of some emission lines and to the lack of nebular or AGN continua in the spectra. In addition the BC03 models have some problems with hot stars (e.g. Wild et al. 2007) - the preliminary version CB08 appears to work better, due to a better coverage of hot stars in the spectral library (G. Bruzual, priv. comm.).

For case (C) one of the largest mismatches in the optical is located, as expected, around the $\mathrm{Mg}$ features at $5100 \AA$. This is the region where the enhancement of the abundances of the $\alpha$ elements is known to play an important role. $\alpha$ enhancement is not covered by the CB08 models. Nevertheless, in the optical the mismatch due to stellar populations is modest, of the order of 0.02 magnitudes. 
The best method to validate a fitting procedure is to compare the result to independent measurements of the same physical property. This can be done for redshifts, as discussed in section 5. but also for the spectra of nuclear clusters, as done by Walcher et al. (2006) for nine nuclear clusters. The nuclear clusters have multi-aged stellar populations and are as such similar to entire galaxies, and their total stellar masses can be determined dynamically, independent from fitting the stellar spectra.. As shown in Walcher et al. (2006), the stellar M/L ratios from inversion agree with those determined from independent dynamical modelling, albeit with a large scatter. This scatter reflects the general difficulty of constraining the oldest stellar population from SED fitting. Additionally, Walcher et al. (2006) also found that the age of the youngest population as determined from the spectral fitting is correlated to the measured emission line equivalent width, as shown in figure 12 (right). On the other hand, the mean mass-weighted age is not correlated, which is evidence that the fit result is not heavily biased towards the youngest population (in contrast to results from fitting single SSPs, see section 2.1.2.

Another careful work on validation of the spectral fitting procedures was the work by Koleva et al. (2008), who looked at Galactic clusters which had individual stellar spectra and full colour-magnitude diagrams to compare with the results of the fits to the integrated spectra (also compare this with section 2.1.2 for the validation of the predictions of SSP models for integrated spectra).

\subsection{Bayesian inference}

We refer here in particular to using the "Bayesian fitting" method, in which multi-wavelength SEDs are fit by first precomputing a discrete set or library of model SEDs with varying degrees of complexity and afterwards determining the model SED and/or model parameters that best fit the data. This approach is favoured in particular for multi-wavelength data, as the problem of solving for the physical parameters is not linear if effects such as dust attenuation, line emission, and dust emission have to be taken into account. In addition, the influence of geometry and the physical conditions of the dust generally make the number of parameters very large, and with many possible degeneracies, thus making non-linear minimization schemes usually impractical.

\subsubsection{Method}

It is important to realise that the simple fact of precomputing a set of galaxy models and afterwards determining the one with the lowest $\chi^{2}$ is by itself a Bayesian approach. By choosing which models to compute one introduces a prior, which, while possibly flat in terms of a given parameter, assumes that the data can be represented by that

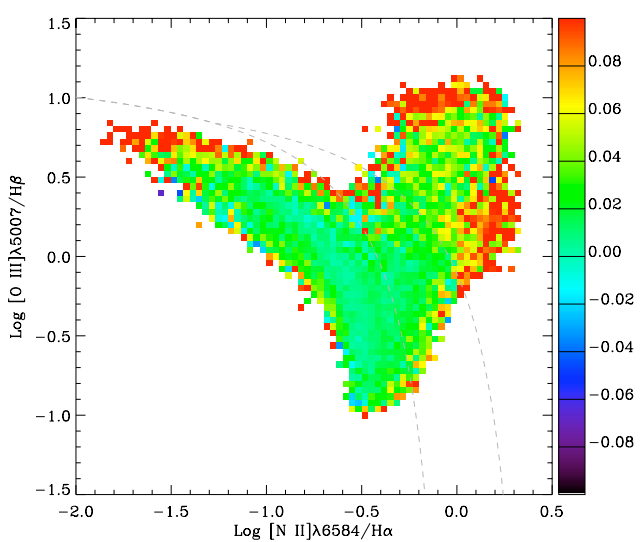

Fig. 11 Classic BPT diagram of $[\mathrm{NII}] / \mathrm{H} \alpha$ versus[OIII $] / \mathrm{H} \beta$, showing the distribution of SDSS emission line galaxies. The colours indicate the median $\log \chi^{2}$ of the fit of the CB08 model library to the SDSS fibre spectra with those emission line ratios. The upper and lower dashed lines indicate the Kewley et al. (2001) and Kauffmann et al. (2003b) AGN dividing lines respectively. [Courtesy J. Brinchmann]

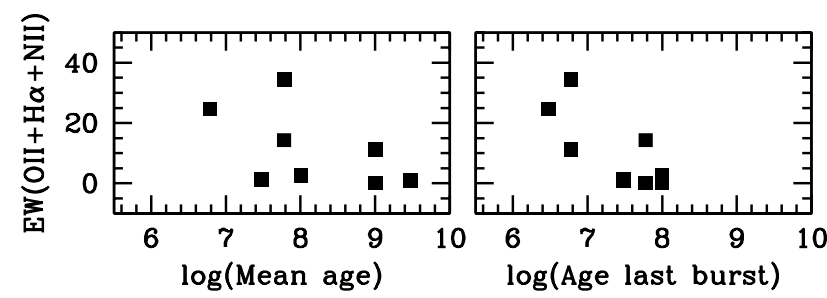

Fig. 12 Comparison between the equivalent widths of emission lines and the mean age (left panel) or the age of the last burst (right panel) as determined from spectral fitting for a sample of nine nuclear clusters from Walcher et al. (2006). The clear correlation is evidence that the youngest age picked up by the spectral fit does bear significance. 
model and that parameter space. By using $\chi^{2}$ as a maximum likelihood estimator one finds the most probable model, or in bayesian terms, the probability of the data given that model. A mathematically much more rigorous description of the method is given in Appendix A of Kauffmann et al. (2003a) and currently builds the basis for Bayesian inference in SED fitting. A more practically oriented description can be found in e.g. section 4 of Salim et al. (2007). In a nutshell, the method uses the fact that - assuming Gaussian uncertainties - the probability of the data (D) given the model $(M)$ is given by $\mathrm{P}(\mathrm{D} \mid \mathrm{M}) \propto e^{-\chi^{2} / 2}$. The prior probability distribution of the model parameters is often taken to be flat within a given parameter range (or flat in log space for larger parameter ranges). To determine the parameters for a specific galaxy dataset, the probabilities for all models are computed and then integrated over all parameters (model space) except the one to be derived, which yields a Probability Distribution Function (PDF). The median and width of this distribution yield the parameter estimate and associated uncertainties. The integration can be repeated and the PDF can be built for all parameters of the models as well as the observables themselves, e.g. with the aim to plot the model and observed fluxes in a fitted band for comparison purposes.

An important step in Bayesian inference for SED fitting is the construction of the library and the prior assumptions (Section 4.5.2). It is equally important to synthesize the correct observables from the models, taking into account response functions and redshifts (Section 3.1).

Bayesian inference for SED fitting has several advantages that minimize potential sources of uncertainties: i) all available measurements contribute to the fit result, ii) the $K$-correction is integrated, iii) non-linear effects such as from dust are accounted for as part of the models (see section 2.2.2, and thus in a self-consistent way, and finally iv) uncertainties on the derived parameters include measurement uncertainties as well as intrinsic degeneracies. The big caveats concerning this method are the sensitivity to the prior distribution of parameters (the "library") and, connected to this, the dependence on realistic input physics in the modelling.

\subsubsection{Libraries and priors}

The computation of the library is one of the essential steps when using Bayesian inference. It encodes our prior knowledge about the galaxies in the sample, but also our assumptions about which of the physical effects we can safely neglect. Concerning photometric SEDs, a prominent case of neglection is the internal chemical evolution of the model galaxies. This is justified because even the overall metallicity is not measurable from broad-band photometry alone (e.g. Walcher et al.|2008). Also the effects of forward scattering on the optical SED are often not included for computational reasons, but could potentially be important, in particular for starbursts (Jonsson 2006).

One assumption that is generally used is that SFRs tend to decrease monotonically with cosmic time in a given object (with the exception of star formation bursts). This assumption is particularly appropriate for early-type galaxies, which has led to the wide-spread use of so-called $\tau$-models, i.e. exponentially falling functions representing the SFH. However, it is not to be expected that galaxies in reality follow the falling exponential model. The SFHs of dwarf galaxies are expected to be dominated by intermittent bursts (see e.g. Gerola et al. 1980). But even for large galaxies, the SFH is not expected to be as smooth as suggested by the simple $\tau$-models. Both semi-analytic (Quillen and BlandHawthorn 2008) and hydrodynamic modelling suggest some stochasticity, as discussed in section 4.1. However, due to the smooth variation of SED properties with age and due to the ensuing degeneracies, the $\tau$-models provide reasonable fits to observed SEDs and cannot be easily refuted. Also, they provide a convenient, simple parametrization of SFHs which explains their widespread use.

The danger of using only $\tau$-models lies in the neglect of degeneracies. Let us assume, the $\chi^{2}$ of a specific $\tau$-model is the same as that of a different model, which is the superposition of a smooth SFH and a burst. Both models will have different mean ages and $\mathrm{M} / \mathrm{L}$ ratios. If they are both included in the library, they will thus lead to a larger error bar. However, if the burst model is omitted, two situations are possible: 1) If we indeed know that strong secondary bursts are unlikely (as e.g. for a sample of morphologically selected early-type galaxies) one will obtain a better estimate of the actual error bar through use of a better prior. 2) If on the other hand one is fitting the entire population of galaxies in a given volume, it is unlikely that no galaxy shows a secondary burst and one will thus - sometimes severely - underestimate the true uncertainty of the recovered parameters. Often "new", supposedly more precise estimates of galaxy parameters are published that are based on too restrictive priors. In the following we showcase two examples of how to construct a library of star formation histories.

A library for Gaia One of the first cases where Bayesian inference is applied to spectra will be presented in Tsalmantza, et al., (in prep). This is also a good example of how to create a library with a suitable prior for a specific application. The Gaia mission will produce spectra of $10^{6}$ galaxies at low resolution of R between 50 and 200. Automatic classification of a large number of low-resolution spectra is therefore crucial. Classification is done by comparison to a custom-created library of model galaxy spectra that simulate the properties of the Gaia spectra. This 
library is used for training and testing of the classification and parametrization algorithms.

Two libraries have been created: The first library is an entirely synthetic one. The library creation starts from the synthetic spectra of 8 typical galaxies produced by PEGASE.2 (Fioc and Rocca-Volmerange 1999). The SFHs of the library spectra are built from analytic prescriptions for either the dependence of SFR on the gas mass (SFR $=M_{\text {gas }}^{p 1} / p 2$, late types) or for the SFH directly (SFR $=p 2 . e^{-t / p 1} / p 1$, early types) as well as parameters for gas infall and galactic winds. In order to create a smooth grid, the input parameters (i.e. $p 1, p 2$, gas infall and galactic winds) were allowed to vary over a range of values(see Tsalmantza et al. 2007 . 2009). At this point, while of course guided by prior knowledge of the properties of galaxies, the parameter distribution is chosen nearly ad-hoc.

A second library was created based on spectra from the SDSS (DR5, Adelman-McCarthy and et al. 2007). The SDSS spectra were shifted to $\mathrm{z}=0$ and degraded to the resolution of the PEGASE. 2 output spectra. They were then classified by comparison to the pre-existing library of purely synthetic spectra through simple $\chi^{2}$-minimization. Using the best fit model spectrum the SDSS library spectra could be extended to cover the Gaia wavelength range and values for the most significant parameters could be assigned to them. Mainly, however, the results of the $\chi^{2}$ fitting were used to judge how the synthetic library compares to the empirical galaxy population in the SDSS. The distribution of input parameters governing the SFHs obtained from the observed SDSS sample can then be used to determine a suitable prior distribution for the synthetic Gaia library. This application is a good example of a case where the prior can be well established based on other observations.

Simplifying $M / L$ determinations A good example of how the appropriate choice of prior can help with constraining the fit is provided by the work of Bell and de Jong 2001, and $R$. de Jong's talk at workshop). This work centers on finding the simplest way to derive the mass-to-light ratio of a stellar population. Let us first remember that the stellar mass itself is the product of a galaxies luminosity and its $M / L . M / L$ in turn is a function of the star formation history of a galaxy. If the SFR at time $t$ is written as $\Psi(t)=d M(t) / d t$ and is defined over the entire Hubble time $T_{h}, L_{v}(t)$ is the luminosity of an SSP at frequency $v$, at age $t$, and with mass $d M(t)$ and $T_{v}(t)$ is the mean transmission of the ISM at wavelength $v$ and for the SSP with age $t$, then we can write

$M / L_{v}=\frac{\int_{0}^{T_{h}} d t \Psi(t)}{\int_{0}^{T_{h}} d t L_{v}(t) T_{v}(t)}$

This dependence of the $M / L$ on the SFH means that any prior assumption on the SFH is also a prior assumption on the $M / L$.
The crucial simplification inherent in Bell and de Jong (2001) is thus the parametrization of the SFH. This simplification is provided by a prior derived from a hierarchichal formation scenario. Using their scenario, the SFHs of galaxies lead to a situation in which their intrinsic colours show a strong correlation with $\mathrm{M} / \mathrm{L}$. The dust attenuation vector is parallel to this relation, and thus the $\mathrm{M} / \mathrm{L}$ ratio can be derived from a single optical colour with reasonable precision. Nevertheless there is a curvature in the difference between the $\mathrm{M} / \mathrm{L}$ ratios determined from colours only and $\mathrm{M} / \mathrm{L}$ ratios determined from an SED fit in the sense that for very blue (young) galaxies and for very old (red) galaxies, the colour-M/L will provide an overestimate, while for intermediate colour, intermediate age galaxies the colour-M/L will be an underestimate.

De Jong \& Bell (talk at workshop) explore different possible effects. When comparing a smooth $\mathrm{SFH}$ with a single burst, the $\mathrm{M} / \mathrm{L}$ values have large systematic offsets, due to the lack of faint, old stellar populations in the single burst model. Two burst models do not solve the problem, in particular if the fit is dominated by two young populations. In this case the two-burst model is still not complex enough, it again misses the faint, old population, which is crucial for the mass budget. The problem with the SFH template is further clarified by a comparison between $\mathrm{M} / \mathrm{L}$ ratios as determined in Bell et al. (2003a) and those derived by k-correct (Blanton and Roweis 2007). While the scatter in the difference is relatively small and there is no systematic trend, there is a systematic offset of about $0.15 \mathrm{dex}$. This can traced to the parametrization of the SFH. Indeed, while Bell et al. (2003a) use pure falling exponentials, the SFH assumed in in k-correct peaks $6 \mathrm{Gyr}$ after the beginning of star formation. Thus, while the resulting SED is not affected, the number of low-mass stars is much higher in the Bell et al. (2003a) SFHs. This uncertainty of 0.15 dex is thus inherent to any mass estimate from SED fitting.

\subsubsection{Validation}

In order to validate the method and its intrinsic accuracy, a number of studies have been performed in the last years. What could be termed "internal validation" is the search for degeneracies and systematics in the models and the method itself (see e.g. Walcher et al. 2008; Wuyts et al. 2009, Longhetti and Saracco 2009).

Extensive testing of stellar mass determinations through SED fitting was done with the COSMOS dataset (Ilbert et al. 2010). SEDs were generated with the stellar population synthesis package of Bruzual and Charlot (2003) assuming a Chabrier (2003) initial mass function (IMF) and an exponentially declining star formation history with a timescale, $\tau_{*}$, ranging from 0.1 to $30 \mathrm{Gyr}$. The SEDs were generated on a grid of 51 timesteps between 0.1 and 13.5 Gyr. Dust attenuation was simulated using the Calzetti et al. (2000) law with 
$E(B-V)$ in the range 0 to 0.5 , with steps of 0.1 . The colors predicted by this library of SEDs in the COSMOS dataset for the photometric redshift of the galaxy were compared to the observed ones to select the best-fitting templates.

How the potential inaccuracy of the photometric redshifts affects the stellar mass derivations was investigated, and results are displayed in Figure 13. Two spectroscopic samples were used: the zCOSMOS bright spectroscopic sample (Lilly et al.2007) selected with a magnitude cut only at $i_{A B}^{+}<22.5$ and a spectroscopic follow-up of $24 \mu \mathrm{m}$ sources selected from S-COSMOS (Sanders et al. 2007). These sources are typically dusty starbursts with $18<i_{A B}^{+}<25$. A median difference smaller than 0.002 dex is found between the photo- $z$ and the spectro- $z$ stellar masses, for both spectroscopic samples.

It is interesting to note that the stellar synthesis models have the same effect for both selections, as shown in Figure 13 This is not the case when studying the impact of the attenuation law. The MIPS selection consisting preferentially of dusty galaxies, the difference due to the choice of attenuation (the Calzetti et al. 2000 law in a case, the Charlot and Fall 2000 in the other) is amplified for this sample. The median difference in stellar masses is $-0.1 \mathrm{dex}$ for the optical selection and -0.24 dex for the infrared selection.

The impact of the choice of stellar synthesis model on the stellar masses determinations was also examined. Figure 13 presents the difference between stellar masses computed using the Bruzual and Charlot (2003) models and the new models of Charlot and Bruzual (2007, private communication) including a better treatment of the pulsing asymptotic giant branch. A median difference of 0.13-0.16 dex and a dispersion of 0.09 dex is observed. Pozzetti et al. (2007) found a similar difference (0.14 dex) when comparing Bruzual and Charlot (2003) and Maraston (2005) models. Walcher et al. (2008) show that this offset changes with the measured sSFR of the galaxy, in the sense that the masses determined using $\mathrm{BC} 03$ for objects with an intermediate sSFR $(-12<\log (\mathrm{sSFR})<-9)$ are, in the mean, more massive by $50 \%$ than those determined using CB07. These are the objects where the TP-AGB stars are most likely to contribute significantly to the light in the NIR bands. Objects with lower or higher sSFRs do not show this offset in Walcher et al. (2008).

Ilbert et al. (2010) concluded from this study that the use of photometric redshifts is only a weak source of uncertainty when deriving stellar masses for a sample of galaxies. The uncertainties are dominated by the uncertainties in the SED modeling itself, thus one has to be very cautious about the interpretations when selecting samples where a specific type of model is preferred.

External validation of the method is possible for one parameter in particular, namely the SFR. Using the entire SED to derive the SFR was considered less precise than other
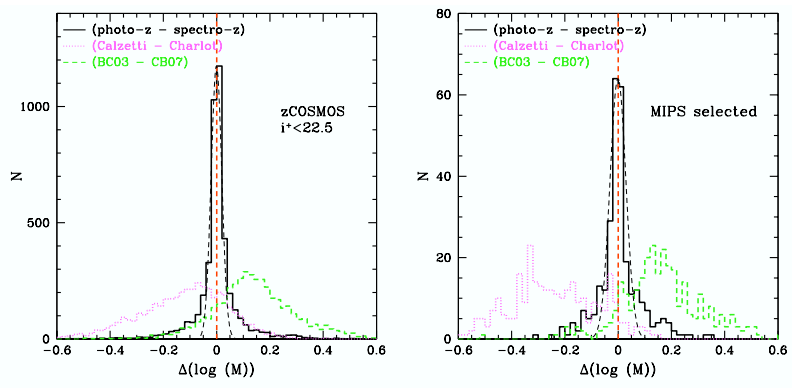

Fig. 13 Histograms of the difference between two stellar mass determinations, for the zCOSMOS bright sample selected at $i_{A B}^{+}<$ 22.5 (right) and for a sample of sources selected at $24 \mu \mathrm{m}$ in the S-COSMOS MIPS sample (right). The black histograms show the difference in stellar masses when using the photometric or the spectroscopic redshifts. The thin black dashed lines are gaussian distributions with $\sigma=0.02$ (left panel) and $\sigma=0.03$ (right panel). The green dashed lines show the differences between the stellar masses computed with Bruzual and Charlot (2003) and Charlot \& Bruzual (2007, private communication). The magenta dotted lines show the differences between the stellar masses computed using the Calzetti et al. (2000) and Charlot and Fall (2000) attenuation laws. The redshifts were set to the spectro- $z$ values in the two last cases. Systematic uncertainties due to the models dominate the errors introduced by the photo- $z$, at least in the magnitude/redshift range explored with our spectroscopic samples. [Courtesy H. Aussel] 
methods (based on specific tracers) in Kennicutt (1998). The availability of large samples with UV, optical and NIR photometry have lead to a reappraisal of the method in the last years. Salim et al. (2007) show a detailed comparison based on the data from the SDSS and the Galex satellite. They derive SFRs using the multi-wavelength SED. They then compare these to the SFRs derived by Brinchmann et al. (2004), which used all emission lines that can be usefully measured in the SDSS spectra and detailed modelling of the emission line spectrum to derive dust-corrected, emission-line based SFRs. As shown in their figure 6, the SED-derived SFRs show in general a satisfying agreement. A similar test was performed in Walcher et al. (2008) on a VVDS-SWIREGALEX-CFHTLS sample, where the SED SFR was compared to SFRs derived from the [OII] $\lambda 3727 \AA$ emission line. While the latter are much less accurate than the emission line SFRs from Brinchmann et al. (2004), the agreement between SED and emission line SFR was shown to be satisfying out to a redshift around one (their figure 8). An advantage of obtaining SFRs from broad-band SEDs compared to emission-lines is the time needed to obtain sufficient $\mathrm{S} / \mathrm{N}$ in the spectra to get the lines. Nevertheless, an interesting residual correlation of SFR difference with stellar mass is found in SED fitting, which remains yet to be fully understood. Another interesting problem exists in early-type galaxies, in which no $\mathrm{H} \alpha$ emission can be measured, yet UV emission is commonly found (see e.g. Salim et al. 2007, Figure 3). This probably is the cause why some objects with no measurable emission lines have relatively high SED SFRs.

In order to test the SFR determinations from SED fitting at $\mathrm{z} \sim 1$, Salim et al. (2009) extended previous work to include the mid-IR dust emission. The consensus is that the mid-IR emission is heated by the UV, and hence traces the emission of young stars ( $\lesssim 100 \mathrm{Myr}$ ) and it therefore provides a good means to measure the SFR. Deep MIPS $24 \mu \mathrm{m}$ imaging exists for the Extended Groth Strip (EGS), as well as redshifts assembled in the context of the DEEP2 survey. The rest-frame $12 \mu \mathrm{m}$ emission was converted to a total dust luminosity using the Dale and Helou (2002) prescriptions. Using the dust attenuation determined from fitting the UVoptical SED one can calculate the dust-corrected UV and B-band luminosities. These correlate well with the $24 \mu \mathrm{m}$ luminosity as seen in figure 14 Turning the IR luminosity into a SFR using the relations of Kennicutt (1998), the best correlation was found for an SED-SFR measured over the last 1-3 Gyr. That the mid-IR may be heated by older stars recently received support in a study by Kelson and Holden (2010), which predicts that C-type TP-AGB stars ( 1.5 Gyr old) can produce a large fraction of the mid-IR flux.

Nevertheless, for some very red objects in their sample, some SED SFRs seriously underpredict the SFR determined from the IR emission. These galaxies have a dominant old population, where the IR may have little to do with SF. Indeed, for the case of early-type galaxies Johnson et al. (in

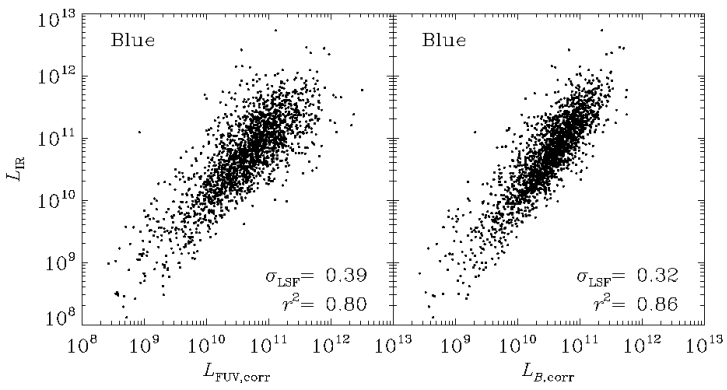

Fig. 14 Comparing UV and optical luminosities to the IR luminosity determined from the $24 \mu \mathrm{m}$ flux. The offset $(\Delta)$ and dispersion $(\sigma)$ around the mean relation of the galaxy sample are marked in the lower right corner. Figure from Salim et al. (2009) [Courtesy S. Salim]. 
prep.) find that it is important to consider the fraction of the stellar emission absorbed redwards of $4000 \AA$ in order to obtain a good prediction for the measured FIR emission. Neglecting this emission leads to an underprediction of the FIR emission by factors of up to 5 .

Stellar mass is the second parameter that can in principle be calibrated empirically, with the hope of deriving the normalization of the $\mathrm{M} / \mathrm{L}$ ratio, in other words the choice of IMF. However, uncertainties related to technical questions concerning the SED fitting and the dynamical mass measurements are still too high to derive very accurate overall IMF normalizations (see e.g. Cappellari et al. 2006, Salucci et al. 2008). Careful attention should also be paid to issues such as the contribution of dark matter, recycled gas, and other dark components. In the formulation of Bell and de Jong (2001), the slope of the colour-M/L relation is independent of the IMF, but the normalization depends on it. For this reason Bell and de Jong (2001) used the maximum disk approximation to provide an upper boundary of the total mass in stars allowed in spiral galaxies. They thus confirm that the Salpeter IMF leads to too high $\mathrm{M} / \mathrm{L}$ ratios and they adapt the normalization of the colour-M/L relation to be $0.15 \mathrm{dex}$ below Salpeter. Thus a Kroupa or Chabrier IMF seem to be good choices (see section 2.1.1). Variations between stellar population models and in dependence on the available data range also need to be considered carefully (see e.g. van der Wel et al. 2006). As many more independent mass tracers exist, which require careful assessment, the interested reader is pointed to de Jong \& Bell (in prep.) for further discussion of this topic.

\subsection{Method-independent caveats}

A good example of how the quality of the available models and data influence the use of tracers and the precision with which physical properties can be recovered is given by recent developments in the use of index fitting. Historically, early-type galaxies have been analyzed using simple stellar populations models as templates, effectively using the prior assumption that early-type galaxies were created in one single burst of star formation. However, detailed spectroscopic studies (Trager et al. 2000) as well as near-ultraviolet photometry of early-type galaxies (Ferreras and Silk|2000; Yi et al. 2005; Kaviraj et al. 2007) have confirmed the presence of hot stars in early-type galaxies.

Figure 15 illustrates that it is now possible and necessary to go beyond SSPs for early-types (see also e.g. Serra and Trager 2007). The marginalized age distribution is shown for four galaxies corresponding to three different models (from left to right): Simple Stellar Populations (SSP), a 2burst model consisting of a superposition of an old and a young SSP (2BST), and a composite model assuming an exponentially decaying star formation rate, including a simple prescription for chemical enrichment (CXP). All these models combine the population synthesis models of Bruzual and Charlot (2003). The figure shows that SSPs give mutually inconsistent age distributions, whereas composite models such as 2BST or CXP give a more consistent picture. Notice how lower mass galaxies such as NGC 4489 or NGC 4239 (top panels) give better fits for a 2 burst scenario, whereas higher mass galaxies (NGC 4464, NGC 4387; bottom panels) are better fit by a smooth star formation history.

A similar analysis is presented in Trager and Somerville (2009), who analyze mock data from semi-analytic models in standard ways, in particular computing the "SSPequivalent" ages and metallicities. They quantify that the SSP-equivalent age is poorly correlated with the massweighted or light-weighted average ages. The SSP-ages tend to be younger, biased to the star formation in the last 0.1-2 Gyr. This has in particular the effect of exaggerating the signature of downsizing. On the other hand, the SSP-equivalent metallicity is mostly equivalent to the lightweighted metallicity. This serves as an important reminder, that the prior assumption that one puts into the analysis are very important for the resulting measurement. In the case of using SSP-equivalent ages, the assumption, or prior, is that galaxies are single-age entities. This inevitably leads to important biases in the determined parameters.

Among the important caveats of SED fitting, the issue of the age-metallicity degeneracy cannot remain unmentioned. This degeneracy is particularly important in the optical. In a nutshell, age effects on colors or line strengths can be mimicked by a change in metallicity. This is especially important in the old populations found in early-type galaxies. Equally important and generally well-understood are the effects of using the optical SED only to determine the attenuation. Basically this is a very difficult undertaking and under almost any circumstance, measurements of the Balmer decrement, the FIR emission or the UV slope are essential to a reliable determination of the attenuation.

In principle it should be possible and informative to combine information from the photometric SED and spectral information (Gallazzi and Bell 2009, Lamareille 2009). While widespread use of this combination is hampered by possible systematic differences in the results (e.g. Wolf et al.|2007; Schombert and Rakos 2009), general agreement between both types of information has been claimed at least for stellar masses (Drory et al. 2004). Brinchmann et al. (Talk at workshop) showed the results of a systematic comparison between photometric and spectral SED fitting. Comparing formal errors, they find no significant systematic offset. However, for actively star forming galaxies it might be better to use multiwavelength, broad-band photometry than the detailed spectrum information. The reason is, as mentioned in Section 4.4.3 that very young stars tend to "hide" in the normalized optical part of the spectrum, however they are 
readily visible as blue continuum in the multi-wavelength SED. On the other hand, spectra are better at picking up recent bursts through their Balmer lines (e.g. Wild et al. 2007, 2009). Emission lines are a potential problem for fitting photometric SEDs. A quick back of the envelope calculation, however, shows that they produce a maximal offset in r-i colour of 0.1 at an equivalent width of $100 \AA$. Thus, ELs are a minor issue in the broad-band colours when a full SED is available and of limited concern for normal $\mathrm{z} \sim 0$ galaxies ${ }^{4}$.

Galaxies with significant redshifts can in principle be treated the same way as local ones, subject to two main caveats though: (1) we currently do not have the same kind of information available for large samples of galaxies at redshifts above 1 as for local galaxies. (2) Galaxies at high redshifts may have been significantly different from todays galaxies, e.g. concerning their typical star formation histories, their metallicities or their gas content. As local analogs are rare or lacking, SED models are less well calibrated and may be subject to considerable systematic uncertainties. These need to be explored in detail, which is currently only possible through semi-analytic models (see e.g. Schurer et al. 2009). The design limits of SED fitting codes should thus be kept in mind when quoting results and - in particular - errorbars on high redshift properties.

\section{Results of SED Fitting: Photometric redshifts}

A special case of analyzing SEDs of extragalactic sources is the problem of redshift estimation, a topic that is usually refered to as photometric redshifts (hereafter photo-z). This problem is distinct from all other estimates of physical properties because independent and more precise measurements of the same property are available for large samples in the form of spectroscopic redshifts. The method can thus be tested extensively and even calibrated empirically. It is also one of the earliest forms of SED fitting, having been suggested as a manner to go beyond the limits of early spectroscopy (Baum 1957).

For a working definition, Koo (1999) suggests the following: "photometric redshifts are those derived from only images or photometry with spectral resolution $\lambda / \Delta \lambda \lesssim 20$. This choice of 20 is intended to exclude redshifts derived from slit and slitless spectra, narrow band images, rampedfilter imager, Fabry-Perot images, Fourier transform spectrometers, etc." This definition leaves room for a wide variety of approaches that are actively being explored by members of the community. While today most studies build on a set of magnitudes or colors, recently other observables have been utilized with good success, e.g., in the work by Wray

${ }^{4}$ But recall that equivalent widths are proportional to $(1+\mathrm{z})$ and that the sSFR also tends to increase with redshift. and Gunn (2008). However, all methods depend on strong features in the SEDs of the objects, such as the Balmer break or even PAH features (Negrello et al.2009).

Traditionally, photometric redshift estimation is broadly split into two areas: empirical methods and the templatefitting approach. Empirical methods use a subsample of the photometric survey with spectroscopically-measured redshifts as a 'training set' for the redshift estimators. This subsample describes the redshift distribution in magnitude and colour space empirically and is used then to calibrate this relation. Template methods use libraries of either observed spectra of galaxies exterior to the survey or model SEDs (as described in Section 2). As these are full spectra, the templates can be shifted to any redshift and then convolved with the transmission curves of the filters used in the photometric survey to create the template set for the redshift estimators.

Both methods then use these training sets as bases for the redshift estimating routines, which include $\chi^{2}$-fitting and various machine learning algorithms (e.g. artificial neural networks, ANNs). The most popular combinations are $\chi^{2}$ fitting with templates and machine learning with empirical models. For a review of the ideas and history of both areas, see Koo (1999).

The preference of one over the other is driven by the limitations of our understanding of the sources and the available observations. Template models are preferred when exploring new regimes since their extrapolation is trivial, if potentially incorrect. Empirical models are preferred when large training sets are available and great statistical precision is required. Here we review these techniques and estimators, concentrating predominantly on the template method which is closer to the idea of SED fitting as discussed in the previous section.

\subsection{Methods}

\subsubsection{Empirical techniques}

Early on, the first empirical methods proved extremely powerful despite their simplicity (see e.g. Connolly et al. 1995a; Brunner et al. 1997; Wang et al. 1998). This was partly due to their construction, which should provide both accurate redshifts and realistic estimations of the redshift uncertainties. Even low-order polynomial and piecewise linear fitting functions do a reasonable job when tuned to reproduce the redshifts of galaxies (see e.g. Connolly et al.|1995a). These early methods provided superior redshift estimates in comparison to template-fitting for a number of reasons. By design the training sets are real galaxies, and thus suffer no uncertainties of having accurate templates. Similarly as the galaxies are a subsample of the survey, the method intrinsically includes the effects of the filter bandpasses and flux calibrations 
One of the main drawbacks of this method is that the redshift estimation is only accurate when the objects in the training set have the same observables as the sources in question. Thus this method becomes much more uncertain when used for objects at fainter magnitudes than the training set, as this may extrapolate the empirical calibrations in redshift or other properties. This also means that, in practice, every time a new catalogue is created, a corresponding training set needs to be compiled.

The other, connected, limitation is that the training set must be large enough that the necessary space in colours, magnitudes, galaxy types and redshifts is well covered. This is so that the calibrations and corresponding uncertainties are well known and only limited extrapolations beyond the observed locus in colour-magnitude space are necessary.

The simplest and earliest estimators were linear and polynomial fitting, where simple fits of the empirical training set in terms of colours and magnitudes with redshift were obtained (see e.g. Connolly et al. 1995a). These could then be matched to the full sample, giving directly the redshifts and their uncertainties for the galaxies. Since then further, more computational intensive algorithms, have been used, such as oblique decision tree classification, random forests, support vector machines, neural networks, Gaussian process regression, kernel regression and even many heuristic homebrew algorithms.

These algorithms all work on the idea of using the empirical training set to build up a full relationship between magnitudes and/or colours and the redshift. As each individual parameter (say the $B-V$ colour) will have some spread with redshift, these give distributions or probabilistic values for the redshift, narrowed with each additional parameter. This process, in terms of artificial neural networks, is nicely described by Collister and Lahav (2004), who use this method in their publicly available photo- $z$ code $\mathrm{ANNz}$ (described in the same paper). They also discuss the limitations and uncertainties that arise from this methodology.

Machine learning algorithms (of which neural networks is one) are one of the strengths of the empirical method. These methods are able to determine the magnitude/colour and redshift correlations to a surprising degree, can handle the increasingly large training sets (i.e. SDSS) and return strong probabilistic estimates (i.e. well constrained uncertainties, see figure 16) on the redshifts (see Ball et al.2008a for a description of machine learning algorithms available and the strong photo- $z$ constraints possible). In addition, machine learning algorithms are also able to handle the terascale datasets now available for photo- $z$ determination rapidly, limited only by processor speed and algorithm efficiency (Ball et al. 2008b).

The additional benefit of the empirical method with machine learning, now increasingly being used, is that the constraining inputs for the photo- $z$ s are not limited to the galaxies SED. Suggested first by Koo (1999), properties such as

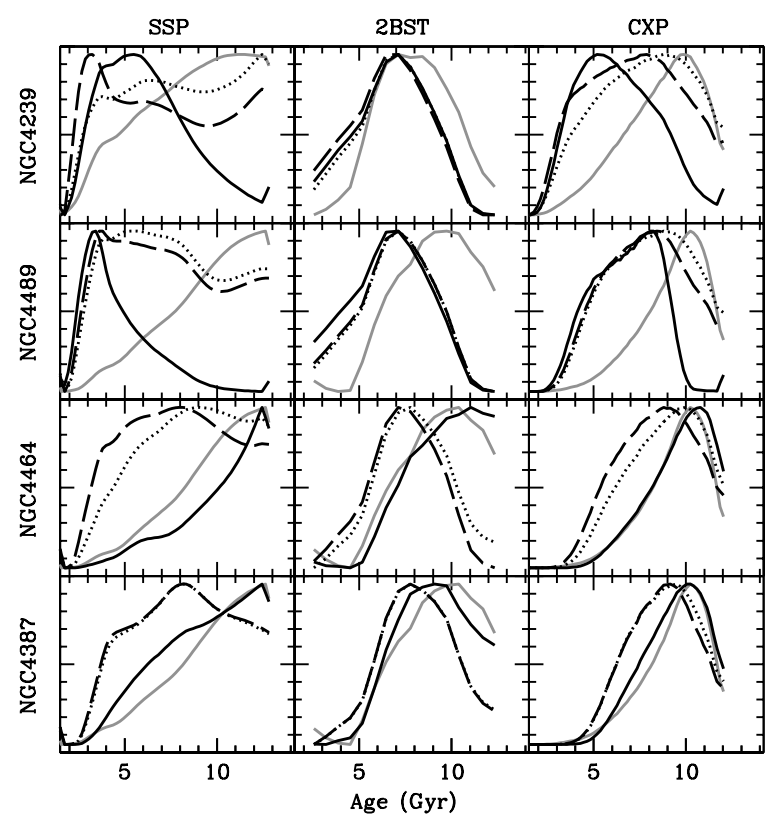

Fig. 15 Marginalized age distribution in four Virgo cluster elliptical galaxies. Three models for the star formation history are considered as labeled (see text for details). The black lines correspond to the age extracted from [MgFe] plus either $\mathrm{H} \beta$ (solid), $\mathrm{H} \gamma$ (dashed) or $\mathrm{H} \delta$ (dotted), respectively. The gray line is the result from the fit to the SED. [Courtesy I. Ferreras]
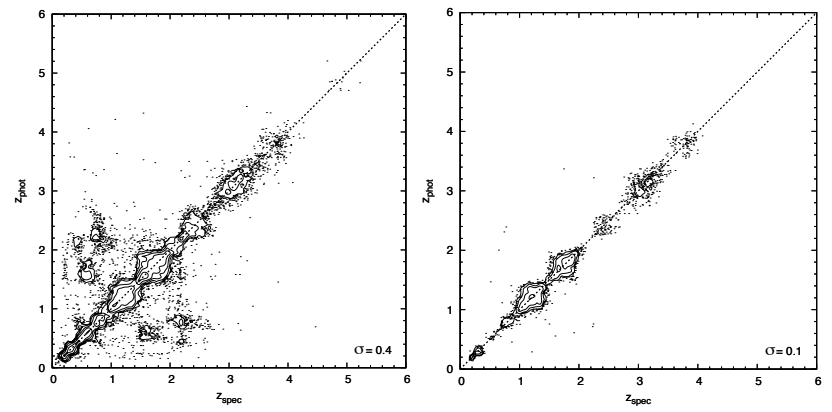

Fig. 16 Improvement in quasar redshifts enabled by the Ball et al. (2008b) data mining techniques, shown as spectroscopic versus photometric redshift for the SDSS. Left: A reproduction, using their framework, a typical result prior to their work (e.g. Weinstein et al. 2004) Right: The result of using machine learning to assign probability density functions then taking the subset with a single peak in probability. Contours indicate the areal density of individual quasars (points) on the plot. Data from Ball et al. (2008b). [Courtesy N.Ball] 
the the bulge-to-total flux ratio (e.g. Sarajedini et al. 1999), surface brightness (e.g. Kurtz et al. 2007), petrosian radii (e.g. Firth et al. 2003), and concentration index(e.g. Collister and Lahav 2004) have all been used in association with the magnitudes and colours to constrain the redshift, some codes even bringing many of these together (e.g. Wray and Gunn 2008).

\subsubsection{Template Fitting}

Unlike the empirical method, the template-based method is actually a form of SED fitting in the sense of this review (see e.g. Koo 1985, Lanzetta et al. 1996; Gwyn and Hartwick 1996, Pello et al.|1996; Sawicki et al. 1997). Put simply, this method involves building a library of observed (Coleman et al. 1980, is a commonly used set) and/or model templates (such as Bruzual and Charlot 2003) for many redshifts, and matching these to the observed SEDs to estimate the redshift. As the templates are "full" SEDs or spectra, extrapolation with the template fitting method is trivial, if potentially incorrect. Thus template models are preferred when exploring new regimes in a survey, or with new surveys without a complementary large spectroscopic calibration set. A major additional benefit of the template method, especially with the theoretical templates, is that obtaining additional information, besides the redshift, on the physical properties of the objects is a built-in part of the process (as discussed in section 4.1. Note though, that even purely empirical methods can predict some of these properties if a suitable training set is available (see e.g. Ball et al.|2004).

However, like empirical methods, template fitting suffers from several problems, the most important being mismatches between the templates and the galaxies of the survey. As discussed in section 2, model templates, while good, are not $100 \%$ accurate, and these template-galaxy colour mismatches can cause systematic errors in the redshift estimation. The model SEDs are also affected by modifiers that are not directly connected with the templates such as the contribution of emission lines, reddening due to dust, and also AGN, which require very different templates (see e.g. Polletta et al. 2007).

It is also important to make sure that the template set is complete, i.e. that the templates used represent all, or at least the majority, of the galaxies found in the survey (compare also Section 4.5.2. This is especially true when using empirical templates, as these are generally limited in number. Empirical templates are also often derived from local objects and may thus be intrinsically different from distant galaxies, which may be at different evolutionary stage. A large template set is also important to gauge problems with degeneracies, i.e. where the template library can give two different redshifts for the same input colours. Another potential disadvantage of template fitting methods comes from their sensitivity to many other measurements to about the percent level, e.g., the bandpass profiles and photometric calibrations of the survey.

For implementations of the template fitting, the method of maximum likelihood is predominant. This usually involves the comparison of the observed magnitudes with the magnitudes derived from the templates at different redshifts, finding the match that minimizes the $\chi^{2}$ (compare section 4.5). What is returned is the best-fitting (minimum $\chi^{2}$ ) redshift and template (or template+modifiers like dust attenuation). By itself this method does not give uncertainties in redshift, returning only the best fit. For estimations of the uncertainties in redshift, a typical process is to propagate through the photometric uncertainties, to determine what is the range of redshifts possible within these uncertainties. A good description of the template-fitting, maximum likelihood method can be found in the description of the publicly available photo- $z$ code, hyperz in Bolzonella et al. (2000).

As mentioned above, one of the issues of the templates is the possibility of template incompleteness, i.e. not having enough templates to describe the galaxies in the sample. Having too many galaxies in the template library on the other hand can lead to colour-redshift degeneracies. One way to overcome these issues is through Bayesian inference: the inclusion of our prior knowledge (see Section 4.5), such as the evolution of the upper age limit with redshift, or the expected shape of the redshift distributions, or the expected evolution of the galaxy type fractions. As described in Section 4.5, this has the added benefit of returning a probability distribution function, and hence an estimate of the uncertainties and degeneracies. In some respects, by expecting the template library to fit all observed galaxies in a survey, the template method itself is already Bayesian. Such methods are used in the BPZ code of Benítez (2000), who describes in this work the methodology of Bayesian inference with respect to photo- $z$, the use of priors and how this method is able to estimate the uncertainty of the resulting redshift.

It should be noted that, while public, prepackaged codes might provide reasonable estimates for certain types of sources, no analyses should proceed without crossvalidation and diagnostic plots. There are common problems that appear in data sets and issues that need to be understood first, and worked around, if possible (see e.g. Mandelbaum et al. 2005, for a comparison of some public photo- $z$ codes). Some further public photo- $z$ codes include kphotoz (Blanton et al.2003), zeBRA (Feldmann et al. 2006) and Le Phare (Arnouts et al. 1999, Ilbert et al. 2006, 2009a).

\subsection{Calibration and error budgets}

Redshift errors are ultimately data-driven: they typically scale with $1+z$ given constant wavelength resolution of most filter sets; they also scale with photometric error in a transition regime between $\sim 2 \%$ and $\sim 20 \%$. Smaller errors 
are often not exploited due to mismatches between data and model arising from data calibration and choice of templates, while large errors translate non-linearly into weak redshift constraints. If medium-band resolution is available, QSOs show strong emission lines and lead to deeper photo- $z$ completeness for QSOs than for galaxies.

Photometric redshifts have limitations they share with spectroscopic ones, and some that are unique to them: as in spectroscopy, catastrophic outliers can result from the confusion of features, and completeness depends on SED type and magnitude. Two characteristic photo- $z$ problems are mean biases in the redshift estimation and large and/or badly determined scatter in the redshift errors. Catastrophic outliers result from ambiguities in colour space: these are either apparent in the model and allow flagging objects as uncertain, or are not visible in the model but present in reality, in which case the large error is inevitable even for unflagged sources. Empirical models may be too small to show local ambiguities with large density ratios, and template models may lack some SEDs present in the real Universe.

Remedies to these issues include adding more discriminating data, improving the match between data and models as well as the model priors, and taking care with measuring the photometry and its errors correctly in the first place. Photo- $z$ errors in broad-band surveys appear limited to a redshift resolution near $0.02 \times(1+z)$, a result of limited spectral resolution and intrinsic variety in spectral properties. Tracing features with higher resolution increases redshift accuracy all the way to actual spectroscopy. Future work among photo- $z$ developers will likely focus on two areas: (i) Understanding the diversity of codes and refining their performance; and (ii) Describing photo- $z$ issues quantitatively such that requirements on performance and scientific value can be translated into requirements for photometric data, for the properties of the models and for the output of the codes.

\subsubsection{Template accuracy}

In general, template-based photo- $z$ estimates depend sensitively on the set of templates in use. In particular, it has been found that better photo- $z$ estimates can be achieved with an empirical set of templates (e.g. Coleman et al. 1980, Kinney et al. 1996) rather than using stellar population synthesis models (SPSs; e.g. Bruzual and Charlot 2003; Maraston 2005, see Section 2) directly. Yet the models are what are commonly used to compute stellar masses of galaxies. Since the use of these templates do not result in very good photometric redshifts, what is usually done, is to first derive photometric redshifts through empirical templates, and then estimate the stellar masses with the SSP templates. Obviously this is not self-consistent.

To investigate what causes the poorer photo- $z$ estimates of synthetic templates, Oesch et al. (in prep.) used the photometric data in 11 bands of the COSMOS survey (Scoville et al. 2007), together with redshifts of the zCOSMOS follow-up (Lilly et al. 2007) and fit the data with SSP templates. In the resulting rest-frame residuals they identified a remarkable feature around $3500 \AA$, where the templates are too faint with respect to the photometric data, which can be seen in figure 17 The feature does not seem to be caused by nebular continuum or line emission, which they subsequently added to the original SSP templates. Additionally, all types of galaxies suffer from the same problem, independent of their star-formation rate, mass, age, or dust content.

Similar discrepancies have been found previously by Wild et al. (2007); Walcher et al. (2008), who found a $\sim 0.1$ mag offset in the $\mathrm{D}_{\mathrm{n}}(4000)$ index. As this spectral break is one of the main features in the spectrum of any galaxy, it is likely that the poor photo- $z$ performance of synthetic templates is caused by this discrepancy. The cause of the discrepancy has been identified as a lack of coverage in the synthetic stellar libraries used for the models. It will thus be remedied in the next version of GALAXEV (G. Bruzual, priv. comm.).

\subsubsection{Spectroscopic Calibration of Photo-zs}

One of the strong benefits of the template method is that any spectroscopic subsample of a survey can be used to check the template-determined photo-zs. This can also be done for the empirical methods, yet for this a very large spectroscopic sample is necessary such that it can be divided into a large enough training set and testing sets.

With the existence of a test spectroscopic sample, it is then possible to calibrate the template library, leading to a combined empirical-template method. This means to correct for errors in the photometric calibration or even the correction of the templates themselves for example to allow for the evolution of galaxies with a small library, or to account for inaccurate models (see section 2). Such calibration is typically an iterative process, in which the photometry and/or template SEDs are modified to minimize the dispersion in the resulting photometric redshifts.

The simplest kind of calibration involves adding small zero-point offsets to the photometry uniformly across the sample. This does not imply that the photometry is incorrectly calibrated (though in practice the absolute calibration may well have small errors in the zero-point), but rather that there is often a mismatch between the real SEDs of galaxies and the templates used to fit them. The calibration is meant to minimize those differences. Plotting color-color or color-redshift diagrams (figure 18) with the template SEDs overlaid will often indicate bulk offsets between the two.

A more instructive approach, however, is to compute the residuals between the predicted magnitude of the best-fit template at the spectroscopic redshift and the observed magnitude (for more details, see Brodwin et al. 2006a b). These residuals can be plotted versus color or redshift for added 
diagnostic power. In the example in Figure 19 , there appears to be an effective magnitude offset of $\approx 0.3$ mags in the $H$-band.

Applying such effective zero-point adjustments in all bands in an iterative process minimizes the mismatch between the data and the templates, and hence minimizes the resultant photometric redshift dispersion, as shown in Fig. 20 .

Such calibration phases are used in the works of Brodwin et al. (2006a) and as "template-optimization" in the codes ZEBRA (Feldmann et al. 2006) and Le Phare (Ilbert et al. 2006. 2009a) which use template fitting with Bayesian inferences and this calibration phase to give the most accurate photometric redshifts possible with the template approach.

With the most accurate photometric redshifts possible, the template-fitting can then be used to estimate physical properties such as stellar masses, star-formation rates, etc. (see section 6).

\subsubsection{Signal-to-noise Effects}

Margoniner and Wittman (2008) have specifically investigated the impact of photometric signal-to-noise ( $\mathrm{SN}$ ) on the precision of photometric redshifts in multi-band imaging surveys. Using simulations of galaxy surveys with redshift distributions (peaking at $z \sim 0.6$ ) that mimics what is expected for a deep (10-sigma $R$ band $=24.5$ magnitudes) imaging survey such as the Deep Lens Survey (Wittman et al. 2002) they investigate the effect of degrading the SN on the photometric redshifts determined by several publicly available codes (ANNz, BPZ, hyperz)

Figure 21 shows the results of one set of their simulations for which they degraded the initially perfect photometry to successively lower SN. In these unrealistic simulations all galaxies have the same $\mathrm{SN}$ in all bands. The figure shows the cumulative fraction of objects with $\delta_{z}$ smaller than a given value as a function of $\delta_{z}$. The left panel shows the cumulative fraction for all objects, while the right panel shows galaxies for which the BPZ photo- $z$ quality parameter, $O D D S>0.9$. The number of galaxies in the right panel becomes successively smaller than the number in the left as the signal-to-noise decreases ( $64 \%$ of $\mathrm{SN}=250$, and only $6.4 \%$ of $\mathrm{SN}=10$ objects have $O D D S>0.9$ ), but the accuracy of photo-zs is clearly better.

The results of this work show (1) the need to include realistic photometric errors when forecasting photo-zs performance; (2) that estimating photo-zs performance from higher SN spectroscopic objects will lead to overly optimistic results.

\subsection{A unified framework}

The field of photometric redshifts and the estimation of other physical properties has been very pragmatic. Its development, since the first attempts, has been incremental in the sense that most studies focused on refining components but staying within the concepts of the original ideas of the two classes. Empirical and template-fitting approaches today follow very separate routes, with these classes of methods even use different sets of measurements. Only the semiempirical approach of zero-point calibration comes close to linking the two approaches. However, a recent study by Budavári (2009a), tries to understand this separation and possibly bring these methods together by devising a unified framework for a rigorous solution based on first principles and Bayesian statistics.

This work starts with a minimal set of requirements: a training set with some photometric observables $\boldsymbol{x}$ and spectroscopic measurements $\xi$, and a query or test set with some potentially different set of observables $\boldsymbol{y}$. The link between these is a model $M$ that provides the mapping between $\boldsymbol{x}$ and $\boldsymbol{y}$, the probability density $p(\boldsymbol{x} \mid \boldsymbol{y}, M)$. This is more than just the usual conversion formula between photometric systems because it also incorporates the uncertainties.

The empirical relation of $\boldsymbol{x}-\boldsymbol{\xi}$ is often assumed to be a function. A better approach is to leave it general by measuring the conditional density function. The simplest way is to estimate the relation by the densities on the training set as $p(\boldsymbol{\xi} \mid \boldsymbol{x})=p(\boldsymbol{\xi}, \boldsymbol{x}) / p(\boldsymbol{x})$. The final result is just a convolution of the mapping and the measured relation: $p(\boldsymbol{\xi} \mid \boldsymbol{y}, M)=\int d \boldsymbol{x} p(\boldsymbol{\xi} \mid \boldsymbol{x}) p(\boldsymbol{x} \mid \boldsymbol{y}, M)$. In figure 22 we show the results from Budavári (2009a), where he plots the empirical relation (dotted blue line) and the final probability density (solid red) for a handful of SDSS galaxies. The top panels show intrinsically red galaxies, whose constraints are reasonably tight out to the highest redshifts. Blue galaxies in the bottom panels however get worse with the distance as expected.

The aforementioned application follows a minimalist empirical approach but already goes beyond traditional methods. Template-fitting is in the other extreme of the framework where the training set is generated from the model using some grid. Without errors on the templates, the equations reduce to the usual maximum likelihood estimation that is currently used by most codes. A straight forward extension Budavári (2009a) suggests is to include more realistic errors for the templates. Similarly one can develop more sophisticated predictors that leverage existing training sets and spectrum models at the same time.

\subsection{The State of Photometric Redshifts}

Generally there is an overall agreement in most aspects of photometric redshift methodologies, and even technicalities. However there is a need for standardized quality measures and testing procedures. It is important to analyze to performance of each model spectrum as a function of the redshift. This is best done by plotting the difference of the observed 
magnitudes and template-based ones. These figure can pinpoint problems with the spectra and even zero-points. Intrinsically these are the quantities used inside the templateoptimization procedures, e.g., in Budavári et al. (2000) and Feldmann et al. (2006).

In SED fitting, interpolation between templates is often used, which can be done linearly or logarithmically. The latter has the advantage of being independent of the normalization of the spectra. Yet, most codes appear to use linear interpolation without a careful normalization. This might explain some of the discrepancies among similar codes found by the Photo- $Z$ Accuracy Testing (PHAT) project. 5

The determination of the quality of the estimates is also a crucial topic. There is need for different measures that can describe the scatter of the points without being dominated by outliers and that can estimate the fraction of catastrophic failures. It is also recommended to characterize the accuracy of the estimates by a robust M-scale instead of the RMS; a measure that is simple to calculate, yet, not sensitive to outliers. Another aspect of this is the study of selection criteria that is often neglected. Certain projects are not concerned with incomplete samples as long as the precision of the ones provided is good (e.g., weak lensing, Mandelbaum et al. 2005), while others, such as galaxy clustering, might require an unbiased selection. Therefore, it is perceived that studies using methods with any quality flags or quantities should provide details of their selection effects.

A common theme for future goals in most photometric redshift works appears to be more detailed probabilistic analyses, with the need for probability density functions. Priors used in most bayesian analyses seem to be generally accepted in the photo- $z$ community. With such consensus amongst photometric redshifts obtained, the focus of work now is shifting from the estimation of "just" the redshifts to simultaneously constraining physical parameters and the redshift in a consistent way.

\section{Results of SED Fitting: Physical Properties}

SED fitting is a very versatile tool. From a rough estimation of the stellar masses of distant galaxies to the search for small subpopulations of stars in high $\mathrm{S} / \mathrm{N}$ spectra, it can be applied to a large part of the problems in galaxy evolution. This is the strength and the weakness of the SED fitting technique: it does it all at once.

We highlight here a few significant results. The intention is not to be complete or to mention the work that has been most in view, but rather to highlight the diversity of questions that can be adressed from fitting the integrated SEDs of

5 http://www.astro.caltech.edu/twiki_phat/bin/view/Main/ WebHome stellar populations. Particular importance has been given to supply cautionary remarks, as it is easy to overinterpret the significance of the derived properties, in view of the complexity of the physical mechanisms and our frequent lack of detailed understanding.

\subsection{Stars}

\subsubsection{Stellar masses}

Stellar masses are computed by multiplying a mass-to-light ratio $M / L$ with a luminosity $L$. While the uncertainties on $L$ depend on the quality of the data, the estimate over $M / L$ and its associated uncertainties depend mostly on the care taken with SED fitting. It is a good idea to search for a reference band that minimizes the effects of $M / L$ variations due to stellar populations (age, metallicity, chemical abundances) and due to dust absorption. While the common notion that the NIR (e.g. the $H$ band at $1.65 \mu \mathrm{m}$ ) is close to ideal is correct in some cases, problems can arise because of thermally pulsing asymptotic giant branch stars (discussed in section 2.1.3) if young ages $(<2 \mathrm{Gyr})$ are well represented in the SED. That reliable $M / L$ from SED fitting cannot be dispensed with is evident when looking at IRAC $3.6 \mu \mathrm{m}$ data of nearby galaxies, where star formation regions are evidently prominent.

Stellar Mass Maps of Resolved Galaxies In the work of Zibetti et al. (2009) a "data-cube" approach is introduced to investigate the SEDs of nearby, resolved galaxies, aimed at preserving the maximum spatially-resolved information. One feature of the approach is that it allows to compare "global" quantities, which are notoriously difficult to determine, with the integral over the local quantities, a useful test of how meaningful global quantities can be. A large part of the effort concentrated on developing a reliable method to obtain stellar surface mass density maps from a minimum set of broad-band observations. This method relies on Bayesian inference, as discussed in Section 4.5 .

The effective mass-to-light ratio correlates with optical / near-infrared colors (e.g., Bell and de Jong 2001), so $M / L$ can be expressed as a function of color(s). A better estimate is obtained if $M / L$ is mapped as a function of two colors, instead of one. The colors adopted are $g-i$ and $i-H$. Their large wavelength separation allows to robustly describe the shape of the SED over the entire optical-near-infrared range, in a way that as insensitive as possible to photometric and modeling uncertainties.

To study the dependence of $M / L$ on $(g-i, i-H)$ the authors use a Monte Carlo library of 50,000 models created from the 2007 version of $\mathrm{BC} 03$, which include also dust in different amounts and spatial distributions.. The $(g-i, i-H)$ space is binned in cells of $0.05 \mathrm{mag} \times 0.05 \mathrm{mag}$ 
and marginalized over $M / L$ in each cell. The median is chosen as the fiducial $M / L$ at each position of the color-color space. A look-up table is created to derive $M / L_{H}$ as a function of $(g-i, i-H)$. Figure 23 illustrates that the information in the second color improves the $\mathrm{M} / \mathrm{L}$ determinations systematically by $\pm 0.3-0.4$ dex.

The method described above is applied to each pixel of the image of a galaxy, where "pixel" implies the pixel that results after matching the images in the three bands to a common resolution. In order to provide sensible results, it is crucial that color measurements do not exceed $0.1 \mathrm{mag}$ errors, which requires $\mathrm{S} / \mathrm{N} \sim 20$. The results for $\mathrm{M} 100$ (NGC 4321) are displayed in Figure 24

An important result appears from the comparison between total stellar mass as obtained by integrating the stellar mass surface density maps (Figure 24d) and the one obtained using global colors to estimate the "average" $M / L$ ratio to be multiplied by $L(H)$. This second method, the one commonly adopted in extragalactic studies, agrees within $\sim 10 \%$ of the mass map integral only when the color distribution is quite homogeneous, i.e., for early type galaxies. When substantial color inhomogeneities and especially heavily obscured regions are present within a galaxy, using global colors and fluxes can lead to underestimates of the actual stellar mass of a galaxy by up to $60 \%$. This can be understood if dust-obscured regions can contribute a significant amount of mass but are heavily under-represented in the global color, which is flux weighted and hence biased toward the brightest (and bluest) parts of the galaxy. While the pixel-by-pixel $M / L$ gives the correct mass weight to these obscured regions, the globally computed $M / L$ severely underestimates their mass contribution.

Stellar mass functions One of the holy grails of current observational efforts in galaxy evolution studies is a consistent picture of the build-up of stellar mass over the age of the universe. An important constraint on this is the stellar mass function, or its integral, the stellar mass density. A comprehensive discussion of these results would warrant a review of its own. Suffice it here to point out that not only the local mass function has been measured with great precision (e.g. Bell et al.|2003a), but these results have also been extended to redshifts of 1 (Pozzetti et al. 2007, Bundy et al. 2006). At redshifts above 1.2 an observed-frame optical selection corresponds to a rest-frame UV selection, subject to large biases. These therefore have to be circumvented by a K-band selection (e.g. Cirasuolo et al. 2007) or, better, by a selection at 3.6 $\mu \mathrm{m}$ (Arnouts et al. 2007, Ilbert et al. 2010. e.g.). For observational and conceptual reasons (detailed in Sections 4.5.2 and 4.6, determining stellar masses and therefore mass function at redshifts higher than $\mathrm{z}=2$ is very difficult. Most authors thus prefer to restrict themselves to luminosity functions instead (see e.g. Cirasuolo et al. 2010. for just one very recent example), thus leaving the burden of transforming luminosities to stellar masses to the interpreting model (but see e.g. Kajisawa et al.2009).

Stellar masses of high redshift galaxies Many authors have used some kind of Bayesian inference-based method (Section 4.5 to determine stellar masses for high redshift galaxies (e.g. Sawicki and Yee 1998: Papovich et al. 2001; Förster Schreiber et al.2004). There is good hope that these mass estimates are reasonably good (Drory et al. 2004), despite important caveats on the methodology that become more important with improving data quality (see e.g. Section 4.1). A recent result has been the discovery and study of high redshift galaxies with high stellar masses and low star formation rates (early type galaxies, ETGs). In the following we describe only one "family" of papers, as presented at the workshop, but see Cimatti (2009) for a review. Massive ETGs are the first objects to populate the red sequence (see e.g. Kriek et al. 2008b). Objects in the redshift range 2-3 can be identified by multi-band photometry (e.g. van Dokkum et al. 2006). For determining the physical properties however, significant uncertainties are due to photometric redshift determinations. For example Kriek et al. (2008) conclude that while stellar masses are reasonably robust to small errors arising from photometric redshifts, the actual star formation history is generally very poorly constrained with broad band data alone.

The obvious next step is thus to analyze these galaxies with spectroscopy, despite this being an expensive undertaking in terms of telescope time. When doing this, Kriek et al. (2008) also go further in blurring the limits between spectroscopy and photometry by binning their "low" $\mathrm{S} / \mathrm{N}$ spectra into bins of $400 \AA$. While the information content remains unchanged, this certainly leads to improvements in presentability and fitting speed. Kriek et al. (2009a) then show explicitly that provided enough photons can be assembled through either exposure time or telescope size, the spectra of galaxies at redshifts 2-3 are amenable to the same kind of analysis as in the local universe. The upshot of these studies is that massive, compact ETGs with very little residual star formation are in place already at redshifts between 2 and 3.

Despite these successes, the study of Muzzin et al. (2009a|b) confirms that even using spectroscopic data, model uncertainties mean that SED-derived stellar masses are affected with uncertainties of factors 2-3 at these redshifts. For further discussion on stellar mass determinations the reader is also referred to the review by de Jong \& Bell (in prep.).

\subsubsection{Deriving SFHs from spectroscopy}

Comparing observations to semi-analytic models Trager and Somerville (2009) extend the semi-analytic model of 
Somerville et al. (2008) to predict the line absorption strengths (Section 4.2) of the resulting galaxies. This allows them to use the same analysis tools that would be used in the analysis of the measured line strengths of an observed sample of galaxies on objects with known properties, in particular star formation histories. They select in particular early type galaxies from the mock catalogues they produce and compute the index strengths of the resulting spectra. These index strengths can then be plotted on the same plots as real data. They come to the sobering conclusion that while the sample of Trager et al. (2008) is of sufficient quality do do a meaningful comparison, it remains too small. On the other hand large samples of galaxies, as the one of Moore et al. (2002), still lack the required precision.

The archeology of the universe The database of the SDSS spectra has been used to derive the SFH of galaxies from their current spectra (e.g. Heavens et al. 2004, see also Section 4.4, a procedure sometimes called "unlocking the fossil record" or simply "astro-archeology". A recent update on this has been presented in Tojeiro et al. (2007), who applied VESPA (see Section 4.4) to the SDSS sample of spectra and derived a catalogue that was made available to the community at http://www-wfau.roe.ac.uk/vespa/ It provides detailed star formation histories and other parameters for SDSS's latest data release (DR7) of the Main Galaxy Sample and the Luminous Red Galaxies sample. Details of the catalogue, including description, basic properties and example queries can be found in (Tojeiro et al.|2009).

Combining spectroscopic and dynamic ages The use of spectroscopy in combination with dynamical arguments to understand the evolution of a single object was presented in Pappalardo et al. (2010). The galaxy NGC4388 is a member of the Virgo cluster and sports a huge trail of HI gas (Oosterloo and van Gorkom 2005). It represent an ideal study case for the effects of ram stripping on a galaxy moving in the intra-cluster medium (Vollmer and Huchtmeier 2003). The stripping age has been estimated to be of order 200 Myr from dynamical arguments. Using VLT/ FORS spectroscopy of the outer and inner regions of the galaxy and the STECKMAP program (Ocvirk et al. 2006), Pappalardo et al. (2010) were able to show that, while the inner region of the galaxy is of solar metallicity and has continued forming stars to the present day, the outer regions of the galaxy have sub-solar metallicity and have stopped forming stars roughly 200 Myrs ago, in accordance with the dynamical estimate. Single cases like this can thus help identify the processes and timescale associated with shutting down the star formation in galaxies, one of the most profound changes a galaxy can experience.

Star formation rates Star formation rates from SED fitting have been little used, with most authors preferring to rely on single tracers (see Section 4.5.3 for a comparison). Walcher et al. (2008) have used stellar masses and star formation rates consistently derived from the same SED fit to compare the predicted evolution of the stellar mass function to the observed one. The main result is that while stars form in blue cloud galaxies, most of the growth of the stellar mass function occurs in quiescent galaxies, in agreement with studies based on different tracers of star formation (e.g. Bell et al. 2007). From comparison with merger studies in the same field, Walcher et al. (2008) conclude that about half of the mass growth on the red sequence comes from major mergers and half from minor mergers.

Salim et al. (2007) have compared their SED-fitting SFRs to SFRs determined from emission lines. They find that some galaxies with no detected emission lines nevertheless have substantial SED-based SFRs. They attribute this result to "recent" star formation, i.e. stars that formed long enough ago that their emission lines already vanished, but still recently enough to be revealed in the galaxy SED. Recent HST imaging in the UV which clearly shows SF structures seems to confirm this (Salim \& Rich 2010, ApJ submitted).

\subsubsection{Identifying and studying outliers}

This is an underexplored use of SED fitting, in the opinion of the authors. One example, objects with differing SFR measurements from emission lines and SED fitting has been covered in the last section.

Finding Wolf-Rayet stars The availability of large databases of spectra, such as from the SDSS, and of accurate stellar population model predictions enables the search for rare objects or systematic deviations that are not predicted by the model itself. An example of this are Wolf-Rayet stars, evolved, massive stars with characteristic features. These have ages between $2 \times 10^{6}$ and $5 \times 10^{6}$ years, and are thus a transient feature of galaxy spectra. They are useful as a tracer of recent star formation history as well as the metallicity of their host galaxies. As shown in Brinchmann et al. (2008), a systematic search in the SDSS database yields a sample several times larger than previous serendipitous searches. The essential ingredient of such a search is the accuracy of the stellar population model that allows an inversion technique (Section 4.4) to be applied on a large sample. Indeed, either a smooth correction or residual features from inaccurate models would severely impair the identification of the specific features. As an example Brinchmann et al. (2008) show that the use of the Bruzual and Charlot (2003) models produced a large number of false positives, while an updated version of the same model using different stellar spectra (CB09) provides much better fits. 


\subsection{Dust}

Dust cannot be ignored when fitting a galaxy's SED, as shown by the cosmic infrared background, which has comparable power to the distinct peak of the cosmic UV-optical background (Hauser and Dwek|2001). The relative strength of the cosmic background in the infrared suggests a significant processing of the galactic stellar light over the age of the universe. This processing must have also been more significant with increasing redshift as the percentage of stellar light re-radiated by dust is only $\sim 30 \%$ locally (Popescu and Tuffs 2002), as supported by the increasing number density of luminous IR galaxies up to $z \sim 1.3$ (Magnelli et al. 2009).

As discussed in section 2 the absorption and emission of light by dust are generally treated as separate processes in modelling, and this is similarly true in SED fitting.

\subsubsection{Attenuation by Dust}

Dust between the observer and the individual stars of a galaxy acts to extinguish and redden the light from those stars. When the stars in our own Galaxy were examined it was found that a simple relation with wavelength was able to describe the extinction and reddening by dust for a wide range of galactic environments, with the only strong feature occurring at $\sim 2175 \AA$ (Cardelli et al. 1989). A similar but steeper extinction law was found for the Magellanic Clouds, with weaker or non-existent feature at $2175 \AA$ (Gordon and Clayton 1998; Misselt et al. 1999). It is these extinction laws that have given rise to the contemporary model of dust in the ISM (i.e. Mathis et al. 1977), and the understanding that the dust composition between the Milky Way and Magellanic clouds is different.

Yet when integrated over the whole of a galaxy the situation becomes complex, with the geometry of the stars and dust strongly affecting the resulting spectrum. The effects of varying amounts of extinction of the different stellar populations due to the spatial distribution of stars and clumpy dust, and the scattering of blue stellar light into our line of sight act to flatten the effects of dust on the spectrum, creating an attenuation law, where the amount of reddening with extinction is less (or 'greyer') than we observe locally (Witt et al. 1992). This was exactly what was found in starburst galaxies by Calzetti et al. (1994), and Charlot and Fall (2000) found that a simple screen effective attenuation (i.e. a screen of dust between the galaxy and observer) with a power-law relation, $\tau_{\text {ISM }} \propto \lambda^{-0.7}$, was able to account reasonably well for the diffuse ISM attenuation in galaxy observations. It is this complexity that makes disentangling the effects of geometry and differing dust difficult, and thus the extraction of physical dust properties from galaxy SEDs problematic. There are two areas where some progress has been made.
The $2175 \AA$ feature The $2175 \AA$ feature has been associated with small carbonaceous grains in the ISM (Mathis et al. 1977), and is observed in both the Milky way, M33 (Gordon et al. 1999), and (weakly) in the LMC, but is almost non-existent in the SMC. This feature is not observed in the attenuation law of starburst galaxies (Calzetti et al. 1994). Whether this lack is due to the clumpy geometry of dust and stars (Fischera et al. 2003) or is actually indicative of SMClike dust in starburst galaxies (Gordon et al. 1999) is still under debate, yet this feature is generally not needed to fit the attenuation of galaxies. In QSOs, which, being dominated by a nuclear source, are closer to the galactic extinction situation, an average attenuation curve does not show this feature, suggesting processing of the ISM in these active objects (Czerny et al. 2004). However, in a few non-local galaxies where direct extinction lines of sight are available, this feature has been observed, suggesting it may be more common than the attenuation curves of local galaxies suggest (Wang et al. 2004; Elíasdóttir et al. 2009). At higher redshifts, where UV spectra are more commonly observed, recent studies find evidence for the existence of the $2175 \AA$ bump (e.g. Noll et al. 2007;, Noterdaeme et al. 2009).

Young versus old attenuation One important progress made in the treatment of galaxy attenuation is the realisation that the effective attenuation of a galaxy is dependent upon its star formation history. Calzetti (1997) found that in starburst galaxies the effective attenuation of the stellar continuum was less than that suffered by the nebula emission, as measured through emission lines. This clear indication of the clumpiness of the dust in galaxies has been interpreted as an indication of differential attenuation of different stellar populations, with young stars, and their associated ionized nebula, strongly attenuated by the clouds from which the stars formed, while older stars have evolved out of their 'birth clouds' either through cloud or stellar dispersion, and are only attenuated by the diffuse ISM dust, which acts on both the young and old stars (see e.g. Charlot and Fall 2000). Exactly what is the clearing time of these clouds and the differential attenuation is still uncertain, and may be galaxy specific, but this forms a basis for current galaxy SED models as discussed in section 2 .

\subsubsection{Dust Emission}

Extracting physical properties from dust emission in the IR is difficult for both theoretical and observational reasons: excluding the mid-IR, there are no observed dust features, most being washed out due to the broad shape of the blackbody emission; the IR suffers from strong observational constraints, with most data coming from space- and balloonbased observations; associated with this is the, until recently, limited sensitivity and spatial resolution and in the far-IR, at wavelengths $>100 \mu \mathrm{m}$, the sparsity of data. 
With ISO and, especially, Spitzer space telescopes this situation has recently improved, and will improve more so in the near future with the recent launch of Herschel and ALMA beginning to take form. So we touch upon here some of the galaxy physical properties that have been determined from the dust IR emission.

PAH emission in the Mid-IR As mentioned in section 2 , the $5-20 \mu \mathrm{m}$ mid-infrared spectrum of galaxies is generally dominated by broad emission features arising from large molecules, polycyclic aromatic hydrocarbons (see e.g. Smith et al. 2007). Underlying these features is the stellar continuum at short wavelengths and hot dust emission. Confusing the interpretation of the emission features are strong ionic emission lines arising from species such as $\mathrm{Ne}^{+}$and $\mathrm{Ne}^{++}$and strong, broad absorption features from silicate grains at $9.8 \mu \mathrm{m}$ and $18 \mu \mathrm{m}$.

A recent tool, PAHFIT, has been developed to decompose the mid-infrared spectra into its stellar, PAH, dust continuum, and line emission constituents, using functional forms and templates for the features in this wavelength range (Smith et al.2007). An example of this can be seen in figure 25 . The PAH feature luminosity has been used as star formation rate tracers (see section 6.2.4), and the relative strength of these features to the continuum have been found to be strongly linked to the presence of AGN (see e.g. Spoon et al. 2007, and below), and to the gas phase metallicity (see e.g. Smith et al. 2007). The relative strengths of these features can also be used to diagnose the mean size and ionization state of the PAHs, which is related to the average radiation field and dust size distribution (Draine and Li 2007a).

Diagnosing the energy source in ULIRGs Due to the high obscuration by dust in IR bright galaxies, especially ultra luminous IR galaxies (ULIRGs), diagnosing the dominant heating source is problematic. The diagram put forward by Spoon et al. (2007) helps resolve this issue by using the strength of the strong silicate absorption feature that is determined from fitting the mid-IR SED (as discussed above in Section 6.2.2 in association with the equivalent width of the PAH features. This diagram cleanly separates different classes of ULIRGs, from obvious Seyfert galaxies, strongly starbursting galaxies, and to deeply buried AGN ULIRGs and represents one of the strengths of IR SED fitting, extracting information from objects which are heavily obscured at shorter wavelengths.

Dust masses One of the more important properties obtained by fitting the IR SED is the dust mass. Through fitting of the far-IR SED the temperature(s) and the relative contributions of the different dust components that make up the SED can be constrained. Then, using knowledge of the emissivity per unit mass of dust, the total dust mass $\left(M_{\mathrm{d}}\right)$ can be determined, using an equation such as (based on Dunne and Eales 2001);

$M_{\mathrm{d}}=\frac{L_{850}}{\kappa_{\mathrm{d}}(850)}\left[\sum_{k} \frac{N_{k}}{B\left(850, T_{k}\right)}\right]$,

with $L_{850}$ the $850 \mu \mathrm{m}$ luminosity, and $N_{k}$ and $T_{k}$ the relative contribution and temperature of dust component $k$. The sum of dust components is usually limited $(\leq 3)$ by the sparse observational points at long wavelengths, but can also be represented by an integral of temperatures, parametrized by the strength of the heating radiation field (such as used by, e.g. Dale and Helou 2002, Draine and Li 2007a). $\kappa_{\mathrm{d}}(850)$ is the dust mass opacity coefficient, taken to be $0.077 \mathrm{~m}^{2} \mathrm{~kg}^{-1}$ by Dunne et al. (2000); Dunne and Eales (2001), an intermediate value between graphite and silicate. It is generally with this parameter that most of the uncertainties in determining dust masses remain.

Longer wavelength fluxes ( $>300 \mu \mathrm{m}$, such as $850 \mu \mathrm{m}$ ) are preferable to shorter wavelengths when determining dust masses as these sample the Rayleigh-Jeans part of the Planck curve, where the flux is least sensitive to temperature. Longer wavelengths are also more sensitive to the mass of the emitting material, as they are sensitive to cold dust as well as warm.

Clear examples of fitting the far IR SED using simple, emissivity-modified black-bodies and determining the total dust masses can be found in Dunne et al. (2000). Dunne and Eales (2001) and more recently in Clements et al. (2009) (see also da Cunha et al. 2010, Section 6.3 below). These works detail nicely the pertinent issues with both the data and fitting the far-IR SEDs. One of the best examples of determining the total dust mass, as well as other parameters, using the full IR SED was done by Draine et al. (2007b). Their physically based SED models (described in detail in Draine et al. 2007b) were fitted to the far-IR SEDs of galaxies from the SINGS sample, and gave determinations of the total dust mass, PAH fraction and information on the interstellar radiation field heating the dust. They found that dust in spiral galaxies resembled that found in the local Milky Way ISM, with similar dust-to-gas ratios, and that generally it is the diffuse ISM that dominates the total IR power, excluding strong starbursting systems. These results thus confirmed the earlier ISO discoveries (see the review by Sauvage et al. 2005). Note also that even earlier detailed radiative transfer modelling of individual galaxies had pointed to the dominance of the diffuse component (Popescu et al. 2000).

Sub-mm excess emission SED fitting can not only return physical properties, but can also indicate where our current knowledge is failing. As mentioned above, the long wavelength dust emission is a good handle for the total dust mass. 
However, when fitting the IR SED of several dwarf galaxies it has been found that the sub-mm flux is in excess to a standard cool dust-body emission, requiring additional dust components at a unreasonably low temperatures $(\lesssim 7 \mathrm{~K})$ to fit the SED (Lisenfeld et al. 2002; Israel et al. 2010, e.g.). While very cold large grains could be one possible cause, other suggestions have included small stochastic grains that spend most of their time at cold temperatures (Lisenfeld et al. 2002), rotating dust grains (Israel et al.2010), or some modification of the dust emissivity at these wavelengths or temperatures (Draine and Lee 1984: Weingartner and Draine 2001). Either way until this issue is resolved on the cause of this excess, the dust mass of these dwarf galaxies such as NGC 1569 will have large uncertainties. It is hoped that telescopes such as Herschel and ALMA may find more of these objects in the near future and help find the cause of this excess emission.

\subsubsection{Dust in the UV to IR}

The infrared-to-ultraviolet ratio is a coarse measure of dust extinction in the ultraviolet, and thus should be related to the amount of reddening in ultraviolet spectra. Indeed, starbursting galaxies follow a tight correlation between the ratio of infrared-to-ultraviolet emission and the ultraviolet spectral slope (e.g. Calzetti 1997;, Meurer et al.|1999). Compared to the relation defined by starbursts, normal star-forming galaxies are offset to redder ultraviolet spectral slopes, exhibit lower infrared-to-ultraviolet ratios, and show significantly larger scatter (Kong et al. 2004, Buat et al. 2005. Burgarella et al. 2005; Seibert et al. 2005; Cortese et al. 2006: Boissier et al. 2007; Gil de Paz et al.|2007; Dale et al. 2007). Offsets from the locus formed by starbursting and normal star-forming galaxies can be particularly pronounced for systems lacking significant current star formation, such as elliptical galaxies, systems for which the luminosity is more dominated by a passively evolving older, redder stellar population.

Using a sample of 1000 galaxies with spectroscopy from the SDSS and homogeneous photometric coverage from the UV to $24 \mu \mathrm{m}$ from SDSS and the Galex and Spitzer satellites, Johnson et al. (2007a) found that the sample galaxies span a plane in the three-dimensional space of NUV$3.6 \mu \mathrm{m}$ colour, $\mathrm{D}_{n}(4000)$ index (as defined by Balogh et al. 1999), and infrared excess, IRX $\left(=\mathrm{L}_{I R} / \mathrm{L}_{F U V}\right)$. The threedimensional relation can be expressed in terms of empirical functions, where IRX is a function of NUV-3.6 $\mu \mathrm{m}$ (or more weakly with other colours) and $\mathrm{D}_{n}(4000)$. They suggest that this relation can be explained primarily through SFH and dust attenuation, with both acting to steepen the optical-UV slope (as measured by the NUV-3.6 $\mu \mathrm{m}$ color), but only attenuation increasing the IR flux and hence IRX (Johnson et al.2006).
A similar analysis was presented at the workshop by D. Dale using the LVL survey (see Section 3.3.1, which consists of a statistically complete set of star-forming galaxies, nearly two-thirds of which are dwarf/irregular systems. Figure 26 shows the ratio of far-ultraviolet-to-near-infrared luminosity as a function of the (perpendicular) distance from the starburst curve (e.g. Calzetti 1997; Meurer et al. 1999) for the LVL galaxies, with the far-ultraviolet emission is corrected for attenuation using the infrared-to-ultravioletbased recipe formulated in Buat et al. (2005). By correcting for dust, the FUV/3.6 $\mu \mathrm{m}$ ratio measures only the ratio of past-to-present star formation, sometimes referred to as the birthrate parameter (see also, for example, Boselli et al. 2001, Cortese et al. 2006). This ratio represents the birthrate parameter since the far-ultraviolet traces star formation over 100 Myr timescales whereas the near-infrared probes the total stellar mass built up over much longer timescales. This plot is as such a compression of the plane discussed by Johnson et al. (2007a), and shows a clear trend, with lower birthrate systems exhibiting larger distances from the starburst trend, consistent with the study of Kong et al. (2004). To further quantify this, theoretical models with solar metallicity, $1 M_{\odot} \mathrm{yr}^{-1}$ continuous star formation curves assuming a double power law initial mass function, with $\alpha_{1, \mathrm{IMF}}=1.3$ for $0.1<m / M_{\odot}<0.5$ and $\alpha_{2, \mathrm{IMF}}=2.3$ for $0.5<m / M_{\odot}<100$ were run (Vázquez and Leitherer 2005) and were matched with their determined FUV/3.6 $\mu \mathrm{m}$ ratio on the right axis, demonstrating that those with the oldest SFH (i.e. lowest birthrate parameters) lie furthest from the theoretical starburst curve.

\subsubsection{Star Formation Rate from the IR}

One of the most commonly extracted galaxy properties from the IR is the star formation rate. As discussed above, recently formed stellar populations tend to be more obscured than older stellar populations. They are also more luminous and emit more in the ultraviolet where dust opacity peaks, and thus dust emission is in principle a good tracer of recent star formation, assuming a simple calorimetric situation. It is these assumptions that lead to the widely-used Kennicutt (1998) relation between total IR luminosity $(8-1000 \mu \mathrm{m})$ and star formation rate.

Of course the situation is more complex than this, as discussed in the same work. A range of ages contribute to dust heating (Kennicutt et al. 2009), and star forming regions in galaxies suffer a range of obscurations, from totally obscured (ULIRGs) to unobscured (blue compact dwarfs). It is for this reason that this relation has been re-examined and empirically calibrated with new data from Spitzer. In general, all recent studies have found that the IR can be used as a SFR indicator, albeit without a direct one-to-one connection. Complications arise due to the correlation of SFR, 
luminosity, and galaxy gas and dust masses, and possible non-linearities due to metallicity effects (Wu et al.2005).

More specifically, Calzetti et al. (2007) using spatially resolved observations, found strong correlations between the $24 \mu \mathrm{m}$ and $\mathrm{P} \alpha$ luminosity densities (a proxy for SFR, assuming little attenuation in the near-IR), and a correlation between the $8 \mu \mathrm{m}$ and $\mathrm{P} \alpha$ luminosity densities, though this failed at low metallicities. Using these, they were able to create new calibrations for SFR versus $24 \mu \mathrm{m}$ luminosity, and SFR versus $24 \mu \mathrm{m}$ and observed $\mathrm{H} \alpha$ luminosities, with the latter relation accounting better for the escaping radiation not accounted for by the dust emission. Rieke et al. (2009) took this further, showing that for higher IR luminosity objects, $\mathrm{P} \alpha$ was no longer a good tracer for SFR as even it was obscured, and gave their own calibrations for SFR with the IR luminosities.

On galactic scales, Zhu et al. (2008) showed with a larger galaxy sample from SWIRE that the Calzetti et al. (2007) relations between $24 \mu \mathrm{m}$ luminosity and extinction corrected $\mathrm{H} \alpha$ luminosity hold, and thus $\mathrm{L}(24 \mu \mathrm{m})$ is a good SFR indicator. In addition they also showed that $24 \mu \mathrm{m}$ is well correlated with $70 \mu \mathrm{m}$ and total IR luminosities, indicating that these too can be used as SFR indicators, albeit with larger scatter.

The $8 \mu \mathrm{m}$ (and other PAH bands) and longer wavelength observations, such as the Spitzer $160 \mu \mathrm{m}$ band, are observed to be correlated with each other (Bendo et al. 2008), and are thought to be more associated with the cooler diffuse ISM. While the diffuse ISM is heated by the radiation from star forming regions, it is also heated by the diffuse radiation field from older stars, meaning that these bands are not as strong SFR traces, especially at low SFRs.

\subsection{Fitting the full UV to FIR SED}

Fitting a self-consistent model over the divide between stellar and dust emission in the SED is of course one of the ultimate goals of SED fitting. However, while modelling efforts are well-underway (see Section 2.2.3), unfortunately, few authors have attempted to apply these to large samples of galaxies. Inversion techniques are not applicable here, as the problem is highly non-linear with many free parameters and therefore time consuming. Even for calculation of a library of model galaxies, it is challenging to provide models that are sufficiently simple, complete and fast to make this a practical possibility.

We here need to bypass instances where modellers test their codes on single galaxies (e.g. Silva et al. 1998 Popescu et al. 2000; Groves et al. 2008). This is of course a most valuable and necessary step to make sure that the model does bear on our understanding of reality and to further our knowledge of the underlying physics. Most of the results from these studies have been presented above in Section 2 For the potential novice reader of this manuscript we nevertheless emphasize at this point that proper filter convolution and $\chi^{2}$ fitting cannot be replaced by by-eye passing of spectra through photometric data points.

Using the model by da Cunha et al. (2008), in a follow-up paper da Cunha et al. (2010) have demonstrated the strength of fitting the full SED from UV to IR wavelengths. By fitting the full SEDs of $\sim 3000$ galaxies with GALEX, SDSS, 2MASS, and IRAS data, they were able to determine the star formation rate, the star formation history as measured by the specific star formation ( $\psi_{\mathrm{s}}=$ current $0-10^{8} \mathrm{Myr}$ star formation rate divided by the past average star formation rate), dust and stellar masses and other parameters. They found a strong correlation of dust mass $\left(M_{\mathrm{d}}\right)$ to star formation rate $\left(\psi\right.$, in $\left.\mathrm{M}_{\odot} \mathrm{yr}^{-1}\right)$, shown below in equation 6), as well as relations between the dust to stellar mass ratio and $\psi_{\mathrm{s}}$, and the fraction of IR emission arising from the diffuse ISM and $\psi_{\mathrm{s}}$.

$M_{\mathrm{d}}=(1.28 \pm 0.02) \times 10^{7} \psi^{1.11 \pm 0.01} \mathrm{M}_{\odot}$

This work demonstrates clearly the connection between dust mass, star formation history and stellar evolution.

Iglesias-Páramo et al. (2007) have gone to the length of using the GRASIL code (Silva et al. 1998) to compute a library of 5000 model galaxies and then use Bayesian inference to derive the properties of their sample. Their general results agreed well with independent studies by other authors, thus lending support to the notion that full SED fitting is a reliable tool to derive galaxy properties. More importantly in the present context, they show that their reduced $\chi^{2}$ distribution has a median value of 2.6, albeit with a long tail extending well above 10 . Thus the GRASIL library is found to reproduce their sample fairly well. Nevertheless, from the point of view of reliable SED fitting tools, a more thorough analysis of the outliers (model uncertainties, incomplete libraries, AGN, etc.) would be valuable, not only in this but in many other works.

Noll et al. (2009) present a new code, which they call "CIGALE", which effectively computes a library of model galaxies and then uses a modified version of the Bayesian inference described in Section 4.5 to determine the galaxy properties. Diagnostic plots like their Figure 14, which shows the residuals between best fit model and data for their full sample, are a very useful tool to understand model systematics. In their case for example, they conclude that "For MIPS $160 \mu \mathrm{m}$ the significant deviations can partly be explained by the lacking flexibility of the one-parameter models of Dale and Helou (2002)."

As shown by the last two examples, most UV-FIR SED fitting codes are still in their testing phase and have mostly been used to confirm results already obtained from more traditional single-tracer analyses. The large number of derived parameters and our still limited knowledge of their respective degeneracies and systematic uncertainties make it difficult to go a step further and fully use the full power of 
SED fitting. Indeed, for the moment it is still questionable whether it is not more fruitful to use a combination of single tracers to derive one property well (e.g. Kennicutt et al. 2009, for SFR). On the long run, however, SED fitting holds the promise to provide a large set of galaxy properties for large samples. Self-consistent inter-comparison of sub-samples with different properties, such as masses and SFRs, and the exploitation of constraints on hitherto unconstrainable parameters, such as the relative weights of young, intermediate age and old populations, are an exciting avenue to explore further in the future.

\section{Conclusions}

We have presented an overview of some of the achievements and challenges related to fitting the Spectral Energy Distributions of galaxies. SED fitting can be used effectively to derive a range of physical properties of galaxies, such as redshift, stellar masses, star formation rates, dust masses, and metallicities, with care taken not to over-interpret the available data. To allow for more progress in galaxy evolution studies from SED fitting, we suggest two main areas. On the one hand there still exist many specific issues such as estimating the age of the oldest stars in a galaxy, finer details of dust properties and dust-star geometry, and the influences of poorly understood, luminous stellar types and phases. The challenge for the coming years will be to improve both the models and the observational data sets to resolve these uncertainties. On the other hand, the robustness and accuracy of SED-fitting-derived properties still need to be assessed more completely. The challenge here is to develop and understand the interplay between the fitting routines and the available data and models.

In the hope of accompanying these challenges, the present review will be made available on a webpage (sedfitting.org) together with links to relevant models, fitting codes and datasets. We would like to encourage the community to send in suggestions for additions and changes to the tex through this webpage. The intention is twofold: 1) We hope to bolster the information currently available in this review and keep it up to date over the coming years. 2) Due to our bias to the workshop participants we did mention many important works in passing, or indeed missed them. We therefore hope that particularly those members of the community whom we missed will take to opportunity to add their part of the story, thus expanding the current text beyond its original scope.

\section{Acknowledgements}

${ }^{6}$ Please note that reference to the online text should always include a citation to the present work.
The authors of this review and organizers of the workshop would like to thank the Lorentz center for making this workshop possible and for providing a first-class meeting environment. We also thank NOVA for additional support.

We would like to thank the participants in the workshop for their motivation and for sharing their expertise, without which this review could not have been written. The talks and discussions of the attendees formed a large part of this review. Furthermore, we are deeply indebted to the following participants for supplying many pages worth of text: $\mathrm{H}$. Aussel, N. Ball, M. Brodwin, S. Charlot, L. Dunne, I. Ferreras, V. Margoniner, M. Polletta, A. Sajina, J.D. Smith, P. Oesch, V. Wild, C. Wolf, S. Zibetti. We thank N. Ball, J. Brinchmann, E. da Cunha, S. Charlot, M. Dopita, L. Dunne, I. Ferreras, P. Oesch, and V. Wild for custom making their figures.

We thank an anonymous referee for a critical and thorough reading of the manuscript, which led us to clarify several important points in the text. We also thank L. Spezzi for a careful reading of an early version of the manuscript.

This research has made use of NASA's Astrophysics Data System Bibliographic Services. 


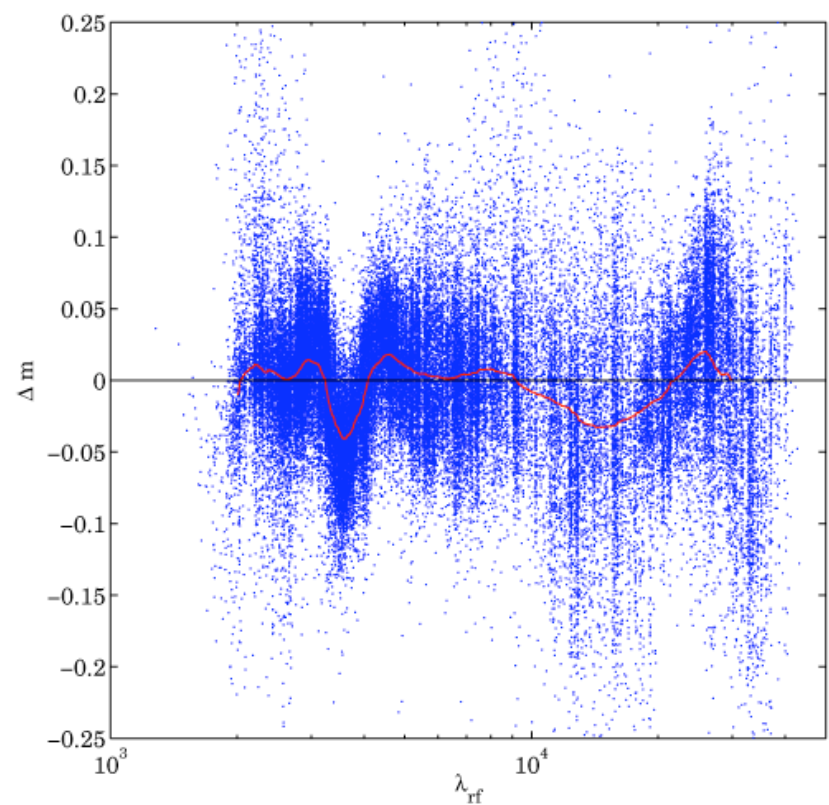

Fig. 17 Rest-frame residuals $(\Delta \mathrm{m}=$ (template galaxy)/template) for all galaxies with reliable redshifts from the zCOSMOS sample. The feature with the strongest significance is at around $3500 \AA$, where the templates are too faint by $0.05 \mathrm{mag}$ compared to the data. Additional nebular emission was added to the original SPS templates for this plot. [Courtesy P. Oesch]

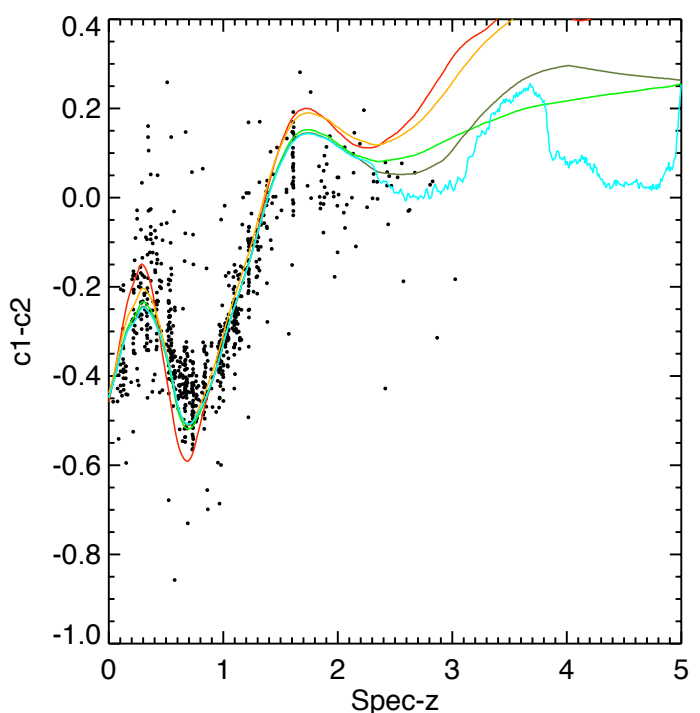

Fig. 18 IRAC [3.6]-[4.5] color-redshift plot for a sample of GOODS galaxies. The solid curves show the change in colours with redshift of five different empirical template SEDs. [Courtesy M. Brodwin] 

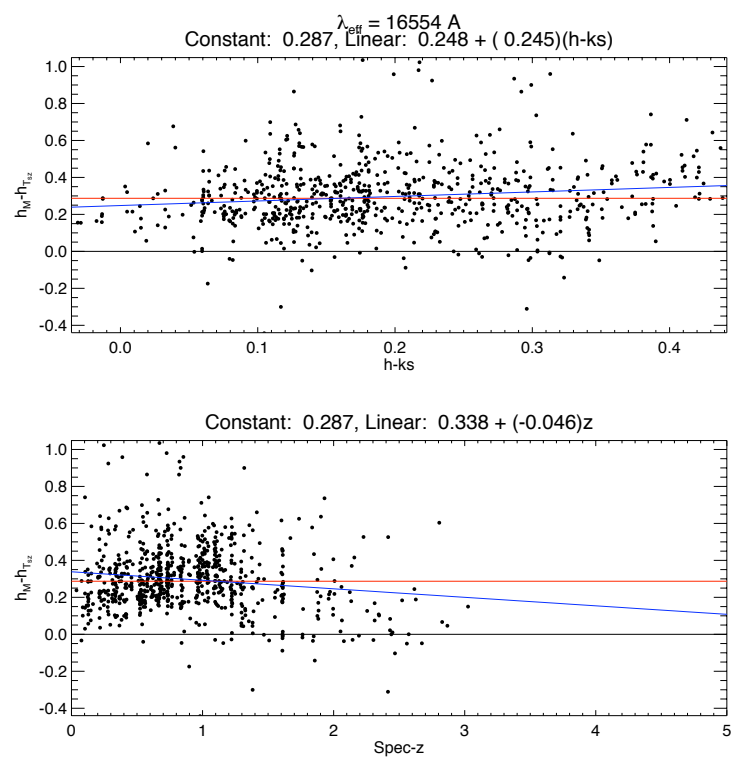

Fig. $19 H$-band residuals vs. color and redshift in a sample of GOODS galaxies. An effective offset of $\approx 0.3$ mags is apparent. [Courtesy M. Brodwin]

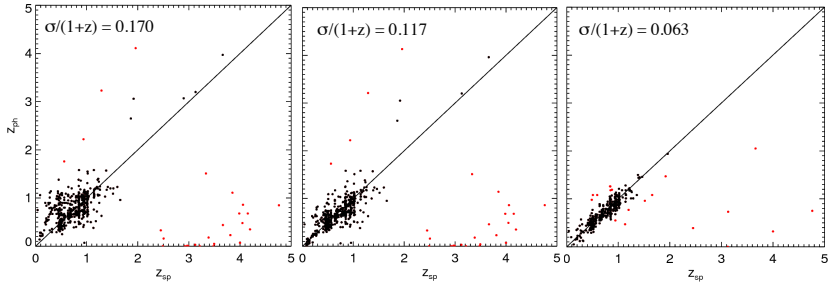

Fig. 20 Iterative improvement in photometric redshift estimation via this simple calibration technique. [Courtesy M. Brodwin]
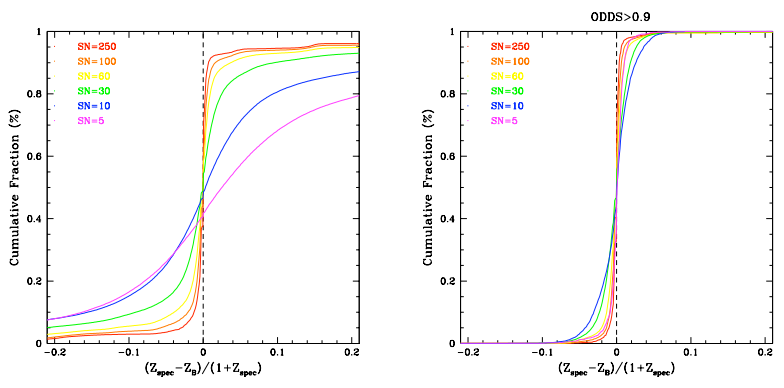

Fig. 21 Cumulative fraction of objects with $\delta_{z}$ smaller than agiven value. Red line indicates the simulation in which all galaxies have been set to have $\mathrm{SN}=250$ in all $\mathrm{BVRz}$; orange indicates a simulation ith $\mathrm{SN}=100$; and so on. Right panel shows all galaxies, and left panel shows galaxies with $O D D S>0.9$. Note that only $6.4 \%$ and $1.2 \%$ respectively of objects with $\mathrm{SN}=10,5$ have $O D D S>0.9$ (figure from Margoniner and Wittman 2008) [Courtesy V. Margoniner].
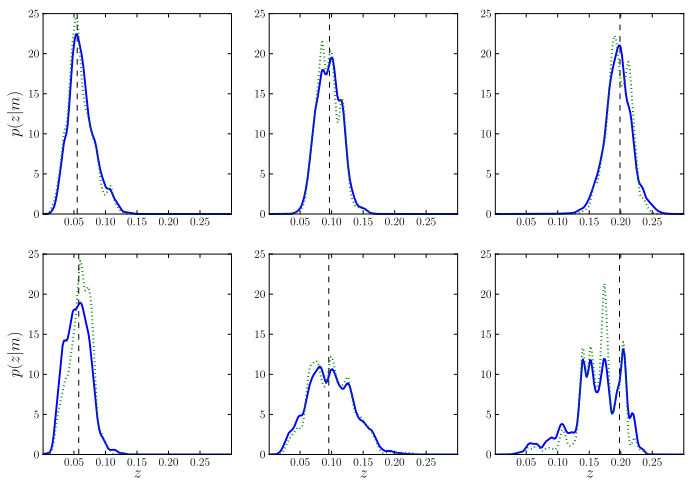

Fig. 22 The probability density as a function of the redshift for early- and late-type galaxies (upper and lower panels, respectively) at different distances marked by the vertical dashed lines. For every object, the dotted line shows the empirical relation of $p\left(z \mid \boldsymbol{x}=\boldsymbol{m}_{q}\right)$, and the solid line illustrates the final result of $p\left(z \mid \boldsymbol{y}=\boldsymbol{m}_{q}, M\right)$ after properly folding in the photometric uncertainties via the mapping in the model (Budavári 2009a, figure from) [Courtesy T. Budavari]. 


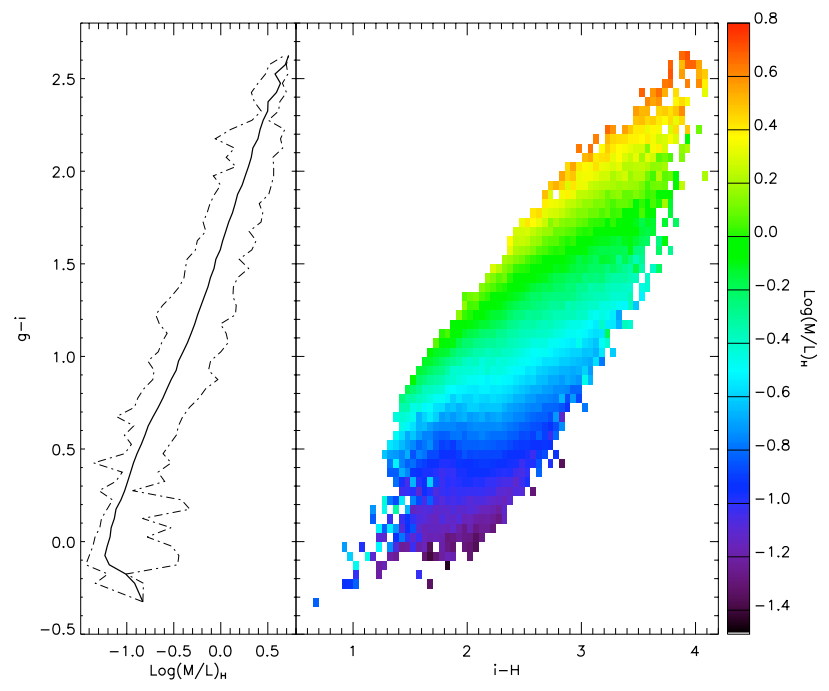

Fig. 23 Monte Carlo library of 50,000 models created from the Version 2007 of Bruzual and Charlot (2003). The $(g-i, i-H)$ space is binned in cells of $0.05 \mathrm{mag} \times 0.05 \mathrm{mag}$ and marginalized over $M / L$ in each cell. The right panel shows $M / L_{H}$ as a function of $(g-i, i-H)$. The scatter at each position is typically $0.11 \mathrm{dex}$, with peaks at $\sim 0.2$ dex in the bluest corner. The left panel shows $M / L$ as a function of $g-i$ (median value marginalized in a given $g-i$ bin). The minimum and maximum $M / L$ values derived from the right panel at given $g-i$ are displayed by dashed lines. Figure from Zibetti et al. (2009) [Courtesy S. Zibetti].
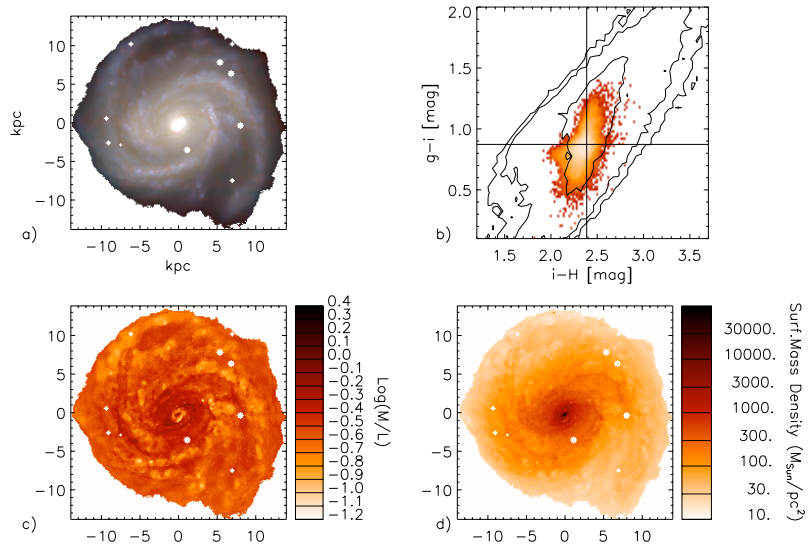

Fig. 24 Panel a) shows the color-composite RGB image ( $H$ in red channel, $i$ in green and $g$ in blue) after adaptive smoothing. Panel b) plots the distribution of pixels in color-color space (log intensity scale). The cross marks the position of the global colors of the galaxy. The over-plotted contours show the number density distribution of models from the library described above. Interestingly, observed colors are reproduced by the models over the full range. Panel c) shows the mass-to-light ratio map derived with the method described above. Finally, Panel d) displays the stellar mass surface density map, obtained by multiplying the $H$ band surface brightness times $M / L$. Images a) and d) show that the galactic structure is much smoother in the mass image, where spiral arms have much less contrast with respect to what the light shows. Figure from $\mathrm{Zi-}$ betti et al. (2009) [Courtesy S. Zibetti].

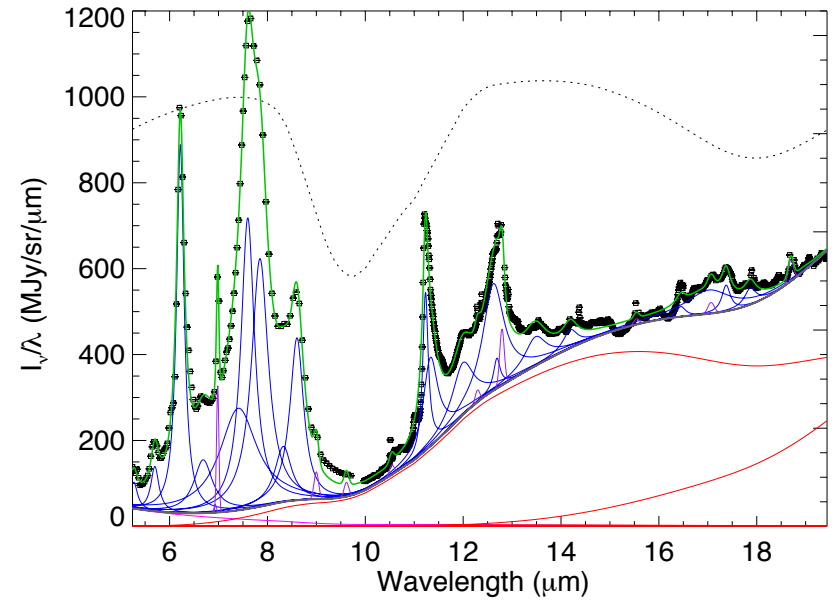

Fig. 25 An example of combining Spitzer-IRS data with a PAHFIT mid-infrared model. The IRS data arise from the central region of M82 and are marked by the boxes. The PAHFIT final spectrum passes through these points (green line) and can be seperated into PAH features (broad blue curves), fine-structure emission lines (purple narrow cuves), hot dust continuum (underlying red cuves) all experiencing broad dust extinction, especially the pronounced silicate 10 and $18 \mu \mathrm{m}$ features (represented by the dotted curve). Data are from (Beirão et al.|2008). 


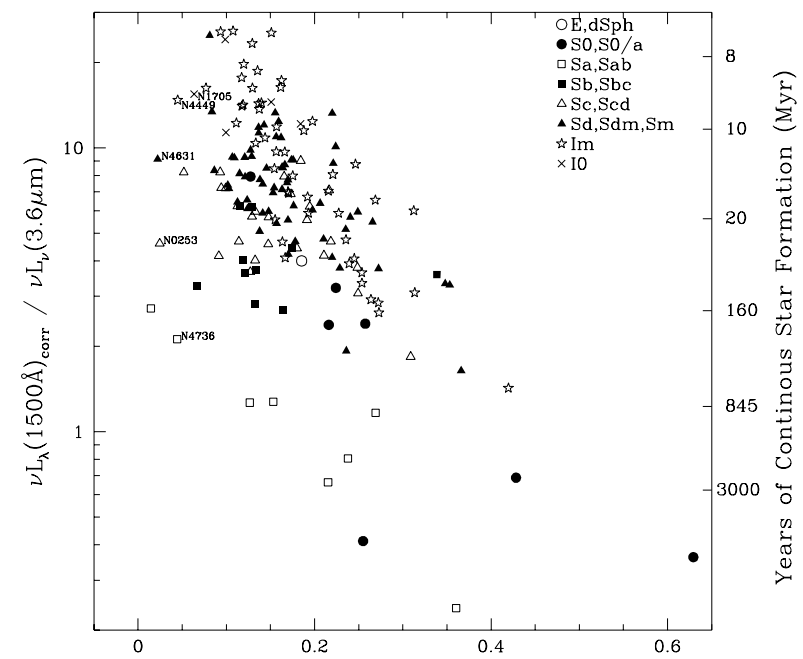

Perpendicular Distance from Starburst Curve (dex)

Fig. 26 The dependence of galaxy star formation history as a function of distance from the starburst relation of Calzetti (1997);

Meurer et al. (1999). The lefthand axis is an observable diagnostic of the birthrate parameter, the current star formation rate normalized to the average star formation rate. The righthand axis shows the number of years (continuous) star formation has been occurring, as measured from theoretical spectra. 


\section{References}

Abazajian, K.N., Adelman-McCarthy, J.K., Agüeros, M.A., Allam, S.S., Allende Prieto, C., An, D., Anderson, K.S.J., Anderson, S.F., Annis, J., Bahcall, N.A., Bailer-Jones, C.A.L., Barentine, J.C., Bassett, B.A., Becker, A.C., Beers, T.C., Bell, E.F., Belokurov, V., Berlind, A.A., Berman, E.F., Bernardi, M., Bickerton, S.J., Bizyaev, D., Blakeslee, J.P., Blanton, M.R., Bochanski, J.J., Boroski, W.N., Brewington, H.J., Brinchmann, J., Brinkmann, J., Brunner, R.J., Budavári, T., Carey, L.N., Carliles, S., Carr, M.A., Castander, F.J., Cinabro, D., Connolly, A.J., Csabai, I., Cunha, C.E., Czarapata, P.C., Davenport, J.R.A., de Haas, E., Dilday, B., Doi, M., Eisenstein, D.J., Evans, M.L., Evans, N.W., Fan, X., Friedman, S.D., Frieman, J.A., Fukugita, M., Gänsicke, B.T., Gates, E., Gillespie, B., Gilmore, G., Gonzalez, B., Gonzalez, C.F., Grebel, E.K., Gunn, J.E., Györy, Z., Hall, P.B., Harding, P., Harris, F.H., Harvanek, M., Hawley, S.L., Hayes, J.J.E., Heckman, T.M., Hendry, J.S., Hennessy, G.S., Hindsley, R.B., Hoblitt, J., Hogan, C.J., Hogg, D.W., Holtzman, J.A., Hyde, J.B., Ichikawa, S., Ichikawa, T., Im, M., Ivezić, Ž., Jester, S., Jiang, L., Johnson, J.A., Jorgensen, A.M., Jurić, M., Kent, S.M., Kessler, R., Kleinman, S.J., Knapp, G.R., Konishi, K., Kron, R.G., Krzesinski, J., Kuropatkin, N., Lampeitl, H., Lebedeva, S., Lee, M.G., Lee, Y.S., Leger, R.F., Lépine, S., Li, N., Lima, M., Lin, H., Long, D.C., Loomis, C.P., Loveday, J., Lupton, R.H., Magnier, E., Malanushenko, O., Malanushenko, V., Mandelbaum, R., Margon, B., Marriner, J.P., MartínezDelgado, D., Matsubara, T., McGehee, P.M., McKay, T.A., Meiksin, A., Morrison, H.L., Mullally, F., Munn, J.A., Murphy, T., Nash, T., Nebot, A., Neilsen, E.H., Newberg, H.J., Newman, P.R., Nichol, R.C., Nicinski, T., NietoSantisteban, M., Nitta, A., Okamura, S., Oravetz, D.J., Ostriker, J.P., Owen, R., Padmanabhan, N., Pan, K., Park, C., Pauls, G., Peoples, J., Percival, W.J., Pier, J.R., Pope, A.C., Pourbaix, D., Price, P.A., Purger, N., Quinn, T., Raddick, M.J., Fiorentin, P.R., Richards, G.T., Richmond, M.W., Riess, A.G., Rix, H., Rockosi, C.M., Sako, M., Schlegel, D.J., Schneider, D.P., Scholz, R., Schreiber, M.R., Schwope, A.D., Seljak, U., Sesar, B., Sheldon, E., Shimasaku, K., Sibley, V.C., Simmons, A.E., Sivarani, T., Smith, J.A., Smith, M.C., Smolčić, V., Snedden, S.A., Stebbins, A., Steinmetz, M., Stoughton, C., Strauss, M.A., Subba Rao, M., Suto, Y., Szalay, A.S., Szapudi, I., Szkody, P., Tanaka, M., Tegmark, M., Teodoro, L.F.A., Thakar, A.R., Tremonti, C.A., Tucker, D.L., Uomoto, A., Vanden Berk, D.E., Vandenberg, J., Vidrih, S., Vogeley, M.S., Voges, W., Vogt, N.P., Wadadekar, Y., Watters, S., Weinberg, D.H., West, A.A., White, S.D.M., Wilhite, B.C., Wonders, A.C., Yanny, B., Yocum, D.R., York, D.G., Zehavi, I., Zibetti, S., Zucker, D.B.: Astrophys. J. Suppl. Ser. 182, 543 (2009)

Adelman-McCarthy, J.K., et al.: Astrophys. J. Suppl. Ser. 172, 634 (2007)

Agladze, N.I., Sievers, A.J., Jones, S.A., Burlitch, J.M., Beckwith, S.V.W.: Astrophys. J. 462, 1026 (1996)

Anders, P., Lamers, H.J.G.L.M., Baumgardt, H.: Astron. Astrophys. 502, 817 (2009)

Andriesse, C.D.: Astron. Astrophys. 37, 257 (1974)

Arnouts, S., Cristiani, S., Moscardini, L., Matarrese, S., Lucchin, F., Fontana, A., Giallongo, E.: Mon. Not. R. Astron. Soc. 310, 540 (1999)
Arnouts, S., Walcher, C.J., Le Fèvre, O., Zamorani, G., Ilbert, O., Le Brun, V., Pozzetti, L., Bardelli, S., Tresse, L., Zucca, E., Charlot, S., Lamareille, F., McCracken, H.J., Bolzonella, M., Iovino, A., Lonsdale, C., Polletta, M., Surace, J., Bottini, D., Garilli, B., Maccagni, D., Picat, J.P., Scaramella, R., Scodeggio, M., Vettolani, G., Zanichelli, A., Adami, C., Cappi, A., Ciliegi, P., Contini, T., de la Torre, S., Foucaud, S., Franzetti, P., Gavignaud, I., Guzzo, L., Marano, B., Marinoni, C., Mazure, A., Meneux, B., Merighi, R., Paltani, S., Pellò, R., Pollo, A., Radovich, M., Temporin, S., Vergani, D.: Astron. Astrophys. 476, 137 (2007)

Baes, M., Davies, J.I., Dejonghe, H., Sabatini, S., Roberts, S., Evans, R., Linder, S.M., Smith, R.M., de Blok, W.J.G.: Mon. Not. R. Astron. Soc. 343, 1081 (2003)

Baes, M., Dejonghe, H.: Mon. Not. R. Astron. Soc. 326, 722 (2001a)

Baes, M., Dejonghe, H.: Mon. Not. R. Astron. Soc. 326, 733 (2001b)

Baes, M., Stamatellos, D., Davies, J.I., Whitworth, A.P., Sabatini, S., Roberts, S., Linder, S.M., Evans, R.: New Astronomy 10, 523 (2005)

Baldwin, J.A., Phillips, M.M., Terlevich, R.: Publ. Astron. Soc. Pac. 93, 5 (1981)

Ball, N.M., Brunner, R.J., Myers, A.D., Strand, N.E., Alberts, S.L., Tcheng, D.: Astrophys. J. 683, 12 (2008a)

Ball, N.M., Loveday, J., Brunner, R.J.: Mon. Not. R. Astron. Soc. 383, 907 (2008b)

Ball, N.M., Loveday, J., Fukugita, M., Nakamura, O., Okamura, S., Brinkmann, J., Brunner, R.J.: Mon. Not. R. Astron. Soc. 348, 1038 (2004)

Balogh, M.L., Morris, S.L., Yee, H.K.C., Carlberg, R.G., Ellingson, E.: Astrophys. J. 527, 54 (1999)

Barbaro, C., Bertelli, C.: Astron. Astrophys. 54, 243 (1977)

Baum, W.A.: Astron. J. 62, 6 (1957)

Bauschlicher, C.W., Peeters, E., Allamandola, L.J.: Astrophys. J. 697, 311 (2009)

Beirão, P., Brandl, B.R., Appleton, P.N., Groves, B., Armus, L., Förster Schreiber, N.M., Smith, J.D., Charmandaris, V., Houck, J.R.: Astrophys. J. 676, 304 (2008)

Bell, E.F., Baugh, C.M., Cole, S., Frenk, C.S., Lacey, C.G.: Mon. Not. R. Astron. Soc. 343, 367 (2003b)

Bell, E.F., de Jong, R.S.: Astrophys. J. 550, 212 (2001)

Bell, E.F., McIntosh, D.H., Katz, N., Weinberg, M.D.: Astrophys. J. Suppl. Ser. 149, 289 (2003a)

Bell, E.F., Zheng, X.Z., Papovich, C., Borch, A., Wolf, C., Meisenheimer, K.: Astrophys. J. 663, 834 (2007)

Bendo, G.J., Draine, B.T., Engelbracht, C.W., Helou, G., Thornley, M.D., Bot, C., Buckalew, B.A., Calzetti, D., Dale, D.A., Hollenbach, D.J., Li, A., Moustakas, J.: Mon. Not. R. Astron. Soc. 389, 629 (2008)

Benítez, N.: Astrophys. J. 536, 571 (2000)

Berta, S.: Multiwavelength analyses of faint infrared galaxies. PhD thesis, Dipartimento di Astronomia, Univ. di Padova, Vicolo dell'Osservatorio 2, I-35122, Padova, Italy (2005)

Berta, S., Fritz, J., Franceschini, A., Bressan, A., Pernechele, C.: Astron. Astrophys. 403, 119 (2003)

Bianchi, S.: Astron. Astrophys. 490, 461 (2008)

Bianchi, S., Davies, J.I., Alton, P.B.: Astron. Astrophys. 359, 65 (2000)

Bianchi, S., Ferrara, A., Giovanardi, C.: Astrophys. J. 465, 127 (1996) 
Bjorkman, J.E., Wood, K.: Astrophys. J. 554, 615 (2001)

Blanton, M.R., Brinkmann, J., Csabai, I., Doi, M., Eisenstein, D., Fukugita, M., Gunn, J.E., Hogg, D.W., Schlegel, D.J.: Astron. J. 125, 2348 (2003)

Blanton, M.R., Roweis, S.: Astron. J. 133, 734 (2007)

Boissier, S., Gil de Paz, A., Boselli, A., Madore, B.F., Buat, V., Cortese, L., Burgarella, D., Muñoz-Mateos, J.C., Barlow, T.A., Forster, K., Friedman, P.G., Martin, D.C., Morrissey, P., Neff, S.G., Schiminovich, D., Seibert, M., Small, T., Wyder, T.K., Bianchi, L., Donas, J., Heckman, T.M., Lee, Y.W., Milliard, B., Rich, R.M., Szalay, A.S., Welsh, B.Y., Yi, S.K.: Astrophys. J. Suppl. Ser. 173, 524 (2007)

Bolzonella, M., Miralles, J.M., Pelló, R.: Astron. Astrophys. 363, 476 (2000)

Boquien, M., Duc, P., Braine, J., Brinks, E., Lisenfeld, U., Charmandaris, V.: Astron. Astrophys. 467, 93 (2007)

Boselli, A., Gavazzi, G., Donas, J., Scodeggio, M.: Astron. J. 121, 753 (2001)

Bressan, A., Granato, G.L., Silva, L.: Astron. Astrophys. 332, 135 (1998)

Brinchmann, J., Charlot, S., White, S.D.M., Tremonti, C., Kauffmann, G., Heckman, T., Brinkmann, J.: Mon. Not. R. Astron. Soc. 351, 1151 (2004)

Brinchmann, J., Kunth, D., Durret, F.: Astron. Astrophys. 485, 657 (2008)

Brodwin, M., Brown, M.J.I., Ashby, M.L.N., Bian, C., Brand, K., Dey, A., Eisenhardt, P.R., Eisenstein, D.J., Gonzalez, A.H., Huang, J.S., Jannuzi, B.T., Kochanek, C.S., McKenzie, E., Murray, S.S., Pahre, M.A., Smith, H.A., Soifer, B.T., Stanford, S.A., Stern, D., Elston, R.J.: Astrophys. J. 651, 791 (2006b)

Brodwin, M., Lilly, S.J., Porciani, C., McCracken, H.J., Le Fèvre, O., Foucaud, S., Crampton, D., Mellier, Y.: Astrophys. J. Suppl. Ser. 162, 20 (2006a)

Brunner, R.J., Connolly, A.J., Szalay, A.S., Bershady, M.A.: Astrophys. J. Lett. 482, L21 (1997)

Bruzual, G., Charlot, S.: Mon. Not. R. Astron. Soc. 344, 1000 (2003)

Bruzual A., G.: Astrophysics and Space Science Supplement 277, $221(2001)$

Bruzual A., G., Magris, G., Calvet, N.: Astrophys. J. 333, 673 (1988)

Buat, V., Iglesias-Páramo, J., Seibert, M., Burgarella, D., Charlot, S., Martin, D.C., Xu, C.K., Heckman, T.M., Boissier, S., Boselli, A., Barlow, T., Bianchi, L., Byun, Y.I., Donas, J., Forster, K., Friedman, P.G., Jelinski, P., Lee, Y.W., Madore, B.F., Malina, R., Milliard, B., Morissey, P., Neff, S., Rich, M., Schiminovitch, D., Siegmund, O., Small, T., Szalay, A.S., Welsh, B., Wyder, T.K.: Astrophys. J. Lett. 619, L51 (2005)

Budavári, T.: Astrophys. J. 695, 747 (2009a)

Budavári, T., Szalay, A.S., Connolly, A.J., Csabai, I., Dickinson, M.: Astron. J. 120, 1588 (2000)

Budavári, T., Wild, V., Szalay, A.S., Dobos, L., Yip, C.W.: Mon. Not. R. Astron. Soc. 394, 1496 (2009b)

Bundy, K., Ellis, R.S., Conselice, C.J., Taylor, J.E., Cooper, M.C., Willmer, C.N.A., Weiner, B.J., Coil, A.L., Noeske, K.G., Eisenhardt, P.R.M.: Astrophys. J. 651, 120 (2006)

Burgarella, D., Buat, V., Iglesias-Páramo, J.: Mon. Not. R. Astron. Soc. 360, 1413 (2005)

Burstein, D., Faber, S.M., Gaskell, C.M., Krumm, N.: Astrophys. J. 287, 586 (1984)

Buzzoni, A.: Astrophys. J. Suppl. Ser. 71, 817 (1989)
Calura, F., Pipino, A., Matteucci, F.: Astron. Astrophys. 479, 669 (2008)

Calzetti, D.: Astron. J. 113, 162 (1997)

Calzetti, D., Armus, L., Bohlin, R.C., Kinney, A.L., Koornneef, J., Storchi-Bergmann, T.: Astrophys. J. 533, 682 (2000)

Calzetti, D., Kennicutt, R.C., Engelbracht, C.W., Leitherer, C., Draine, B.T., Kewley, L., Moustakas, J., Sosey, M., Dale, D.A., Gordon, K.D., Helou, G.X., Hollenbach, D.J., Armus, L., Bendo, G., Bot, C., Buckalew, B., Jarrett, T., Li, A., Meyer, M., Murphy, E.J., Prescott, M., Regan, M.W., Rieke, G.H., Roussel, H., Sheth, K., Smith, J.D.T., Thornley, M.D., Walter, F.: Astrophys. J. 666, 870 (2007)

Calzetti, D., Kinney, A.L., Storchi-Bergmann, T.: Astrophys. J. 429, 582 (1994)

Cappellari, M., Bacon, R., Bureau, M., Damen, M.C., Davies, R.L., de Zeeuw, P.T., Emsellem, E., Falcón-Barroso, J., Krajnović, D., Kuntschner, H., McDermid, R.M., Peletier, R.F., Sarzi, M., van den Bosch, R.C.E., van de Ven, G.: Mon. Not. R. Astron. Soc. 366, 1126 (2006)

Cardelli, J.A., Clayton, G.C., Mathis, J.S.: Astrophys. J. 345, 245 (1989)

Carter, D.: Mon. Not. R. Astron. Soc. 178, 137 (1977)

Catelan, M.: Astrophys. Space Sci., 18 (2009)

Cenarro, A.J., Gorgas, J., Cardiel, N., Vazdekis, A., Peletier, R.F.: Mon. Not. R. Astron. Soc. 329, 863 (2002)

Cenarro, A.J., Peletier, R.F., Sánchez-Blázquez, P., Selam, S.O., Toloba, E., Cardiel, N., Falcón-Barroso, J., Gorgas, J., JiménezVicente, J., Vazdekis, A.: Mon. Not. R. Astron. Soc. 374, 664 (2007)

Cerviño, M., Luridiana, V.: Astron. Astrophys. 413, 145 (2004)

Cerviño, M., Luridiana, V.: Astron. Astrophys. 451, 475 (2006)

Cerviño, M., Mas-Hesse, J.M., Kunth, D.: Astron. Astrophys. 392, 19 (2002)

Cesetti, M., Ivanov, V.D., Morelli, L., Pizzella, A., Buson, L., Corsini, E.M., Dalla Bontà, E., Stiavelli, M., Bertola, F.: Astron. Astrophys. 497, 41 (2009)

Chabrier, G.: Astrophys. J. 554, 1274 (2001)

Chabrier, G.: Publ. Astron. Soc. Pac. 115, 763 (2003)

Chakrabarti, S., Whitney, B.A.: Astrophys. J. 690, 1432 (2009)

Charlot, S., Bruzual, A.G.: Astrophys. J. 367, 126 (1991)

Charlot, S., Fall, S.M.: Astrophys. J. 539, 718 (2000)

Charlot, S., Longhetti, M.: MNRAS 323, 887 (2001). (c) 2001 The Royal Astronomical Society

Chary, R., Elbaz, D.: Astrophys. J. 556, 562 (2001)

Chavez, M., Bertone, E., Morales-Hernandez, J., Bressan, A.: Astrophys. J. 700, 694 (2009)

Chilingarian, I., Prugniel, P., Sil'Chenko, O., Koleva, M.: In: Vazdekis, A., Peletier, R.F. (eds.) IAU Symposium. IAU Symposium, vol. 241, 175 (2007)

Chiosi, C., Bertelli, G., Bressan, A.: Astron. Astrophys. 196, 84 (1988)

Cid Fernandes, R., Mateus, A., Sodré, L., Stasińska, G., Gomes, J.M.: Mon. Not. R. Astron. Soc. 358, 363 (2005)

Cimatti, A.: In: G. Giobbi, A. Tornambe, G. Raimondo, M. Limongi, L. A. Antonelli, N. Menci, \& E. Brocato (ed.) American Institute of Physics Conference Series. American Institute of Physics Conference Series, vol. 1111, 191 (2009)

Cirasuolo, M., McLure, R.J., Dunlop, J.S., Almaini, O., Foucaud, S., Simpson, C.: Mon. Not. R. Astron. Soc. 401, 1166 (2010) 
Cirasuolo, M., McLure, R.J., Dunlop, J.S., Almaini, O., Foucaud, S., Smail, I., Sekiguchi, K., Simpson, C., Eales, S., Dye, S., Watson, M.G., Page, M.J., Hirst, P.: Mon. Not. R. Astron. Soc. 380, 585 (2007)

Clements, D.L., Dunne, L., Eales, S.A.: ArXiv e-prints (2009, in press)

Coelho, P.: In: Giobbi, G., Tornambe, A., Raimondo, G., Limongi, M., Antonelli, L.A., Menci, N., Brocato, E. (eds.) American Institute of Physics Conference Series. American Institute of Physics Conference Series, vol. 1111, 67 (2009)

Coelho, P., Barbuy, B., Meléndez, J., Schiavon, R.P., Castilho, B.V.: Astron. Astrophys. 443, 735 (2005)

Coelho, P., Bruzual, G., Charlot, S., Weiss, A., Barbuy, B., Ferguson, J.W.: Mon. Not. R. Astron. Soc. 382, 498 (2007)

Cole, S., Lacey, C.G., Baugh, C.M., Frenk, C.S.: Mon. Not. R. Astron. Soc. 319, 168 (2000)

Coleman, G.D., Wu, C.C., Weedman, D.W.: Astrophys. J. Suppl. Ser. 43, 393 (1980)

Collister, A.A., Lahav, O.: Publ. Astron. Soc. Pac. 116, 345 (2004)

Connolly, A.J., Budavári, T., Szalay, A.S., Csabai, I., Brunner, R.J.: In: Weymann, R., Storrie-Lombardi, L., Sawicki, M., Brunner, R. (eds.) Photometric Redshifts and the Detection of High Redshift Galaxies. Astronomical Society of the Pacific Conference Series, vol. 191, 13 (1999)

Connolly, A.J., Csabai, I., Szalay, A.S., Koo, D.C., Kron, R.G., Munn, J.A.: Astron. J. 110, 2655 (1995a)

Connolly, A.J., Szalay, A.S., Bershady, M.A., Kinney, A.L., Calzetti, D.: Astron. J. 110, 1071 (1995b)

Conroy, C., Gunn, J.E.: Astrophys. J. 712, 833 (2010b)

Conroy, C., Gunn, J.E., White, M.: Astrophys. J. 699, 486 (2009)

Conroy, C., White, M., Gunn, J.E.: Astrophys. J. 708, 58 (2010a)

Cortese, L., Boselli, A., Buat, V., Gavazzi, G., Boissier, S., Gil de Paz, A., Seibert, M., Madore, B.F., Martin, D.C.: Astrophys. J. 637, 242 (2006)

Cox, T.J., Jonsson, P., Primack, J.R., Somerville, R.S.: Mon. Not. R. Astron. Soc. 373, 1013 (2006)

Czerny, B., Li, J., Loska, Z., Szczerba, R.: Mon. Not. R. Astron. Soc. 348, L54 (2004)

da Cunha, E., Charlot, S., Elbaz, D.: Mon. Not. R. Astron. Soc. 388, 1595 (2008)

da Cunha, E., Eminian, C., Charlot, S., Blaizot, J.: ArXiv e-prints (2010, in press)

Dalcanton, J.J., Williams, B.F., Seth, A.C., Dolphin, A., Holtzman, J., Rosema, K., Skillman, E.D., Cole, A., Girardi, L., Gogarten, S.M., Karachentsev, I.D., Olsen, K., Weisz, D., Christensen, C., Freeman, K., Gilbert, K., Gallart, C., Harris, J., Hodge, P., de Jong, R.S., Karachentseva, V., Mateo, M., Stetson, P.B., Tavarez, M., Zaritsky, D., Governato, F., Quinn, T.: Astrophys. J. Suppl. Ser. 183, 67 (2009)

Dale, D.A., Cohen, S.A., Johnson, L.C., Schuster, M.D., Calzetti, D., Engelbracht, C.W., Gil de Paz, A., Kennicutt, R.C., Lee, J.C., Begum, A., Block, M., Dalcanton, J.J., Funes, J.G., Gordon, K.D., Johnson, B.D., Marble, A.R., Sakai, S., Skillman, E.D., van Zee, L., Walter, F., Weisz, D.R., Williams, B., Wu, S., Wu, Y.: Astrophys. J. 703, 517 (2009)

Dale, D.A., Gil de Paz, A., Gordon, K.D., Hanson, H.M., Armus, L., Bendo, G.J., Bianchi, L., Block, M., Boissier, S., Boselli, A., Buckalew, B.A., Buat, V., Burgarella, D., Calzetti, D., Cannon, J.M., Engelbracht, C.W., Helou, G., Hollenbach, D.J., Jarrett, T.H., Kennicutt, R.C., Leitherer, C., Li, A., Madore, B.F., Martin, D.C., Meyer, M.J., Murphy, E.J., Regan, M.W., Roussel, H., Smith, J.D.T., Sosey, M.L., Thilker, D.A., Walter, F.: Astrophys. J. 655, 863 (2007)
Dale, D.A., Helou, G.: Astrophys. J. 576, 159 (2002)

Dale, D.A., Helou, G., Contursi, A., Silbermann, N.A., Kolhatkar, S.: Astrophys. J. 549, 215 (2001)

Davis, M., Guhathakurta, P., Konidaris, N.P., Newman, J.A., Ashby, M.L.N., Biggs, A.D., Barmby, P., Bundy, K., Chapman, S.C., Coil, A.L., Conselice, C.J., Cooper, M.C., Croton, D.J., Eisenhardt, P.R.M., Ellis, R.S., Faber, S.M., Fang, T., Fazio, G.G., Georgakakis, A., Gerke, B.F., Goss, W.M., Gwyn, S., Harker, J., Hopkins, A.M., Huang, J., Ivison, R.J., Kassin, S.A., Kirby, E.N., Koekemoer, A.M., Koo, D.C., Laird, E.S., Le Floc'h, E., Lin, L., Lotz, J.M., Marshall, P.J., Martin, D.C., Metevier, A.J., Moustakas, L.A., Nandra, K., Noeske, K.G., Papovich, C., Phillips, A.C., Rich, R.M., Rieke, G.H., Rigopoulou, D., Salim, S., Schiminovich, D., Simard, L., Smail, I., Small, T.A., Weiner, B.J., Willmer, C.N.A., Willner, S.P., Wilson, G., Wright, E.L., Yan, R.: Astrophys. J. Lett. 660, L1 (2007)

De Lucia, G., Blaizot, J.: Mon. Not. R. Astron. Soc. 375, 2 (2007)

De Lucia, G., Kauffmann, G., White, S.D.M.: Mon. Not. R. Astron. Soc. 349, 1101 (2004)

Demarque, P., Woo, J.H., Kim, Y.C., Yi, S.K.: Astrophys. J. Suppl. Ser. 155, 667 (2004)

Desert, F.X., Boulanger, F., Puget, J.L.: Astron. Astrophys. 237, 215 (1990)

Devriendt, J.E.G., Guiderdoni, B.: Astron. Astrophys. 363, 851 (2000)

Devriendt, J.E.G., Guiderdoni, B., Sadat, R.: Astron. Astrophys. 350, 381 (1999)

Dolag, K., Borgani, S., Schindler, S., Diaferio, A., Bykov, A.M.: Space Science Reviews 134, 229 (2008)

Dopita, M.A., Fischera, J., Sutherland, R.S., Kewley, L.J., Leitherer, C., Tuffs, R.J., Popescu, C.C., van Breugel, W., Groves, B.A.: Astrophys. J. Suppl. Ser. 167, 177 (2006b)

Dopita, M.A., Fischera, J., Sutherland, R.S., Kewley, L.J., Tuffs, R.J., Popescu, C.C., van Breugel, W., Groves, B.A., Leitherer, C.: Astrophys. J. 647, 244 (2006a)

Dopita, M.A., Groves, B.A., Fischera, J., Sutherland, R.S., Tuffs, R.J., Popescu, C.C., Kewley, L.J., Reuland, M., Leitherer, C.: Astrophys. J. 619, 755 (2005)

Dopita, M.A., Groves, B.A., Sutherland, R.S., Binette, L., Cecil, G.: Astrophys. J. 572, 753 (2002)

Dotter, A., Chaboyer, B., Ferguson, J.W., Lee, H.c., Worthey, G., Jevremović, D., Baron, E.: Astrophys. J. 666, 403 (2007)

Draine, B.T.: ArXiv e-prints (2010, in press)

Draine, B.T., Dale, D.A., Bendo, G., Gordon, K.D., Smith, J.D.T., Armus, L., Engelbracht, C.W., Helou, G., Kennicutt, R.C.Jr., Li, A., Roussel, H., Walter, F., Calzetti, D., Moustakas, J., Murphy, E.J., Rieke, G.H., Bot, C., Hollenbach, D.J., Sheth, K., Teplitz, H.I.: Astrophys. J. 663, 866 (2007b)

Draine, B.T., Lee, H.M.: Astrophys. J. 285, 89 (1984)

Draine, B.T., Li, A.: Astrophys. J. 657, 810 (2007a)

Draine, B.: Annu. Rev. Astron. Astrophys. 41(1), 241 (2003)

Driver, S.P., Norberg, P., Baldry, I.K., Bamford, S.P., Hopkins, A.M., Liske, J., Loveday, J., Peacock, J.A., Hill, D.T., Kelvin, L.S., Robotham, A.S.G., Cross, N.J.G., Parkinson, H.R., Prescott, M., Conselice, C.J., Dunne, L., Brough, S., Jones, H., Sharp, R.G., van Kampen, E., Oliver, S., Roseboom, I.G., Bland-Hawthorn, J., Croom, S.M., Ellis, S., Cameron, E., Cole, S., Frenk, C.S., Couch, W.J., Alister, W.G., Proctor, R., De Propris, R., Doyle, I.F., Edmondson, E.M., Nichol, R.C., Thomas, D., Eales, S.A., Jarvis, M.J., Kuijken, K., Lahav, O., 
Madore, B.F., Seibert, M., Meyer, M.J., Staveley-Smith, L., Phillipps, S., Popescu, C.C., Sansom, A.E., Sutherland, W.J., Tuffs, R.J., Warren, S.J.: Astronomy and Geophysics 50(5), 050000 (2009)

Drory, N., Bender, R., Hopp, U.: Astrophys. J. Lett. 616, L103 (2004)

Dunne, L., Eales, S., Edmunds, M., Ivison, R., Alexander, P., Clements, D.L.: Mon. Not. R. Astron. Soc. 315, 115 (2000)

Dunne, L., Eales, S.A.: Mon. Not. R. Astron. Soc. 327, 697 (2001)

Dye, S.: Mon. Not. R. Astron. Soc. 389, 1293 (2008)

E. Corbelli, F. Palla, \& H. Zinnecker (ed.): The Initial Mass Function 50 years later. vol. 327. (2005)

Efstathiou, A., Rowan-Robinson, M., Siebenmorgen, R.: Mon. Not. R. Astron. Soc. 313, 734 (2000)

Elíasdóttir, Á., Fynbo, J.P.U., Hjorth, J., Ledoux, C., Watson, D.J., Andersen, A.C., Malesani, D., Vreeswijk, P.M., Prochaska, J.X., Sollerman, J., Jaunsen, A.O.: Astrophys. J. 697, 1725 (2009)

Faber, S.M., Friel, E.D., Burstein, D., Gaskell, C.M.: Astrophys. J. Suppl. Ser. 57, 711 (1985)

Feldmann, R., Carollo, C.M., Porciani, C., Lilly, S.J., Capak, P., Taniguchi, Y., Le Fèvre, O., Renzini, A., Scoville, N., Ajiki, M., Aussel, H., Contini, T., McCracken, H., Mobasher, B., Murayama, T., Sanders, D., Sasaki, S., Scarlata, C., Scodeggio, M., Shioya, Y., Silverman, J., Takahashi, M., Thompson, D., Zamorani, G.: Mon. Not. R. Astron. Soc. 372, 565 (2006)

Ferland, G.J.: Annu. Rev. Astron. Astrophys. 41, 517 (2003)

Ferland, G.J., Korista, K.T., Verner, D.A., Ferguson, J.W., Kingdon, J.B., Verner, E.M.: Publ. Astron. Soc. Pac. 110, 761 (1998)

Ferrara, A., Bianchi, S., Cimatti, A., Giovanardi, C.: Astrophys. J. Suppl. Ser. 123, 437 (1999)

Ferraro, F.R., Sabbi, E., Gratton, R., Piotto, G., Lanzoni, B., Carretta, E., Rood, R.T., Sills, A., Fusi Pecci, F., Moehler, S., Beccari, G., Lucatello, S., Compagni, N.: Astrophys. J. Lett. 647, L53 (2006)

Ferreras, I., Silk, J.: Astrophys. J. Lett. 541, L37 (2000)

Fioc, M., Rocca-Volmerange, B.: Astron. Astrophys. 326, 950 (1997)

Fioc, M., Rocca-Volmerange, B.: ArXiv Astrophysics e-prints (1999, in press)

Firth, A.E., Lahav, O., Somerville, R.S.: Mon. Not. R. Astron. Soc. 339, 1195 (2003)

Fischera, J., Dopita, M.A., Sutherland, R.S.: Astrophys. J. Lett. 599, L21 (2003)

Fontanot, F., Somerville, R.S., Silva, L., Monaco, P., Skibba, R.: Mon. Not. R. Astron. Soc. 392, 553 (2009)

Förster Schreiber, N.M., van Dokkum, P.G., Franx, M., Labbé, I., Rudnick, G., Daddi, E., Illingworth, G.D., Kriek, M., Moorwood, A.F.M., Rix, H., Röttgering, H., Trujillo, I., van der Werf, P., van Starkenburg, L., Wuyts, S.: Astrophys. J. 616, 40 (2004)

Gallazzi, A., Bell, E.F.: Astrophys. J. Suppl. Ser. 185, 253 (2009)

Gallazzi, A., Brinchmann, J., Charlot, S., White, S.D.M.: Mon. Not. R. Astron. Soc. 383, 1439 (2008)

Gavazzi, G., Boselli, A., Donati, A., Franzetti, P., Scodeggio, M.: Astron. Astrophys. 400, 451 (2003)

Gerola, H., Seiden, P.E., Schulman, L.S.: Astrophys. J. 242, 517 (1980)
Giavalisco, M., Ferguson, H.C., Koekemoer, A.M., Dickinson, M., Alexander, D.M., Bauer, F.E., Bergeron, J., Biagetti, C., Brandt, W.N., Casertano, S., Cesarsky, C., Chatzichristou, E., Conselice, C., Cristiani, S., Da Costa, L., Dahlen, T., de Mello, D., Eisenhardt, P., Erben, T., Fall, S.M., Fassnacht, C., Fosbury, R., Fruchter, A., Gardner, J.P., Grogin, N., Hook, R.N., Hornschemeier, A.E., Idzi, R., Jogee, S., Kretchmer, C., Laidler, V., Lee, K.S., Livio, M., Lucas, R., Madau, P., Mobasher, B., Moustakas, L.A., Nonino, M., Padovani, P., Papovich, C., Park, Y., Ravindranath, S., Renzini, A., Richardson, M., Riess, A., Rosati, P., Schirmer, M., Schreier, E., Somerville, R.S., Spinrad, H., Stern, D., Stiavelli, M., Strolger, L., Urry, C.M., Vandame, B., Williams, R., Wolf, C.: Astrophys. J. Lett. 600, L93 (2004)

Gil de Paz, A., Boissier, S., Madore, B.F., Seibert, M., Joe, Y.H., Boselli, A., Wyder, T.K., Thilker, D., Bianchi, L., Rey, S.C., Rich, R.M., Barlow, T.A., Conrow, T., Forster, K., Friedman, P.G., Martin, D.C., Morrissey, P., Neff, S.G., Schiminovich, D., Small, T., Donas, J., Heckman, T.M., Lee, Y.W., Milliard, B., Szalay, A.S., Yi, S.: Astrophys. J. Suppl. Ser. 173, 185 (2007)

Girardi, L., Bressan, A., Bertelli, G., Chiosi, C.: Astron. Astrophys. Suppl. Ser. 141, 371 (2000)

Gómez, P.L., Nichol, R.C., Miller, C.J., Balogh, M.L., Goto, T., Zabludoff, A.I., Romer, A.K., Bernardi, M., Sheth, R., Hopkins, A.M., Castander, F.J., Connolly, A.J., Schneider, D.P., Brinkmann, J., Lamb, D.Q., SubbaRao, M., York, D.G.: Astrophys. J. 584, 210 (2003)

González Delgado, R.M., Cerviño, M., Martins, L.P., Leitherer, C., Hauschildt, P.H.: Mon. Not. R. Astron. Soc. 357, 945 (2005)

González Delgado, R.M., Cid Fernandes, R.: Mon. Not. R. Astron. Soc. 403, 797 (2010)

Gordon, K.D., Clayton, G.C.: Astrophys. J. 500, 816 (1998)

Gordon, K.D., Hanson, M.M., Clayton, G.C., Rieke, G.H., Misselt, K.A.: Astrophys. J. 519, 165 (1999)

Gordon, K.D., Misselt, K.A., Witt, A.N., Clayton, G.C.: Astrophys. J. 551, 269 (2001)

Granato, G.L., Lacey, C.G., Silva, L., Bressan, A., Baugh, C.M., Cole, S., Frenk, C.S.: Astrophys. J. 542, 710 (2000)

Graves, G.J., Faber, S.M., Schiavon, R.P.: Astrophys. J. 698, 1590 (2009)

Graves, G.J., Schiavon, R.P.: Astrophys. J. Suppl. Ser. 177, 446 (2008)

Gregg, M.D., Lacy, M., White, R.L., Glikman, E., Helfand, D., Becker, R.H., Brotherton, M.S.: Astrophys. J. 564, 133 (2002)

Gregg, M.D., Silva, D., Rayner, J., Valdes, F., Worthey, G., Pickles, A., Rose, J.A., Vacca, W., Carney, B.: In: Bulletin of the American Astronomical Society. Bulletin of the American Astronomical Society, vol. 36, 1496 (2004)

Groves, B., Dopita, M.A., Sutherland, R.S., Kewley, L.J., Fischera, J., Leitherer, C., Brandl, B., van Breugel, W.: Astrophys. J. Suppl. Ser. 176, 438 (2008)

Guhathakurta, P., Draine, B.T.: Astrophys. J. 345, 230 (1989)

Guiderdoni, B., Hivon, E., Bouchet, F.R., Maffei, B.: Mon. Not. R. Astron. Soc. 295, 877 (1998)

Guiderdoni, B., Rocca-Volmerange, B.: Astron. Astrophys. 186, 1 (1987)

Guillaume, M., Llebaria, A., Aymeric, D., Arnouts, S., Milliard, B.: In: Dougherty, E.R., Astola, J.T., Egiazarian, K.O., Nasrabadi, N.M., Rizvi, S.A. (eds.) Society of Photo-Optical Instrumentation Engineers (SPIE) Conference Series. Society of PhotoOptical Instrumentation Engineers (SPIE) Conference Series, vol. 6064,332 (2006) 
Gwyn, S.D.J., Hartwick, F.D.A.: Astrophys. J. Lett. 468, L77 (1996)

Han, Z., Podsiadlowski, P., Lynas-Gray, A.E.: Mon. Not. R. Astron. Soc. 380, 1098 (2007)

Hatton, S., Devriendt, J.E.G., Ninin, S., Bouchet, F.R., Guiderdoni, B., Vibert, D.: Mon. Not. R. Astron. Soc. 343, 75 (2003)

Hauser, M.G., Dwek, E.: Annu. Rev. Astron. Astrophys. 39, 249 (2001)

Heavens, A., Panter, B., Jimenez, R., Dunlop, J.: Nature 428, 625 (2004)

Heavens, A.F., Jimenez, R., Lahav, O.: Mon. Not. R. Astron. Soc. 317, 965 (2000)

Heber, U.: Memorie della Societa Astronomica Italiana 79, 375 (2008)

Hensler, G.: ArXiv e-prints (2008, in press)

Hippelein, H., Haas, M., Tuffs, R.J., Lemke, D., Stickel, M., Klaas, U., Völk, H.J.: Astron. Astrophys. 407, 137 (2003)

Hollenbach, D.J., Tielens, A.G.G.M.: Annu. Rev. Astron. Astrophys. 35, 179 (1997)

Iglesias-Páramo, J., Buat, V., Hernández-Fernández, J., Xu, C.K., Burgarella, D., Takeuchi, T.T., Boselli, A., Shupe, D., RowanRobinson, M., Babbedge, T., Conrow, T., Fang, F., Farrah, D., González-Solares, E., Lonsdale, C., Smith, G., Surace, J., Barlow, T.A., Forster, K., Friedman, P.G., Martin, D.C., Morrissey, P., Neff, S.G., Schiminovich, D., Seibert, M., Small, T., Wyder, T.K., Bianchi, L., Donas, J., Heckman, T.M., Lee, Y., Madore, B.F., Milliard, B., Rich, R.M., Szalay, A.S., Welsh, B.Y., Yi, S.K.: Astrophys. J. 670, 279 (2007)

Ilbert, O., Arnouts, S., McCracken, H.J., Bolzonella, M., Bertin, E., Le Fèvre, O., Mellier, Y., Zamorani, G., Pellò, R., Iovino, A., Tresse, L., Le Brun, V., Bottini, D., Garilli, B., Maccagni, D., Picat, J.P., Scaramella, R., Scodeggio, M., Vettolani, G., Zanichelli, A., Adami, C., Bardelli, S., Cappi, A., Charlot, S., Ciliegi, P., Contini, T., Cucciati, O., Foucaud, S., Franzetti, P., Gavignaud, I., Guzzo, L., Marano, B., Marinoni, C., Mazure, A., Meneux, B., Merighi, R., Paltani, S., Pollo, A., Pozzetti, L., Radovich, M., Zucca, E., Bondi, M., Bongiorno, A., Busarello, G., de La Torre, S., Gregorini, L., Lamareille, F., Mathez, G., Merluzzi, P., Ripepi, V., Rizzo, D., Vergani, D.: Astron. Astrophys. 457, 841 (2006)

Ilbert, O., Capak, P., Salvato, M., Aussel, H., McCracken, H.J., Sanders, D.B., Scoville, N., Kartaltepe, J., Arnouts, S., Floc'h, E.L., Mobasher, B., Taniguchi, Y., Lamareille, F., Leauthaud, A., Sasaki, S., Thompson, D., Zamojski, M., Zamorani, G., Bardelli, S., Bolzonella, M., Bongiorno, A., Brusa, M., Caputi, K.I., Carollo, C.M., Contini, T., Cook, R., Coppa, G., Cucciati, O., de la Torre, S., de Ravel, L., Franzetti, P., Garilli, B., Hasinger, G., Iovino, A., Kampczyk, P., Kneib, J.P., Knobel, C., Kovac, K., LeBorgne, J.F., LeBrun, V., Fèvre, O.L., Lilly, S., Looper, D., Maier, C., Mainieri, V., Mellier, Y., Mignoli, M., Murayama, T., Pellò, R., Peng, Y., PérezMontero, E., Renzini, A., Ricciardelli, E., Schiminovich, D., Scodeggio, M., Shioya, Y., Silverman, J., Surace, J., Tanaka, M., Tasca, L., Tresse, L., Vergani, D., Zucca, E.: Astrophys. J. 690, 1236 (2009a)

Ilbert, O., Salvato, M., Le Floc'h, E., Aussel, H., Capak, P., McCracken, H.J., Mobasher, B., Kartaltepe, J., Scoville, N., Sanders, D.B., Arnouts, S., Bundy, K., Cassata, P., Kneib, J., Koekemoer, A., Le Fèvre, O., Lilly, S., Surace, J., Taniguchi, Y., Tasca, L., Thompson, D., Tresse, L., Zamojski, M., Zamorani, G., Zucca, E.: Astrophys. J. 709, 644 (2010)
Inoue, A.K., Buat, V., Burgarella, D., Panuzzo, P., Takeuchi, T.T., Iglesias-Páramo, J.: Mon. Not. R. Astron. Soc. 370, 380 (2006)

Israel, F.P., Wall, W.F., Raban, D., Reach, W.T., Bot, C., Oonk, J.B.R., Ysard, N., Bernard, J.P.: ArXiv e-prints (2010, in press)

Johnson, B.D., Schiminovich, D., Seibert, M., Treyer, M., Martin, D.C., Barlow, T.A., Forster, K., Friedman, P.G., Morrissey, P., Neff, S.G., Small, T., Wyder, T.K., Bianchi, L., Donas, J., Heckman, T.M., Lee, Y.W., Madore, B.F., Milliard, B., Rich, R.M., Szalay, A.S., Welsh, B.Y., Yi, S.K.: Astrophys. J. Suppl. Ser. 173, 392 (2007a)

Johnson, B.D., Schiminovich, D., Seibert, M., Treyer, M.A., Charlot, S., Heckman, T.M., Martin, D.C., Salim, S., Kauffmann, G., Bianchi, L., Donas, J., Friedman, P.G., Lee, Y.W., Madore, B.F., Milliard, B., Morrissey, P., Neff, S.G., Rich, R.M., Szalay, A.S., Forster, K., Barlow, T.A., Conrow, T., Small, T., Wyder, T.K.: Astrophys. J. Lett. 644, L109 (2006)

Jones, A.P., Tielens, A.G.G.M., Hollenbach, D.J.: Astrophys. J. 469, 740 (1996)

Jonsson, P.: Mon. Not. R. Astron. Soc. 372, 2 (2006)

Jonsson, P., Groves, B.A., Cox, T.J.: Mon. Not. R. Astron. Soc. 403, 17 (2010)

Juvela, M.: Astron. Astrophys. 440, 531 (2005)

Kajisawa, M., Ichikawa, T., Tanaka, I., Konishi, M., Yamada, T., Akiyama, M., Suzuki, R., Tokoku, C., Uchimoto, Y.K., Yoshikawa, T., Ouchi, M., Iwata, I., Hamana, T., Onodera, M.: ArXiv e-prints (2009, in press)

Kakazu, Y., Cowie, L.L., Hu, E.M.: Astrophys. J. 668, 853 (2007)

Kauffmann, G., Colberg, J.M., Diaferio, A., White, S.D.M.: Mon. Not. R. Astron. Soc. 303, 188 (1999)

Kauffmann, G., Haehnelt, M.: Mon. Not. R. Astron. Soc. 311, 576 (2000)

Kauffmann, G., Heckman, T.M., Tremonti, C., Brinchmann, J., Charlot, S., White, S.D.M., Ridgway, S.E., Brinkmann, J., Fukugita, M., Hall, P.B., Ivezić, Ž., Richards, G.T., Schneider, D.P.: Mon. Not. R. Astron. Soc. 346, 1055 (2003b)

Kauffmann, G., Heckman, T.M., White, S.D.M., Charlot, S., Tremonti, C., Brinchmann, J., Bruzual, G., Peng, E.W., Seibert, M., Bernardi, M., Blanton, M., Brinkmann, J., Castander, F., Csábai, I., Fukugita, M., Ivezic, Z., Munn, J.A., Nichol, R.C., Padmanabhan, N., Thakar, A.R., Weinberg, D.H., York, D.: Mon. Not. R. Astron. Soc. 341, 33 (2003a)

Kaviani, A., Haehnelt, M.G., Kauffmann, G.: Mon. Not. R. Astron. Soc. 340, 739 (2003)

Kaviraj, S., Schawinski, K., Devriendt, J.E.G., Ferreras, I., Khochfar, S., Yoon, S.J., Yi, S.K., Deharveng, J.M., Boselli, A., Barlow, T., Conrow, T., Forster, K., Friedman, P.G., Martin, D.C., Morrissey, P., Neff, S., Schiminovich, D., Seibert, M., Small, T., Wyder, T., Bianchi, L., Donas, J., Heckman, T., Lee, Y.W., Madore, B., Milliard, B., Rich, R.M., Szalay, A.: Astrophys. J. Suppl. Ser. 173, 619 (2007)

Keel, W.C.: Astron. J. 131, 2755 (2006)

Kelson, D.D., Holden, B.P.: ArXiv e-prints (2010, in press)

Kennicutt, R.C., Hao, C., Calzetti, D., Moustakas, J., Dale, D.A., Bendo, G., Engelbracht, C.W., Johnson, B.D., Lee, J.C.: Astrophys. J. 703, 1672 (2009)

Kennicutt, R.C.Jr.: Annu. Rev. Astron. Astrophys. 36, 189 (1998)

Kennicutt, R.C.Jr., Armus, L., Bendo, G., Calzetti, D., Dale, D.A., Draine, B.T., Engelbracht, C.W., Gordon, K.D., Grauer, A.D., Helou, G., Hollenbach, D.J., Jarrett, T.H., Kewley, L.J., Leitherer, C., Li, A., Malhotra, S., Regan, M.W., Rieke, G.H., Rieke, M.J., Roussel, H., Smith, J.D.T., Thornley, M.D., Walter, F.: Publ. Astron. Soc. Pac. 115, 928 (2003) 
Kennicutt, R.C.Jr., Lee, J.C., Funes, S.J.José G., Sakai, S., Akiyama, S.: Astrophys. J. Suppl. Ser. 178, 247 (2008)

Kewley, L.J., Dopita, M.A., Sutherland, R.S., Heisler, C.A., Trevena, J.: Astrophys. J. 556, 121 (2001)

Kinney, A.L., Calzetti, D., Bohlin, R.C., McQuade, K., StorchiBergmann, T., Schmitt, H.R.: Astrophys. J. 467, 38 (1996)

Klaas, U., Haas, M., Müller, S.A.H., Chini, R., Schulz, B., Coulson, I., Hippelein, H., Wilke, K., Albrecht, M., Lemke, D.: Astron. Astrophys. 379, 823 (2001)

Koleva, M., Prugniel, P., Bouchard, A., Wu, Y.: ArXiv e-prints (2009, in press)

Koleva, M., Prugniel, P., Ocvirk, P., Le Borgne, D., Soubiran, C.: Mon. Not. R. Astron. Soc. 385, 1998 (2008)

Kong, X., Charlot, S., Brinchmann, J., Fall, S.M.: Mon. Not. R. Astron. Soc. 349, 769 (2004)

Koo, D.C.: Astron. J. 90, 418 (1985)

Koo, D.C.: In: Weymann, R., Storrie-Lombardi, L., Sawicki, M., Brunner, R. (eds.) Photometric Redshifts and the Detection of High Redshift Galaxies. Astronomical Society of the Pacific Conference Series, vol. 191, 3 (1999)

Koornneef, J., Bohlin, R., Buser, R., Horne, K., Turnshek, D.: Highlights of Astronomy 7, 833 (1986)

Kriek, M., van der Wel, A., van Dokkum, P.G., Franx, M., Illingworth, G.D.: Astrophys. J. 682, 896 (2008b)

Kriek, M., van Dokkum, P.G., Franx, M., Illingworth, G.D., Marchesini, D., Quadri, R., Rudnick, G., Taylor, E.N., Förster Schreiber, N.M., Gawiser, E., Labbé, I., Lira, P., Wuyts, S.: Astrophys. J. 677, 219 (2008)

Kriek, M., van Dokkum, P.G., Labbé, I., Franx, M., Illingworth, G.D., Marchesini, D., Quadri, R.F.: Astrophys. J. 700, 221 (2009a)

Kroupa, P.: Mon. Not. R. Astron. Soc. 322, 231 (2001)

Krugel, E., Siebenmorgen, R.: Astron. Astrophys. 282, 407 (1994)

Kuchinski, L.E., Terndrup, D.M., Gordon, K.D., Witt, A.N.: Astron. J. 115, 1438 (1998)

Kurtz, M.J., Geller, M.J., Fabricant, D.G., Wyatt, W.F., Dell'Antonio, I.P.: Astron. J. 134, 1360 (2007)

Kurucz, R.L.: In: Barbuy, B., Renzini, A. (eds.) The Stellar Populations of Galaxies. IAU Symposium, vol. 149, 225 (1992)

Kurucz, R.L.: Memorie della Societa Astronomica Italiana Supplement 8, 86 (2005)

Kylafis, N.D., Bahcall, J.N.: Astrophys. J. 317, 637 (1987)

Lacey, C.G., Baugh, C.M., Frenk, C.S., Silva, L., Granato, G.L., Bressan, A.: Mon. Not. R. Astron. Soc. 385, 1155 (2008)

Lagache, G., Dole, H., Puget, J.L., Pérez-González, P.G., Le Floc'h, E., Rieke, G.H., Papovich, C., Egami, E., AlonsoHerrero, A., Engelbracht, C.W., Gordon, K.D., Misselt, K.A., Morrison, J.E.: Astrophys. J. Suppl. Ser. 154, 112 (2004)

Lamareille, F.e.a.: Astron. Astrophys. 495, 53 (2009)

Lançon, A., Gallagher, J.S.III, Mouhcine, M., Smith, L.J., Ladjal, D., de Grijs, R.: Astron. Astrophys. 486, 165 (2008)

Lançon, A., Hauschildt, P.H., Ladjal, D., Mouhcine, M.: Astron. Astrophys. 468, 205 (2007)

Lançon, A., Mouhcine, M.: In: Lançon, A., Boily, C.M. (eds.) Massive Stellar Clusters. Astronomical Society of the Pacific Conference Series, vol. 211, 34 (2000a)

Landolt, A.U.: Astron. J. 104, 340 (1992)

Lanzetta, K.M., Yahil, A., Fernández-Soto, A.: Nature 381, 759 (1996)

Larson, R.B., Tinsley, B.M.: Astrophys. J. 219, 46 (1978)
Le Borgne, D., Rocca-Volmerange, B., Prugniel, P., Lançon, A., Fioc, M., Soubiran, C.: Astron. Astrophys. 425, 881 (2004)

Le Borgne, J.F., Bruzual, G., Pelló, R., Lançon, A., RoccaVolmerange, B., Sanahuja, B., Schaerer, D., Soubiran, C., Vílchez-Gómez, R.: Astron. Astrophys. 402, 433 (2003)

Le Fèvre, O., Vettolani, G., Garilli, B., Tresse, L., Bottini, D., Le Brun, V., Maccagni, D., Picat, J.P., Scaramella, R., Scodeggio, M., Zanichelli, A., Adami, C., Arnaboldi, M., Arnouts, S., Bardelli, S., Bolzonella, M., Cappi, A., Charlot, S., Ciliegi, P., Contini, T., Foucaud, S., Franzetti, P., Gavignaud, I., Guzzo, L., Ilbert, O., Iovino, A., McCracken, H.J., Marano, B., Marinoni, C., Mathez, G., Mazure, A., Meneux, B., Merighi, R., Paltani, S., Pellò, R., Pollo, A., Pozzetti, L., Radovich, M., Zamorani, G., Zucca, E., Bondi, M., Bongiorno, A., Busarello, G., Lamareille, F., Mellier, Y., Merluzzi, P., Ripepi, V., Rizzo, D.: Astron. Astrophys. 439, 845 (2005)

Lee, H., Worthey, G., Dotter, A., Chaboyer, B., Jevremović, D., Baron, E., Briley, M.M., Ferguson, J.W., Coelho, P., Trager, S.C.: Astrophys. J. 694, 902 (2009b)

Lee, J.C., Gil de Paz, A., Tremonti, C., Kennicutt, R.C.Jr., Salim, S., Bothwell, M., Calzetti, D., Dalcanton, J., Dale, D., Engelbracht, C., Jose G. Funes S., J., Johnson, B., Sakai, S., Skillman, E., van Zee, L., Walter, F., Weisz, D.: ArXiv e-prints (2009a, in press)

Lee, Y.W., Joo, J.M., Sohn, Y.J., Rey, S.C., Lee, H.C., Walker, A.R.: Nature 402, 55 (1999)

Leger, A., Puget, J.L.: Astron. Astrophys. 137, L5 (1984)

Leitherer, C., Schaerer, D., Goldader, J.D., Delgado, R.M.G., Robert, C., Kune, D.F., de Mello, D.F., Devost, D., Heckman, T.M.: Astrophys. J. Suppl. Ser. 123, 3 (1999)

Lejeune, T., Schaerer, D.: Astron. Astrophys. 366, 538 (2001)

Lilly, S.J., Le Fèvre, O., Renzini, A., Zamorani, G., Scodeggio, M., Contini, T., Carollo, C.M., Hasinger, G., Kneib, J.P., Iovino, A., Le Brun, V., Maier, C., Mainieri, V., Mignoli, M., Silverman, J., Tasca, L.A.M., Bolzonella, M., Bongiorno, A., Bottini, D., Capak, P., Caputi, K., Cimatti, A., Cucciati, O., Daddi, E., Feldmann, R., Franzetti, P., Garilli, B., Guzzo, L., Ilbert, O., Kampczyk, P., Kovac, K., Lamareille, F., Leauthaud, A., Borgne, J.F.L., McCracken, H.J., Marinoni, C., Pello, R., Ricciardelli, E., Scarlata, C., Vergani, D., Sanders, D.B., Schinnerer, E., Scoville, N., Taniguchi, Y., Arnouts, S., Aussel, H., Bardelli, S., Brusa, M., Cappi, A., Ciliegi, P., Finoguenov, A., Foucaud, S., Franceschini, R., Halliday, C., Impey, C., Knobel, C., Koekemoer, A., Kurk, J., Maccagni, D., Maddox, S., Marano, B., Marconi, G., Meneux, B., Mobasher, B., Moreau, C., Peacock, J.A., Porciani, C., Pozzetti, L., Scaramella, R., Schiminovich, D., Shopbell, P., Smail, I., Thompson, D., Tresse, L., Vettolani, G., Zanichelli, A., Zucca, E.: Astrophys. J. Suppl. Ser. 172, 70 (2007)

Lisenfeld, U., Israel, F.P., Stil, J.M., Sievers, A.: Astron. Astrophys. 382, 860 (2002)

Longhetti, M., Saracco, P.: Mon. Not. R. Astron. Soc. 394, 774 (2009)

Lonsdale, C.J., Smith, H.E., Rowan-Robinson, M., Surace, J., Shupe, D., Xu, C., Oliver, S., Padgett, D., Fang, F., Conrow, T., Franceschini, A., Gautier, N., Griffin, M., Hacking, P., Masci, F., Morrison, G., O’Linger, J., Owen, F., PérezFournon, I., Pierre, M., Puetter, R., Stacey, G., Castro, S., Polletta, M.d.C., Farrah, D., Jarrett, T., Frayer, D., Siana, B., Babbedge, T., Dye, S., Fox, M., Gonzalez-Solares, E., Salaman, M., Berta, S., Condon, J.J., Dole, H., Serjeant, S.: Publ. Astron. Soc. Pac. 115, 897 (2003) 
Maeder, A., Meynet, G.: Astron. Astrophys. Suppl. Ser. 76, 411 (1988)

Magnelli, B., Elbaz, D., Chary, R.R., Dickinson, M., Le Borgne, D., Frayer, D.T., Willmer, C.N.A.: Astron. Astrophys. 496, 57 (2009)

Mandelbaum, R., Hirata, C.M., Seljak, U., Guzik, J., Padmanabhan, N., Blake, C., Blanton, M.R., Lupton, R., Brinkmann, J.: Mon. Not. R. Astron. Soc. 361, 1287 (2005)

Maraston, C.: Mon. Not. R. Astron. Soc. 300, 872 (1998)

Maraston, C.: Mon. Not. R. Astron. Soc. 362, 799 (2005)

Maraston, C., Nieves Colmenárez, L., Bender, R., Thomas, D.: Astron. Astrophys. 493, 425 (2009)

Margoniner, V.E., Wittman, D.M.: Astrophys. J. 679, 31 (2008)

Marigo, P., Girardi, L.: Astron. Astrophys. 469, 239 (2007)

Marigo, P., Girardi, L., Bressan, A., Groenewegen, M.A.T., Silva, L., Granato, G.L.: Astron. Astrophys. 482, 883 (2008)

Marshall, J.A., Herter, T.L., Armus, L., Charmandaris, V., Spoon, H.W.W., Bernard-Salas, J., Houck, J.R.: Astrophys. J. 670, 129 (2007)

Martins, L.P., Coelho, P.: Mon. Not. R. Astron. Soc. 381, 1329 (2007)

Martins, L.P., Delgado, R.M.G., Leitherer, C., Cerviño, M., Hauschildt, P.: Mon. Not. R. Astron. Soc. 358, 49 (2005)

Massey, P.: In: Gilmore, G., Howell, D. (eds.) The Stellar Initial Mass Function (38th Herstmonceux Conference). Astronomical Society of the Pacific Conference Series, vol. 142, 17 (1998)

Mathis, J.S., Rumpl, W., Nordsieck, K.H.: Astrophys. J. 217, 425 (1977)

Matteucci, F.: ArXiv e-prints (2008, in press)

Mennella, V., Brucato, J.R., Colangeli, L., Palumbo, P., Rotundi, A., Bussoletti, E.: Astrophys. J. 496, 1058 (1998)

Meurer, G.R., Heckman, T.M., Calzetti, D.: Astrophys. J. 521, 64 (1999)

Meynet, G., Maeder, A.: Astron. Astrophys. 429, 581 (2005)

Misiriotis, A., Popescu, C.C., Tuffs, R., Kylafis, N.D.: Astron. Astrophys. 372, 775 (2001)

Misselt, K.A., Clayton, G.C., Gordon, K.D.: Astrophys. J. 515, 128 (1999)

Misselt, K.A., Gordon, K.D., Clayton, G.C., Wolff, M.J.: Astrophys. J. 551, 277 (2001)

Mollá, M., García-Vargas, M.L., Bressan, A.: Mon. Not. R. Astron. Soc. 398, 451 (2009)

Moore, S.A.W., Lucey, J.R., Kuntschner, H., Colless, M.: Mon. Not. R. Astron. Soc. 336, 382 (2002)

Muzzin, A., Marchesini, D., van Dokkum, P.G., Labbé, I., Kriek, M., Franx, M.: Astrophys. J. 701, 1839 (2009a)

Muzzin, A., van Dokkum, P., Franx, M., Marchesini, D., Kriek, M., Labbé, I.: Astrophys. J. Lett. 706, L188 (2009b)

Negrello, M., Serjeant, S., Pearson, C., Takagi, T., Efstathiou, A., Goto, T., Burgarella, D., Jeong, W., Im, M., Lee, H.M., Matsuhara, H., Oyabu, S., Wada, T., White, G.: Mon. Not. R. Astron. Soc. 394, 375 (2009)

Nolan, L.A., Harva, M.O., Kabán, A., Raychaudhury, S.: Mon. Not. R. Astron. Soc. 366, 321 (2006)

Noll, S., Burgarella, D., Giovannoli, E., Buat, V., Marcillac, D., Muñoz-Mateos, J.C.: Astron. Astrophys. 507, 1793 (2009)

Noll, S., Pierini, D., Pannella, M., Savaglio, S.: Astron. Astrophys. 472, 455 (2007)

Noterdaeme, P., Ledoux, C., Srianand, R., Petitjean, P., Lopez, S.: Astron. Astrophys. 503, 765 (2009)

Ocvirk, P.: Astrophys. J. 709, 88 (2010)
Ocvirk, P., Pichon, C., Lançon, A., Thiébaut, E.: Mon. Not. R. Astron. Soc. 365, 74 (2006)

Oemler, A.Jr.: Astrophys. J. 209, 693 (1976)

Oosterloo, T., van Gorkom, J.: Astron. Astrophys. 437, L19 (2005)

Papovich, C., Dickinson, M., Ferguson, H.C.: Astrophys. J. 559, 620 (2001)

Pappalardo, C., Lancon, A., Vollmer, B., Ocvirk, P., Boissier, S., Boselli, A.: ArXiv e-prints (2010, in press)

Pellerin, A., Finkelstein, S.L.: ArXiv e-prints (2009, in press)

Pello, R., Miralles, J.M., Le Borgne, J.F., Picat, J.P., Soucail, G., Bruzual, G.: Astron. Astrophys. 314, 73 (1996)

Percival, S.M., Salaris, M.: Astrophys. J. 703, 1123 (2009)

Pierini, D., Gordon, K.D., Witt, A.N., Madsen, G.J.: Astrophys. J. 617, 1022 (2004)

Pietrinferni, A., Cassisi, S., Salaris, M., Percival, S., Ferguson, J.W.: Astrophys. J. 697, 275 (2009)

Piskunov, A.E., Kharchenko, N.V., Schilbach, E., Röser, S., Scholz, R., Zinnecker, H.: Astron. Astrophys. 507, L5 (2009)

Polletta, M., Tajer, M., Maraschi, L., Trinchieri, G., Lonsdale, C.J., Chiappetti, L., Andreon, S., Pierre, M., Le Fèvre, O., Zamorani, G., Maccagni, D., Garcet, O., Surdej, J., Franceschini, A., Alloin, D., Shupe, D.L., Surace, J.A., Fang, F., RowanRobinson, M., Smith, H.E., Tresse, L.: Astrophys. J. 663, 81 (2007)

Popescu, C.C., Misiriotis, A., Kylafis, N.D., Tuffs, R.J., Fischera, J.: Astron. Astrophys. 362, 138 (2000)

Popescu, C.C., Tuffs, R.J.: Mon. Not. R. Astron. Soc. 335, L41 (2002)

Pozzetti, L., Bolzonella, M., Lamareille, F., Zamorani, G., Franzetti, P., Le Fèvre, O., Iovino, A., Temporin, S., Ilbert, O., Arnouts, S., Charlot, S., Brinchmann, J., Zucca, E., Tresse, L., Scodeggio, M., Guzzo, L., Bottini, D., Garilli, B., Le Brun, V., Maccagni, D., Picat, J.P., Scaramella, R., Vettolani, G., Zanichelli, A., Adami, C., Bardelli, S., Cappi, A., Ciliegi, P., Contini, T., Foucaud, S., Gavignaud, I., McCracken, H.J., Marano, B., Marinoni, C., Mazure, A., Meneux, B., Merighi, R., Paltani, S., Pellò, R., Pollo, A., Radovich, M., Bondi, M., Bongiorno, A., Cucciati, O., de la Torre, S., Gregorini, L., Mellier, Y., Merluzzi, P., Vergani, D., Walcher, C.J.: Astron. Astrophys. 474, 443 (2007)

Prather, M.J.: The effect of a Brans-Dicke cosmology upon stellar evolution and the evolution of galaxies. $\mathrm{PhD}$ thesis, AA(Yale Univ., New Haven, CT.) (1976)

Prugniel, P., Soubiran, C.: Astron. Astrophys. 369, 1048 (2001)

Prugniel, P., Soubiran, C., Koleva, M., Le Borgne, D.: ArXiv Astrophysics e-prints (2007, in press)

Quillen, A.C., Bland-Hawthorn, J.: Mon. Not. R. Astron. Soc. 386, 2227 (2008)

Renzini, A., Fusi Pecci, F.: Annu. Rev. Astron. Astrophys. 26, 199 (1988)

Richards, J.W., Freeman, P.E., Lee, A.B., Schafer, C.M.: Mon. Not. R. Astron. Soc. 399, 1044 (2009)

Rieke, G.H., Alonso-Herrero, A., Weiner, B.J., PérezGonzález, P.G., Blaylock, M., Donley, J.L., Marcillac, D.: Astrophys. J. 692, 556 (2009)

Rix, S.A., Pettini, M., Leitherer, C., Bresolin, F., Kudritzki, R.P., Steidel, C.C.: Astrophys. J. 615, 98 (2004)

Robert, C., Pellerin, A., Aloisi, A., Leitherer, C., Hoopes, C., Heckman, T.M.: Astrophys. J. Suppl. Ser. 144, 21 (2003)

Rogers, B., Ferreras, I., Lahav, O., Bernardi, M., Kaviraj, S., Yi, S.K.: Mon. Not. R. Astron. Soc. 382, 750 (2007) 
Rogers, B., Ferreras, I., Pasquali, A., Bernardi, M., Lahav, O., Kaviraj, S.: Mon. Not. R. Astron. Soc., 387 (2010b)

Rogers, B., Ferreras, I., Peletier, R., Silk, J.: Mon. Not. R. Astron. Soc. 402, 447 (2010a)

Rose, J.A.: Astron. J. 89, 1238 (1984)

Roseboom, I.G., Oliver, S., Parkinson, D., Vaccari, M.: Mon. Not. R. Astron. Soc. 400, 1062 (2009)

Rowan-Robinson, M.: Astrophys. J. Suppl. Ser. 44, 403 (1980)

Rowan-Robinson, M.: Mon. Not. R. Astron. Soc. 258, 787 (1992)

Rowan-Robinson, M., Crawford, J.: Mon. Not. R. Astron. Soc. 238, 523 (1989)

Rowan-Robinson, M., Efstathiou, A.: Mon. Not. R. Astron. Soc. 263, 675 (1993)

Salasnich, B., Girardi, L., Weiss, A., Chiosi, C.: Astron. Astrophys. 361, 1023 (2000)

Salim, S., Dickinson, M., Michael Rich, R., Charlot, S., Lee, J.C., Schiminovich, D., Pérez-González, P.G., Ashby, M.L.N., Papovich, C., Faber, S.M., Ivison, R.J., Frayer, D.T., Walton, J.M., Weiner, B.J., Chary, R., Bundy, K., Noeske, K., Koekemoer, A.M.: Astrophys. J. 700, 161 (2009)

Salim, S., Rich, R.M., Charlot, S., Brinchmann, J., Johnson, B.D., Schiminovich, D., Seibert, M., Mallery, R., Heckman, T.M., Forster, K., Friedman, P.G., Martin, D.C., Morrissey, P., Neff, S.G., Small, T., Wyder, T.K., Bianchi, L., Donas, J., Lee, Y.W., Madore, B.F., Milliard, B., Szalay, A.S., Welsh, B.Y., Yi, S.K.: Astrophys. J. Suppl. Ser. 173, 267 (2007)

Salpeter, E.E.: Astrophys. J. 121, 161 (1955)

Salucci, P., Yegorova, I.A., Drory, N.: Mon. Not. R. Astron. Soc. 388, 159 (2008)

Sánchez-Blázquez, P., Peletier, R.F., Jiménez-Vicente, J., Cardiel, N., Cenarro, A.J., Falcón-Barroso, J., Gorgas, J., Selam, S., Vazdekis, A.: Mon. Not. R. Astron. Soc. 371, 703 (2006)

Sanders, D.B., Salvato, M., Aussel, H., Ilbert, O., Scoville, N., Surace, J.A., Frayer, D.T., Sheth, K., Helou, G., Brooke, T., Bhattacharya, B., Yan, L., Kartaltepe, J.S., Barnes, J.E., Blain, A.W., Calzetti, D., Capak, P., Carilli, C., Carollo, C.M., Comastri, A., Daddi, E., Ellis, R.S., Elvis, M., Fall, S.M., Franceschini, A., Giavalisco, M., Hasinger, G., Impey, C., Koekemoer, A., Le Fèvre, O., Lilly, S., Liu, M.C., McCracken, H.J., Mobasher, B., Renzini, A., Rich, M., Schinnerer, E., Shopbell, P.L., Taniguchi, Y., Thompson, D.J., Urry, C.M., Williams, J.P.: Astrophys. J. Suppl. Ser. 172, 86 (2007)

Sarajedini, V.L., Green, R.F., Griffiths, R.E., Ratnatunga, K.: Astrophys. J. Suppl. Ser. 121, 417 (1999)

Sauvage, M., Tuffs, R.J., Popescu, C.C.: Space Science Reviews 119, 313 (2005)

Sawicki, M., Yee, H.K.C.: Astron. J. 115, 1329 (1998)

Sawicki, M.J., Lin, H., Yee, H.K.C.: Astron. J. 113, 1 (1997)

Schombert, J., Rakos, K.: Astrophys. J. 699, 1530 (2009)

Schulz, J., Fritze-v. Alvensleben, U., Möller, C.S., Fricke, K.J.: Astron. Astrophys. 392, 1 (2002)

Schurer, A., Calura, F., Silva, L., Pipino, A., Granato, G.L., Matteucci, F., Maiolino, R.: ArXiv e-prints (2009, in press)

Scoville, N., Aussel, H., Brusa, M., Capak, P., Carollo, C.M., Elvis, M., Giavalisco, M., Guzzo, L., Hasinger, G., Impey, C., Kneib, J.P., LeFevre, O., Lilly, S.J., Mobasher, B., Renzini, A., Rich, R.M., Sanders, D.B., Schinnerer, E., Schminovich, D., Shopbell, P., Taniguchi, Y., Tyson, N.D.: Astrophys. J. Suppl. Ser. 172, 1 (2007)
Searle, L., Sargent, W.L.W., Bagnuolo, W.G.: Astrophys. J. 179, 427 (1973)

Seibert, M., Martin, D.C., Heckman, T.M., Buat, V., Hoopes, C., Barlow, T., Bianchi, L., Byun, Y.I., Donas, J., Forster, K., Friedman, P.G., Jelinsky, P., Lee, Y.W., Madore, B.F., Malina, R., Milliard, B., Morrissey, P., Neff, S., Rich, R.M., Schiminovich, D., Siegmund, O., Small, T., Szalay, A.S., Welsh, B., Wyder, T.K.: Astrophys. J. Lett. 619, 55 (2005)

Serra, P., Trager, S.C.: Mon. Not. R. Astron. Soc. 374, 769 (2007)

Siebenmorgen, R.: Astrophys. J. 408, 218 (1993)

Siebenmorgen, R., Kruegel, E.: Astron. Astrophys. 259, 614 (1992a)

Siebenmorgen, R., Kruegel, E., Mathis, J.S.: Astron. Astrophys. 266, 501 (1992b)

Siebenmorgen, R., Krügel, E.: Astron. Astrophys. 461, 445 (2007)

Silva, L., Granato, G.L., Bressan, A., Danese, L.: Astrophys. J. 509, 103 (1998)

Silva, L., Schurer, A., Granato, G.L., Almeida, C., Baugh, C.M., Frenk, C.S., Lacey, C.G., Paoletti, L., Petrella, A., Selvestrel, D.: ArXiv e-prints (2010, in press)

Smith, J.D.T., Draine, B.T., Dale, D.A., Moustakas, J., Kennicutt, R.C.Jr., Helou, G., Armus, L., Roussel, H., Sheth, K., Bendo, G.J., Buckalew, B.A., Calzetti, D., Engelbracht, C.W., Gordon, K.D., Hollenbach, D.J., Li, A., Malhotra, S., Murphy, E.J., Walter, F.: Astrophys. J. 656, 770 (2007)

Smith, L.J., Norris, R.P.F., Crowther, P.A.: Mon. Not. R. Astron. Soc. 337, 1309 (2002)

Somerville, R.S., Hopkins, P.F., Cox, T.J., Robertson, B.E., Hernquist, L.: Mon. Not. R. Astron. Soc. 391, 481 (2008)

Spoon, H.W.W., Marshall, J.A., Houck, J.R., Elitzur, M., Hao, L., Armus, L., Brandl, B.R., Charmandaris, V.: Astrophys. J. Lett. 654, L49 (2007)

Springel, V.: Mon. Not. R. Astron. Soc. 364, 1105 (2005)

Stasińska, G.: ArXiv e-prints (2007, in press)

Storey, P.J., Hummer, D.G.: Mon. Not. R. Astron. Soc. 272, 41 (1995)

Taylor, E.N., Franx, M., van Dokkum, P.G., Quadri, R.F., Gawiser, E., Bell, E.F., Barrientos, L.F., Blanc, G.A., Castander, F.J., Damen, M., Gonzalez-Perez, V., Hall, P.B., Herrera, D., Hildebrandt, H., Kriek, M., Labbé, I., Lira, P., Maza, J., Rudnick, G., Treister, E., Urry, C.M., Willis, J.P., Wuyts, S.: Astrophys. J. Suppl. Ser. 183, 295 (2009)

Thomas, D., Maraston, C., Bender, R., Mendes de Oliveira, C.: Astrophys. J. 621, 673 (2005)

Thomas, D., Maraston, C., Korn, A.: Mon. Not. R. Astron. Soc. 351, L19 (2004)

Tian, B., Deng, L., Han, Z., Zhang, X.B.: Astron. Astrophys. 455, 247 (2006)

Tinsley, B.M.: Astron. Astrophys. 20, 383 (1972)

Tojeiro, R., Heavens, A.F., Jimenez, R., Panter, B.: Mon. Not. R. Astron. Soc. 381, 1252 (2007)

Tojeiro, R., Wilkins, S., Heavens, A.F., Panter, B., Jimenez, R.: Astrophys. J. Suppl. Ser. 185, 1 (2009)

Tormen, G.: In: Ansari, R., Giraud-Heraud, Y., Tran Thanh van, J. (eds.) Dark Matter in Cosmology Quantam Measurements Experimental Gravitation, 207 (1996)

Trager, S.C., Faber, S.M., Dressler, A.: Mon. Not. R. Astron. Soc. 386, 715 (2008)

Trager, S.C., Faber, S.M., Worthey, G., González, J.J.: Astron. J. 120, 165 (2000)

Trager, S.C., Somerville, R.S.: ArXiv e-prints (2009, in press) 
Trager, S.C., Worthey, G., Faber, S.M., Burstein, D., Gonzalez, J.J.: Astrophys. J. Suppl. Ser. 116, 1 (1998)

Treffers, R., Cohen, M.: Astrophys. J. 188, 545 (1974)

Tremonti, C.A., Heckman, T.M., Kauffmann, G., Brinchmann, J., Charlot, S., White, S.D.M., Seibert, M., Peng, E.W., Schlegel, D.J., Uomoto, A., Fukugita, M., Brinkmann, J.: Astrophys. J. 613, 898 (2004)

Treyer, M., Schiminovich, D., Johnson, B.D., O’Dowd, M., Martin, C.D., Wyder, T., Charlot, S., Heckman, T., Martins, L., Seibert, M., van der Hulst, J.M.: ArXiv e-prints (2010, in press)

Tripicco, M.J., Bell, R.A.: Astron. J. 110, 3035 (1995)

Tsalmantza, P., Kontizas, M., Bailer-Jones, C.A.L., RoccaVolmerange, B., Korakitis, R., Kontizas, E., Livanou, E., Dapergolas, A., Bellas-Velidis, I., Vallenari, A., Fioc, M.: Astron. Astrophys. 470, 761 (2007)

Tsalmantza, P., Kontizas, M., Rocca-Volmerange, B., BailerJones, C.A.L., Kontizas, E., Bellas-Velidis, I., Livanou, E., Korakitis, R., Dapergolas, A., Vallenari, A., Fioc, M.: Astron. Astrophys. 504, 1071 (2009)

Tuffs, R.J., Popescu, C.C., Völk, H.J., Kylafis, N.D., Dopita, M.A.: Astron. Astrophys. 419, 821 (2004)

Valdes, F., Gupta, R., Rose, J.A., Singh, H.P., Bell, D.J.: Astrophys. J. Suppl. Ser. 152, 251 (2004)

van der Wel, A., Franx, M., Wuyts, S., van Dokkum, P.G., Huang, J., Rix, H., Illingworth, G.D.: Astrophys. J. 652, 97 (2006)

van Dokkum, P.G., Labbé, I., Marchesini, D., Quadri, R., Brammer, G., Whitaker, K.E., Kriek, M., Franx, M., Rudnick, G., Illingworth, G., Lee, K., Muzzin, A.: Publ. Astron. Soc. Pac. 121, 2 (2009)

van Dokkum, P.G., Quadri, R., Marchesini, D., Rudnick, G., Franx, M., Gawiser, E., Herrera, D., Wuyts, S., Lira, P., Labbé, I., Maza, J., Illingworth, G.D., Förster Schreiber, N.M., Kriek, M., Rix, H., Taylor, E.N., Toft, S., Webb, T., Yi, S.K.: Astrophys. J. Lett. 638, L59 (2006)

Vassiliadis, E., Wood, P.R.: Astrophys. J. 413, 641 (1993)

Vazdekis, A.: Astrophys. J. 513, 224 (1999)

Vázquez, G.A., Leitherer, C.: Astrophys. J. 621, 695 (2005)

Vázquez, G.A., Leitherer, C., Schaerer, D., Meynet, G., Maeder, A.: Astrophys. J. 663, 995 (2007)

Vlahakis, C., Dunne, L., Eales, S.: Mon. Not. R. Astron. Soc. 364, 1253 (2005)

Vollmer, B., Huchtmeier, W.: Astron. Astrophys. 406, 427 (2003)

Walcher, C.J., Böker, T., Charlot, S., Ho, L.C., Rix, H.W., Rossa, J., Shields, J.C., van der Marel, R.P.: Astrophys. J. 649, 692 (2006)

Walcher, C.J., Coelho, P., Gallazzi, A., Charlot, S.: Mon. Not. R. Astron. Soc., L275 (2009)

Walcher, C.J., Lamareille, F., Vergani, D., Arnouts, S., Buat, V., Charlot, S., Tresse, L., Le Fèvre, O., Bolzonella, M., Brinchmann, J., Pozzetti, L., Zamorani, G., Bottini, D., Garilli, B., Le Brun, V., Maccagni, D., Milliard, B., Scaramella, R., Scodeggio, M., Vettolani, G., Zanichelli, A., Adami, C., Bardelli, S., Cappi, A., Ciliegi, P., Contini, T., Franzetti, P., Foucaud, S., Gavignaud, I., Guzzo, L., Ilbert, O., Iovino, A., McCracken, H.J., Marano, B., Marinoni, C., Mazure, A., Meneux, B., Merighi, R., Paltani, S., Pellò, R., Pollo, A., Radovich, M., Zucca, E., Lonsdale, C., Martin, C.: Astron. Astrophys. 491, 713 (2008)

Wang, J., Hall, P.B., Ge, J., Li, A., Schneider, D.P.: Astrophys. J. 609, 589 (2004)

Wang, Y., Bahcall, N., Turner, E.L.: Astron. J. 116, 2081 (1998)
Weingartner, J.C., Draine, B.T.: Astrophys. J. 548, 296 (2001)

Weinstein, M.A., Richards, G.T., Schneider, D.P., Younger, J.D., Strauss, M.A., Hall, P.B., Budavári, T., Gunn, J.E., York, D.G., Brinkmann, J.: Astrophys. J. Suppl. Ser. 155, 243 (2004)

Weiss, A., Schlattl, H.: Astrophys. Space Sci. 316, 99 (2008)

Westera, P., Lejeune, T., Buser, R., Cuisinier, F., Bruzual, G.: Astron. Astrophys. 381, 524 (2002)

Wild, V., Kauffmann, G., Heckman, T., Charlot, S., Lemson, G., Brinchmann, J., Reichard, T., Pasquali, A.: Mon. Not. R. Astron. Soc. 381, 543 (2007)

Wild, V., Walcher, C.J., Johansson, P.H., Tresse, L., Charlot, S., Pollo, A., Le Fèvre, O., de Ravel, L.: Mon. Not. R. Astron. Soc. 395, 144 (2009)

Witt, A.N., Gordon, K.D.: Astrophys. J. 463, 681 (1996)

Witt, A.N., Gordon, K.D.: Astrophys. J. 528, 799 (2000)

Witt, A.N., Thronson, H.A.Jr., Capuano, J.M.Jr.: Astrophys. J. 393, $611(1992)$

Wittman, D.M., Tyson, J.A., Dell'Antonio, I.P., Becker, A., Margoniner, V., Cohen, J.G., Norman, D., Loomba, D., Squires, G., Wilson, G., Stubbs, C.W., Hennawi, J., Spergel, D.N., Boeshaar, P., Clocchiatti, A., Hamuy, M., Bernstein, G., Gonzalez, A., Guhathakurta, P., Hu, W., Seljak, U., Zaritsky, D.: In: Tyson, J.A., Wolff, S. (eds.) Society of Photo-Optical Instrumentation Engineers (SPIE) Conference Series. Society of PhotoOptical Instrumentation Engineers (SPIE) Conference Series, vol. 4836, 73 (2002)

Wolf, C., Meisenheimer, K., Rix, H., Borch, A., Dye, S., Kleinheinrich, M.: Astron. Astrophys. 401, 73 (2003)

Wolf, M.J., Drory, N., Gebhardt, K., Hill, G.J.: Astrophys. J. 655, 179 (2007)

Worthey, G., Faber, S.M., Gonzalez, J.J., Burstein, D.: Astrophys. J. Suppl. Ser. 94, 687 (1994)

Wray, J.J., Gunn, J.E.: Astrophys. J. 678, 144 (2008)

Wu, H., Cao, C., Hao, C., Liu, F., Wang, J., Xia, X., Deng, Z., Young, C.: Astrophys. J. Lett. 632, L79 (2005)

Wuyts, S., Franx, M., Cox, T.J., Hernquist, L., Hopkins, P.F., Robertson, B.E., van Dokkum, P.G.: Astrophys. J. 696, 348 (2009)

Xilouris, E.M., Alton, P.B., Davies, J.I., Kylafis, N.D., Papamastorakis, J., Trewhella, M.: Astron. Astrophys. 331, 894 (1998)

Xilouris, E.M., Byun, Y.I., Kylafis, N.D., Paleologou, E.V., Papamastorakis, J.: Astron. Astrophys. 344, 868 (1999)

Xin, Y., Deng, L., Han, Z.W.: Astrophys. J. 660, 319 (2007)

Yamada, Y., Arimoto, N., Vazdekis, A., Peletier, R.F.: Astrophys. J. 637, 200 (2006)

Yi, S.K., Yoon, S.J., Kaviraj, S., Deharveng, J.M., Rich, R.M., Salim, S., Boselli, A., Lee, Y.W., Ree, C.H., Sohn, Y.J., Rey, S.C., Lee, J.W., Rhee, J., Bianchi, L., Byun, Y.I., Donas, J., Friedman, P.G., Heckman, T.M., Jelinsky, P., Madore, B.F., Malina, R., Martin, D.C., Milliard, B., Morrissey, P., Neff, S., Schiminovich, D., Siegmund, O., Small, T., Szalay, A.S., Jee, M.J., Kim, S.W., Barlow, T., Forster, K., Welsh, B., Wyder, T.K.: Astrophys. J. Lett. 619, L111 (2005)

Young, J.S., Scoville, N.Z.: Annu. Rev. Astron. Astrophys. 29, 581 (1991)

Zhu, Y., Wu, H., Cao, C., Li, H.: Astrophys. J. 686, 155 (2008)

Zibetti, S., Charlot, S., Rix, H.: Mon. Not. R. Astron. Soc. 400, 1181 (2009)

Zubko, V., Dwek, E., Arendt, R.G.: Astrophys. J. Suppl. Ser. 152, 211 (2004)

This manuscript was prepared with the AAS LATEX macros v5.2. 